Prepared for the U.S. Department of Energy

under Contract DE-AC05-76RL01830

This version of the user guide has been superseded. Please go to http://volttron.readthedocs.io/en/develop/ for the most recent VOLTRON documentation.

\title{
VOLTTRON: User Guide
}
RG Lutes
JN Haack
$S$ Katipamula
KE Monson
BA Akyol
BJ Carpenter
ND Tenney

April 2014

Pacific Northwest

NATIONAL LABORATORY

Proudly Operated by Battelle Since 1965 


\title{
DISCLAIMER
}

United States Government. Neither the United States Government nor any agency thereof, nor Battelle Memorial Institute, nor any of their employees, makes any warranty, express or implied, or assumes any legal liability or responsibility for the accuracy, completeness, or usefulness of any information, apparatus, product, or process disclosed, or represents that its use would not infringe privately owned rights. Reference herein to any specific commercial product, process, or service by trade name, trademark, manufacturer, or otherwise does not necessarily constitute or imply its endorsement, recommendation, or favoring by the United States Government or any agency thereof, or Battelle Memorial Institute. The views and opinions of authors expressed herein do not necessarily state or reflect those of the United States Government or any agency thereof.

\author{
PACIFIC NORTHWEST NATIONAL LABORATORY \\ operated by \\ BATTELLE \\ for the \\ UNITED STATES DEPARTMENT OF ENERGY \\ under Contract DE-AC05-76RL01830
}

Printed in the United States of America

Available to DOE and DOE contractors from the

Office of Scientific and Technical Information,

P.O. Box 62, Oak Ridge, TN 37831-0062;

ph: (865) 576-8401, fax: (865) 576-5728

email: reports@adonis.osti.gov

Available to the public from the National Technical Information Service, U.S. Department of Commerce, 5285 Port Royal Rd., Springfield, VA 22161

ph: (800) 553-6847, fax: (703) 605-6900

email: orders@ntis.fedworld.gov

online ordering: http://www.ntis.gov/ordering.htm 
PNNL-23182

\title{
VOLTTRON: User Guide
}

\author{
RG Lutes \\ JN Haack \\ S Katipamula \\ KE Monson \\ BA Akyol \\ BJ Carpenter \\ ND Tenney
}

April 2014

Prepared for U.S. Department of Energy under Contract DE-AC05-76RL01830

Pacific Northwest National Laboratory Richland, Washington 99352 


\section{Summary}

The Department of Energy's (DOE’s) Building Technologies Office (BTO) is supporting the development of the concept of "transactional network" that supports energy, operational, and financial transactions between building systems (e.g., rooftop units -- RTUs), between building systems and the electric power grid using applications, or 'agents' that reside either on the equipment, on local building controllers or in the Cloud.

As part of this Transactional Network initiative, BTO has funded Pacific Northwest National Laboratory to develop an open source, open architecture platform that enables a variety of site/equipment specific applications to transact in a cost effective and scalable way. The goal of this initiative is to lower the cost of entry for both existing and/or new service providers because the data transport or information exchange typically required for operational and energy-related products and services will be ubiquitous and interoperable.

The transactional network platform consists of VOLTTRONTM agent execution software, a number of agents that perform a specific function (fault detection, demand response, weather service, logging service, etc.). The platform is intended to support energy, operational, and financial transactions between networked entities (equipment, organizations, buildings, grid, etc.).

This document is a user guide for the deployment of the transactional network platform and agent/application development within VOLTTRON. The intent of this user guide is to provide a description of the functionality of the transactional network platform. This document describes how to deploy the platform, including installation, use, guidance, and limitations. It also describes how additional features can be added to enhance its current functionality. 


\section{Table of Contents}

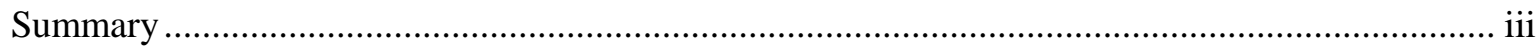

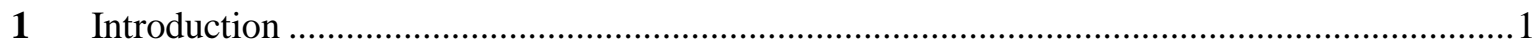

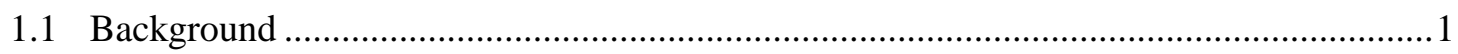

1.2 Transactional Network Platform Overview …………………………………………....

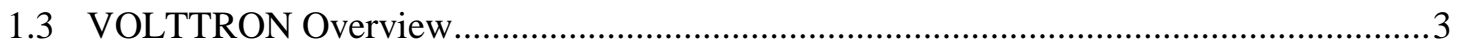

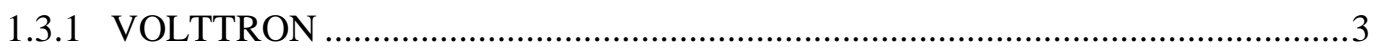

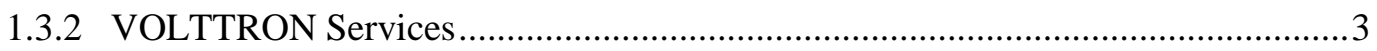

2 Deployment of VOLTTRON.................................................................................

2.1 Installing Linux Virtual Machine ................................................................................

2.2 Running and Configuring Virtual Machine......................................................................

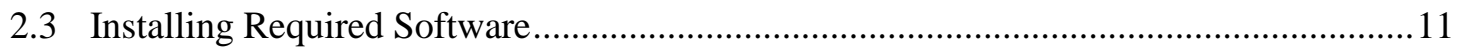

2.4 Installing the sMAP Server (Optional),.......................................................................13

2.5 Checking Out Transactional Network from Repository....................................................13

2.6 Building the VOLTTRON Platform …………………………………………….....13

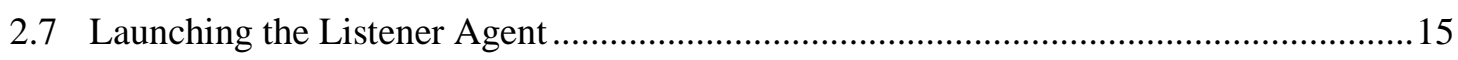

2.8 Launching the Weather Agent.......................................................................................16

2.8.1 Obtaining a Developer Key from WeatherUnderground ......................................16

2.8.2 Configuring WeatherAgent with Developer Key and Location............................18

2.8.3 Launching the Weather Agent............................................................................19

2.9 Configuring and Launching sMAP Driver ....................................................................

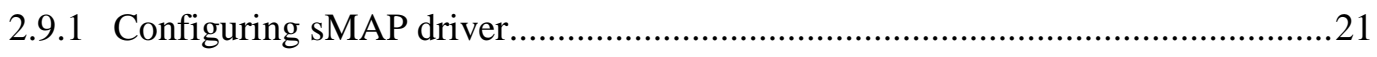

2.9.2 Launching the driver ....................................................................................24

2.10 Configuring and Launching the Actuator Agent .........................................................24

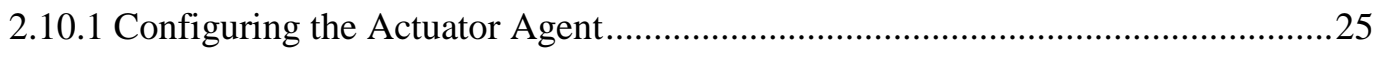

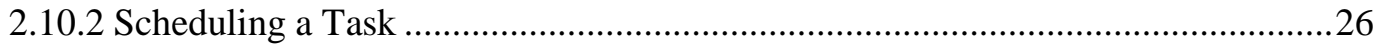

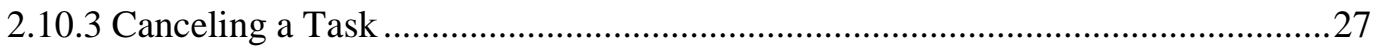

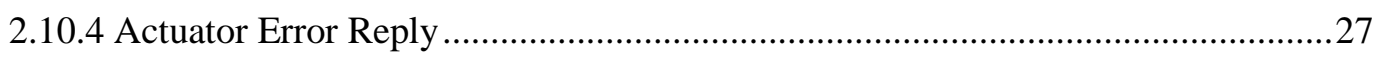

2.10.5 Task Preemption and Schedule Failure ……………….....................................28

2.10.6 Actuator Agent Interaction ..............................................................................29

2.10.7 Device Schedule State Announcements ..................................................................30

2.10.8 Launching the Actuator Agent ...........................................................................

2.10.9 Tips for Working with the Actuator Agent ..........................................................31

$3 \quad$ Sample Applications/Agents ..........................................................................................3

3.1 Automated Fault Detection and Diagnostic Agent..........................................................33

3.1.1 Configuring the AFDD agent.............................................................................33 


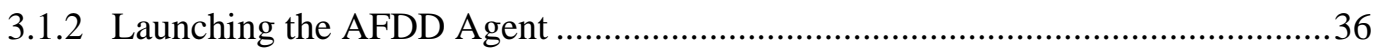

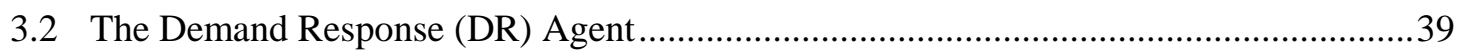

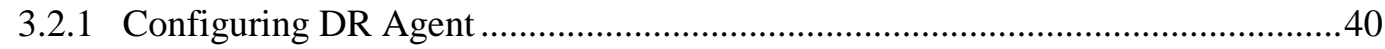

3.2.2 OpenADR (Open Automated Demand Response) ................................................4

3.2.3 DR Agent Output to sMAP ..............................................................................4

3.2.4 Launching the Demand Response Agent ............................................................4

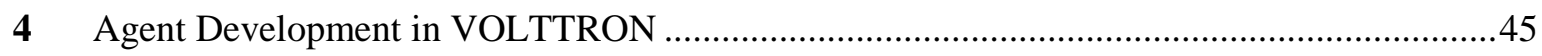

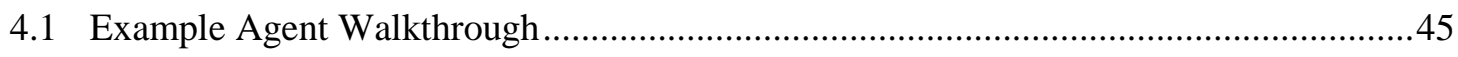

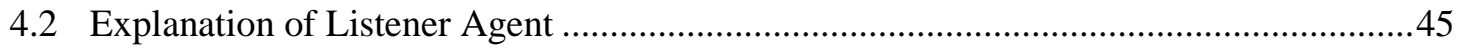

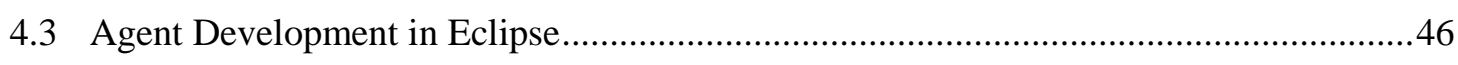

4.3.1 Setup a Run Configuration for the Platform ......................................................55

4.3.2 Setup a run configuration for the Listener Agent...................................................57

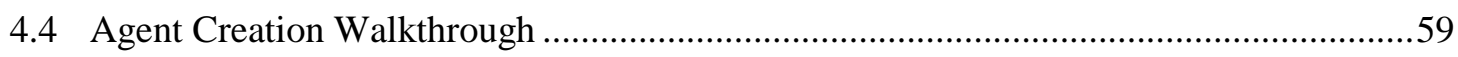

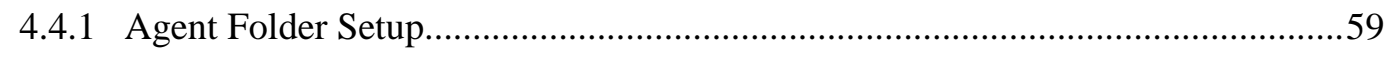

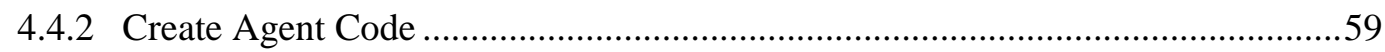

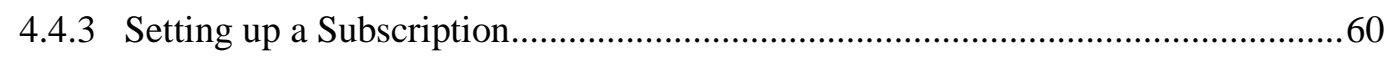

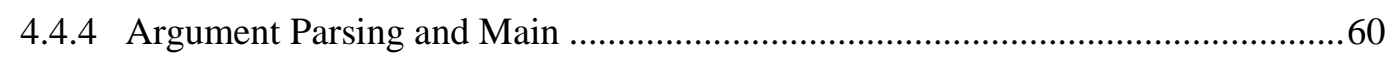

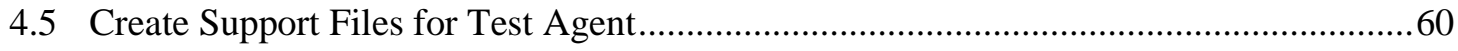

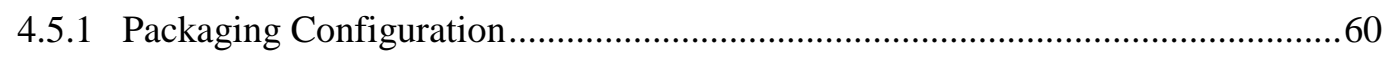

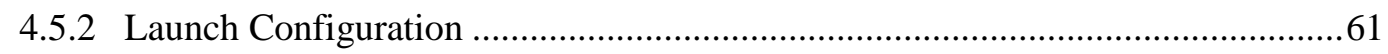

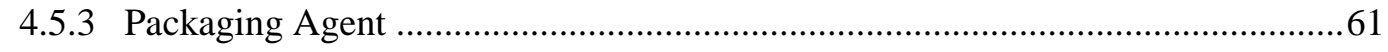

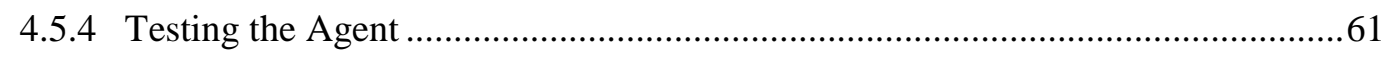

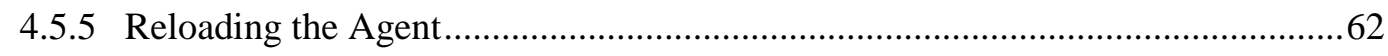

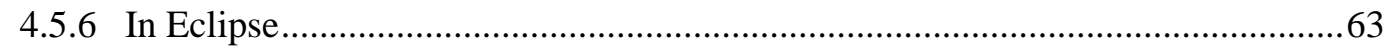

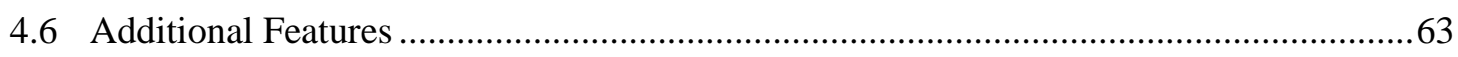

4.6.1 Subscribing to Weather Data............................................................................63

4.6.2 Utilizing the Scheduler Agent .........................................................................64

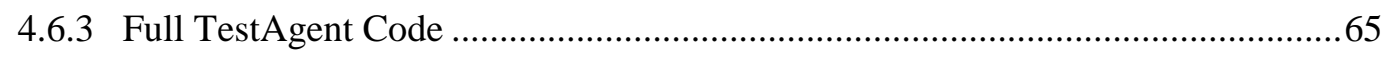

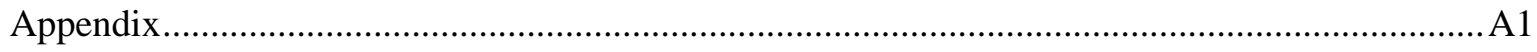




\section{Figures}

Figure 1: Illustration of the various components of the transactional network ...............................2

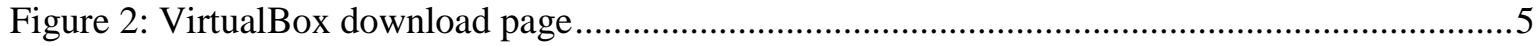

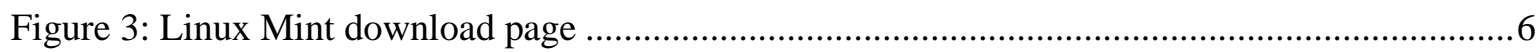

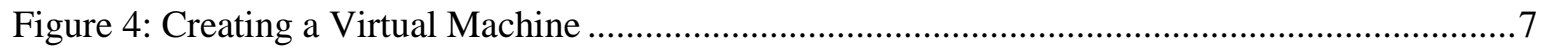

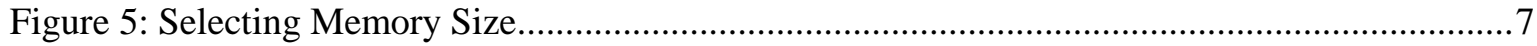

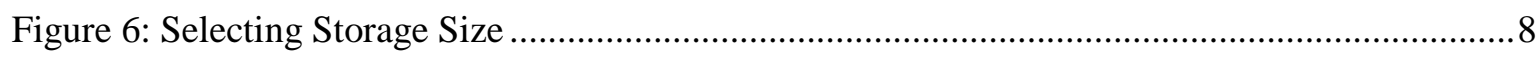

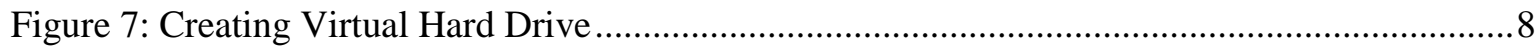

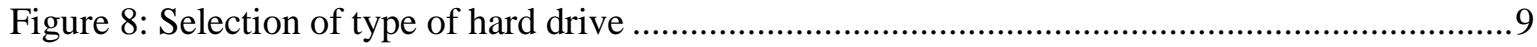

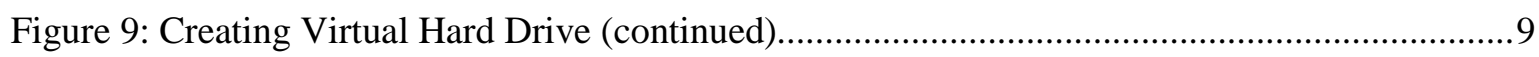

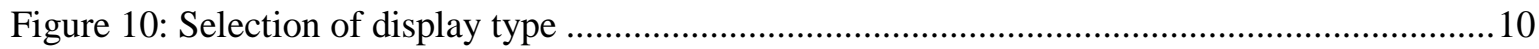

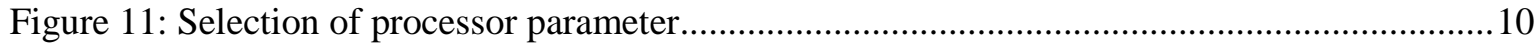

Figure 12: Loading Linux image ......................................................................................... 11

Figure 13: Installing Linux Mint Operating System ..............................................................11

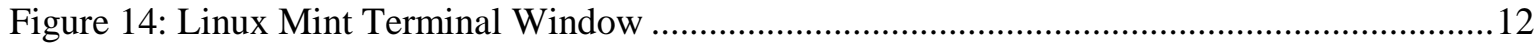

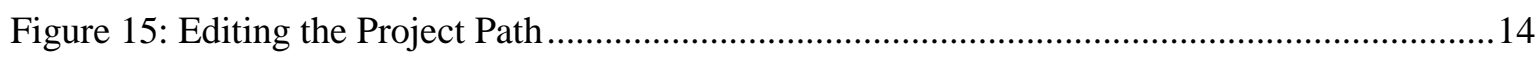

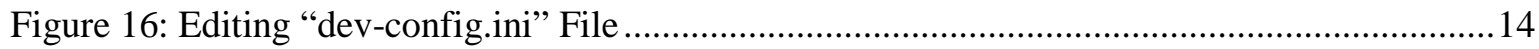

Figure 17: Sample Output from the Listener Agent....................................................................16

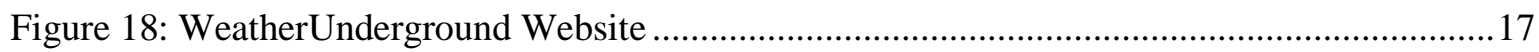

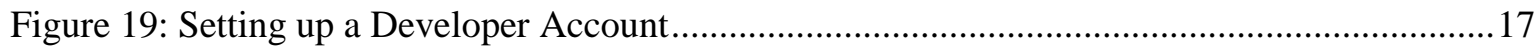

Figure 20: Creating a WeatherUnderground API Key .................................................................18

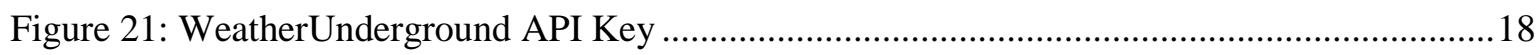

Figure 22: Entering the WeatherUnderground Developer Key .....................................................19

Figure 23: Entering Zip Code for the Location.............................................................................19

Figure 24: Example Output from the Weather Agent................................................................20

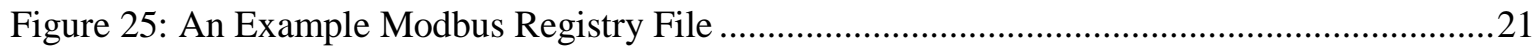

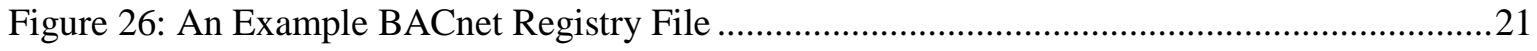

Figure 27: An Example sMAP Configuration File ....................................................................23

Figure 28: Example Actuator Agent Configuration File..............................................................25

Figure 29: Example AFDD Agent Configuration File..................................................................34

Figure 30: File Selection Dialog Box when Inputting Data in a CSV File....................................38

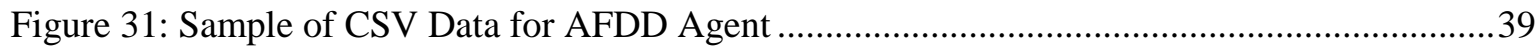

Figure 32: Example Configuration File for the DR Agent .........................................................41

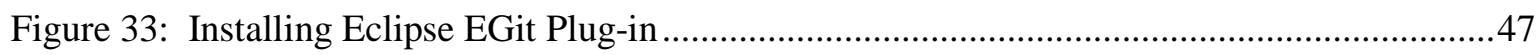

Figure 34: Installing Eclipse Egit Plug-in (continued) ............................................................. 4

Figure 35: Installing Eclipse Egit Plug-in (continued) …..........................................................48

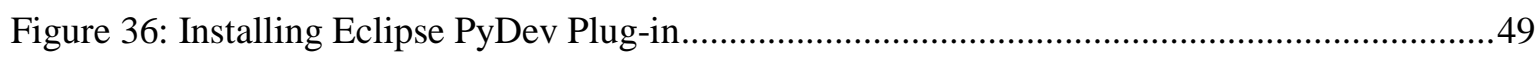

Figure 37: Checking Out Transactional Network with Eclipse ......................................................50 
Figure 38: Checking Out Transactional Network with Eclipse (continued) ..................................50

Figure 39: Checking Out Transactional Network with Eclipse (continued).................................51

Figure 40: Checking Out Transactional Network with Eclipse (continued) ...................................52

Figure 41: Checking Out Transactional Network with Eclipse (continued) ..................................52

Figure 42: Checking Out Transactional Network With Eclipse (continued) ...................................53

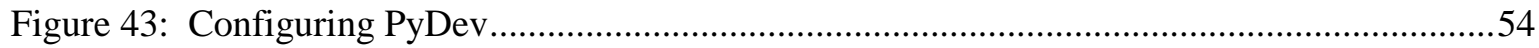

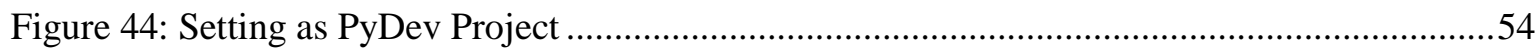

Figure 45: Setting PyDev Perspective in Eclipse.....................................................................55

Figure 46: Running Platform Configuration in Eclipse .............................................................56

Figure 47: Running Platform Configuration in Eclipse (Continued) ...........................................56

Figure 48: Running Platform Configuration in Eclipse (Continued) .............................................57

Figure 49: Configuring the ListenerAgent in Eclipse .................................................................5

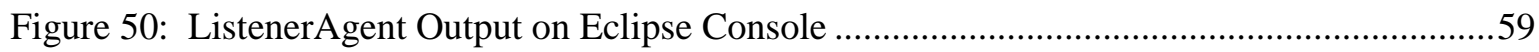

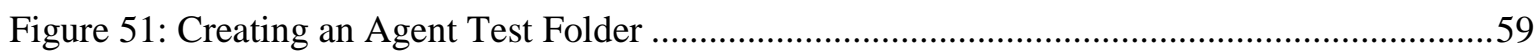

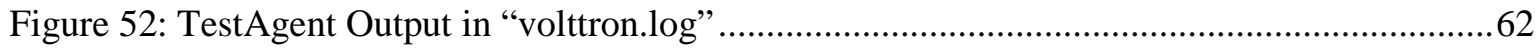

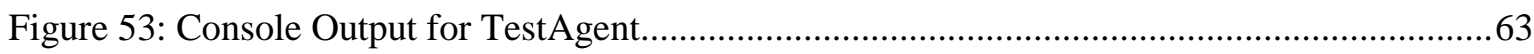

Figure 54: TestAgent Output when Subscribing to Weather Topic................................................64 


\section{Introduction}

Pacific Northwest National Laboratory (PNNL), with funding from the Department of Energy's (DOE's) Building Technologies Office (BTO), designed, prototyped and tested a transactional network platform. The platform consists of VOLTTRONTM agent execution software, a number of agents that perform a specific function (fault detection, demand response, weather service, logging service, etc.). The platform is intended to support energy, operational and financial transactions between networked entities (equipment, organizations, buildings, grid, etc.).

To encourage development and growth of the transactional network platform all the software related to VOLTTRON, platform services, and the agents within VOLTTRON are open source and employ a BSD (Berkeley Software Distribution) style license, allowing the free distribution and development of the transactional network platform. This guide is intended to give detailed instructions for the initial deployment of the transactional network platform and VOLTTRON, launch of agents (applications) on the platform, and help with development of new agents within the platform. This guide will also show how to communicate with devices (e.g., controllers, thermostats, etc.,) that utilize the Modbus or BACnet communication protocols.

\subsection{Background}

Today's building systems do not participate significantly in the energy market or provide services to power system operators. However, new smart grid technologies are creating a significant potential for building systems to participate in energy markets by providing ancillary services to power system operators. Communication networks and advanced control systems are a necessary enabler of this new potential. The transactional network platform will demonstrate the utilization of building systems (e.g., RTUs) for providing energy services to utilities using autonomous controllers. This platform will also allow for development of the next-generation control strategies and validating the strategies by:

- Quantitative analysis of energy management opportunities within buildings

- Design, prototype, and analysis of the advanced controller strategies for building systems

- Design and analysis of communication network within building and external interfaces to utility communication networks

- Economics of control strategies.

The rate and granularity of the control for the building systems determines the types of utility services that can be provided.

\subsection{Transactional Network Platform Overview}

In the transactional network platform, VOLTTRON connects devices (RTUs, building systems, meters, etc.) to applications implemented in the platform and in the Cloud, a data historian, and signals from the power grid. VOLTTRON is an agent execution platform providing services to its agents that allow them to easily communicate with physical devices and other resources. VOLTTRON also provides helper classes to ease development and deployment of agents into the environment.

Figure 1 shows the various components of the Transactional Network platform. The driver communicates to the building system controllers using Modbus or BACnet. It periodically reads data off the controller 
and both posts data to the sMAP historian and publishes data to the message bus on a topic for each device; it also provides a means to send control commands from various agents to controllers. The Actuator/Scheduler agent allows other applications on the platform to schedule times to interact with devices. This Scheduler agent ensures that multiple agents are not actively controlling a device and allows the user to set the relative priority of each application.

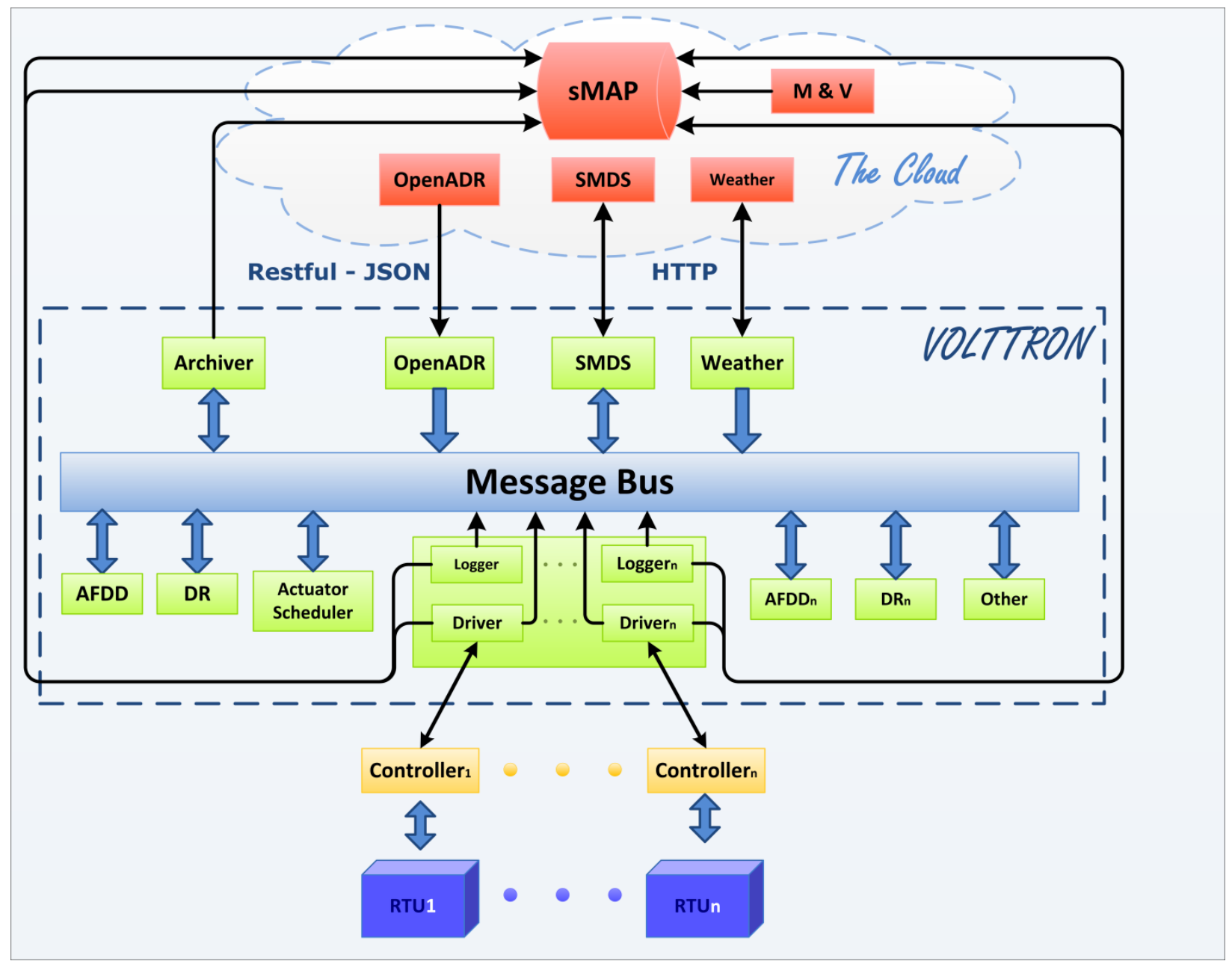

Figure 1: Illustration of the various components of the transactional network

The Archiver, in Figure 1, allows agents to request data from sMAP over the message bus. This isolates agents from the historian and allows the platform the flexibility of using potentially different data storage solutions. For example, because sMAP does not accept string data, a separate database could be used, and the interface to the agents would remain unchanged.

Agents and platform services shown in Figure 1 communicate with each other via the message bus using publish/subscribe over a variety of topics. For example, the weather agent would publish weather information to a "weather" topic that interested agents would subscribe to. The platform itself publishes platform related messages to the "platform" topic (such as "shutdown"). Topics are hierarchical following the format "topic/subtopic/subtopic", allowing agents to get as general or as specific as they want with their subscriptions. For example, agents could subscribe to "weather/all” and get all weather data for a location or "weather/temperature" for only temperature data. 


\subsection{VOLTTRON Overview}

The transactional network platform is an open-source, open-architecture platform that enables a variety of site/equipment specific applications to be applied in a cost-effective and scalable way. Such an opensource platform will lower the cost of entry for both existing and new service providers because the data transport or information exchange typically required for operational and energy related products and services would be ubiquitous and interoperable.

\subsubsection{VOLTTRON}

VOLTTRON serves as an integrating platform for the components of the transactional network. It provides an environment for agent execution and serves as a single point of contact for interfacing with devices (RTUs, building systems; meters, etc.), external resources, and platform services such as data archival and retrieval. VOLTTRON provides a collection of utility and helper classes, which simplifies agent development. VOLTTRON connects devices to applications implemented in the platform and in the Cloud, a data historian, and signals from the power grid. VOLTTRON incorporates a number of open source projects to build a flexible and powerful platform. The following is a summary of the various open source tools (software) that VOLTTRON utilizes:

- sMAP: VOLTTRON utilizes SMAP $^{1}$ for data storage and retrieval. The VOLTTRON Modbus driver publishes data from devices to the platform and also stores the data in the sMAP historian. During development of this driver, the VOLTTRON team contributed error reports and resolved a bug in the sMAP software.

- Drivers for sMAP are written using another open source product called twistd ${ }^{2}$. Twistd is an event-based networking engine.

- OMQ: The VOLTTRON message bus, which allows agents and services to exchange data, uses Zero $\mathrm{MQ}^{3}$. This free software is used by National Aeronautics and Space Administration (NASA), Cisco, etc. to provide scalable, reliable, and fast communication. The VOLTTRON team has reported security and bug fixes as well as changes to the software to correct the bugs were contributed back.

- PyModbus: The VOLTTRON Modbus ${ }^{4}$ driver builds on PyModbus ${ }^{5}$, which enables Python code to easily interact with Modbus devices.

- Other open source Python modules being used are:

o 'avro', 'configobj', 'gevent', 'flexible-jsonrpc', 'numpy', 'posix-clock', 'pyopenssl', 'python-dateutil', 'requests', 'setuptools', 'simplejson', 'zope.interface'

\subsubsection{VOLTTRON Services}

VOLTTRON's services utilize the above mentioned open source tools in conjunction with other applications developed by collaborators, these services/applications include:

\footnotetext{
${ }^{1}$ http://www.cs.berkeley.edu/ stevedh/smap2/index.html

${ }^{2}$ http://twistedmatrix.com/trac/

${ }^{3}$ http://zeromq.org/

${ }^{4}$ http://www.modbus.org/

${ }^{5}$ http://code.google.com/p/pymodbus/
} 
- Actuator Agent: This platform service is deployed in the form of an agent running on VOLTTRON. The Actuator agent manages the control of external devices (e.g., RTUs) by agents within VOLTTRON.

o Device control: The Actuator agent will accept commands from other agents and issue the commands to the specified device. Currently MODBUS and BACnet compatible device communication is supported.

o Device access scheduling: This service allows the scheduling of agents' access to devices to prevent multiple agents from controlling the same device at the same time.

- Message Bus: All agents and services can publish and subscribe to topics on the message bus. This provides a single and uniform interface that abstracts the details of devices and agents from each other. At the most basic level, agents and components running in the platform produce and consume messages, events. The details of how agents produce events and how they process received events are left up to the agents.

- Weather Information: This platform service is deployed in the form of an agent running on VOLTTRON. This agent periodically retrieves data from the Weather Underground site. It then reformats the data and publishes it out to the platform on a weather topic accessible to other agents.

- Logging Service: Agents can publish integer or double data to arbitrary paths to a logging topic and this service will push them to the sMAP historian for later analysis. The primary use of the Logging Service is to allow agents to record actions or results from the agent executing its services.

Agents deployed on VOLTTRON can perform one or more roles, which can be broadly classified into the following groups:

- Cloud Agents: These agents are part of a remote application that needs access to the messages and data on the platform. A Cloud agent would subscribe to topics of interest to the remote (or Cloud) application and would allow it to publish data from remote application to the platform.

- Control Agents: These agents control the devices of interest and interact with other resources to achieve a goal.

- Passive Agent: These agents subscribe to certain data from the building systems and perform certain actions to create knowledge (faulty operation). The information and knowledge that these agents create is posted to the historian or in a local file. 


\section{Deployment of VOLTTRON}

VOLTTRON has been developed for deployment on Linux operating systems. To use VOLTTRON on a Mac or Windows system VOLTTRON must be deployed on a virtual machine (VM). A VM is a software implementation of a machine (i.e., a computer) that executes programs like a physical machine. A system VM provides a complete system platform, which supports the execution of a complete operating system (OS). These usually emulate an existing architecture, and are built with the purpose of providing a platform to run programs where the real hardware is not available for use. This document will describe the steps necessary to install VOLTTRON on a Windows system using Oracle VirtualBox software (Figure 2).

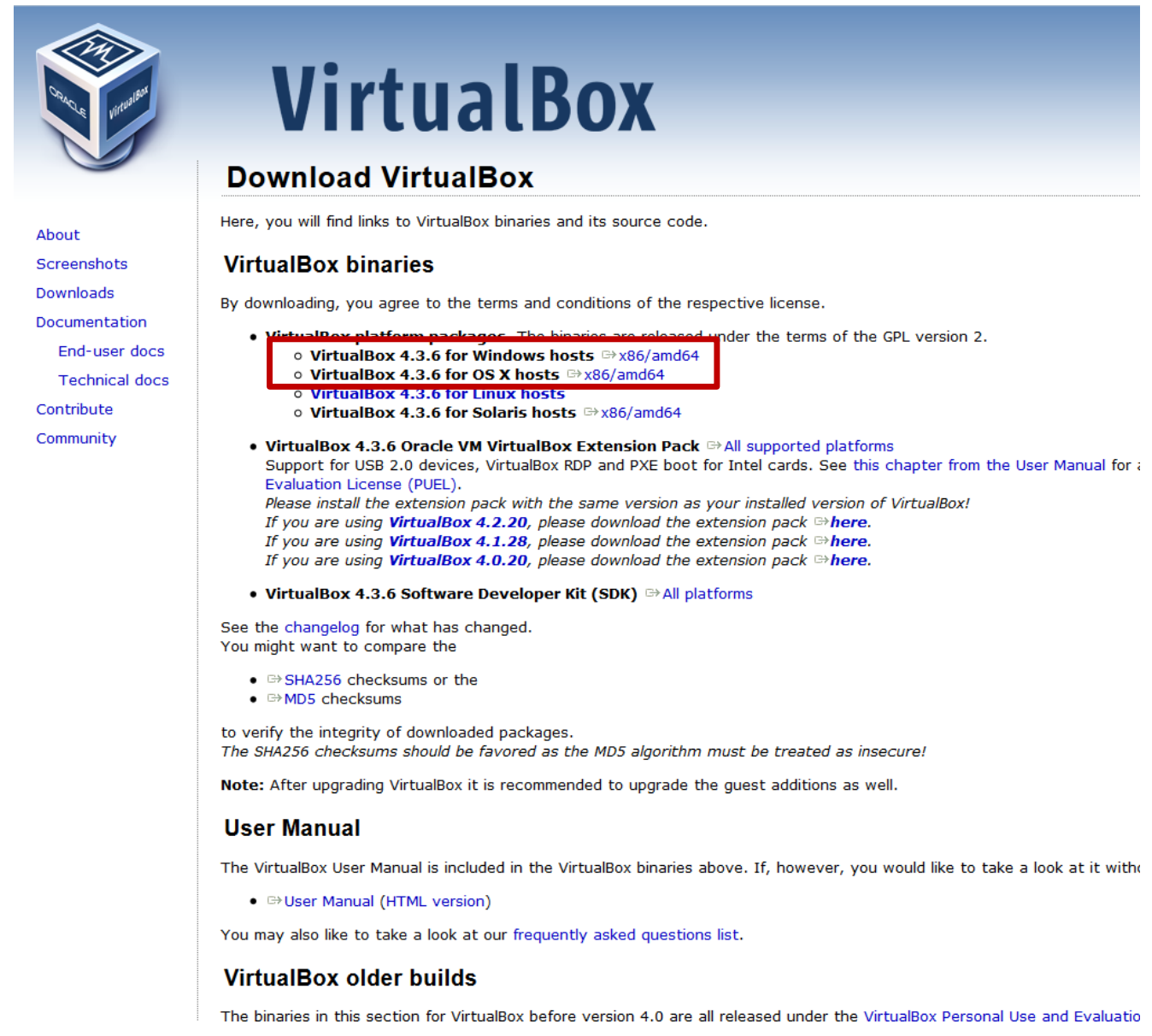

Figure 2: VirtualBox download page

\subsection{Installing Linux Virtual Machine}

VirtualBox is free and can be downloaded from https://www.virtualbox.org/wiki/Downloads. Figure 2 shows the VirtualBox download page. The Windows and Mac host OS is shown boxed in red in the figure.

To install on Windows choose: VirtualBox for Windows hosts x86/amd64 
To install on Mac choose: VirtualBox for OS X hosts x86/amd64

The latest version of VirtualBox, when this guide was constructed, was VirtualBox 4.3.6. VOLTTRON should be compatible with future releases of VirtualBox. After the installation file is downloaded, run and install the VirtualBox software. It will also be necessary to download a Linux operating system image for use on the VM. Ubuntu 12.04 LTS or Linux Mint is the recommended Linux operating system for use with VOLTTRON. This document will describe the use of VOLTTRON with Linux Mint. Any distribution of Linux Mint or Ubuntu should work with VOLTTRON but this document will describe the development of agents within VOLTTRON where the Xfce desktop is used. The other desktops associated with Linux Mint are compatible with VOLTTRON and should provide similar functionality to the Xfce desktop (Figure 3). Linux Mint can be downloaded from the following

URL http://www.linuxmint.com/download.php. Set up of the platform in Ubuntu is identical to the set up in Linux Mint ${ }^{6}$ except for changes in the appearance of the desktop. If running VOLTTRON on a system with limited hardware, less than 2 GB of RAM, a 32-bit version of Linux should be used.

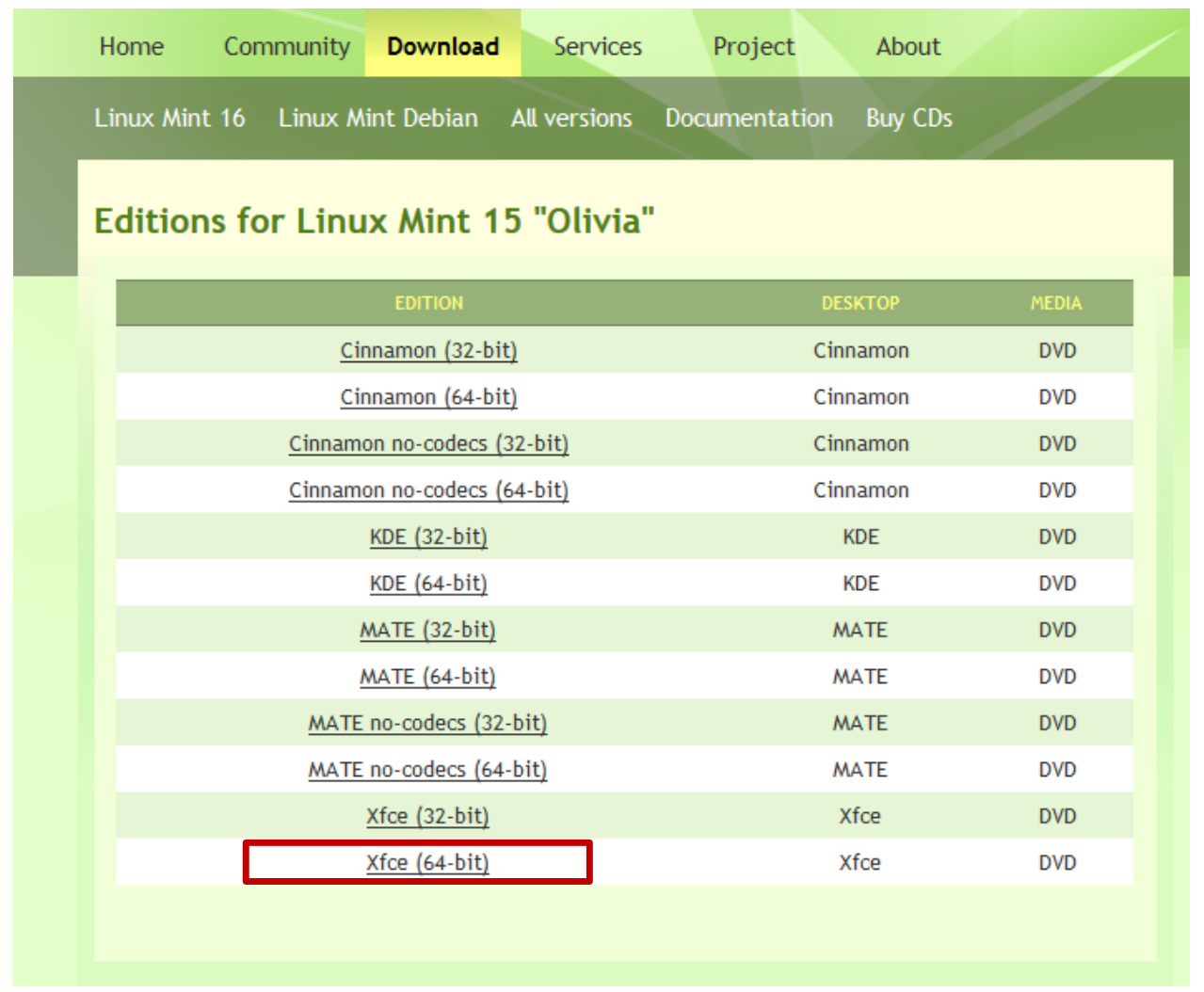

Figure 3: Linux Mint download page

\subsection{Running and Configuring Virtual Machine}

After the VirtualBox software is installed and the Linux Mint image has been downloaded, the virtual machine can be run and configured. The following steps will describe how to configure the VM for deployment of VOLTTRON:

\footnotetext{
${ }^{6}$ Note that Linux Mint version could be different from the shown here. Also, on the download screen, you could pick any site, but preferably the site that is close to you.
} 
1. Start VirtualBox and click "New" icon in the top left corner of Oracle VM VirtualBox Manager Window.

2. A selection box will appear; configure the selection as shown in Figure 4. Choose Next.

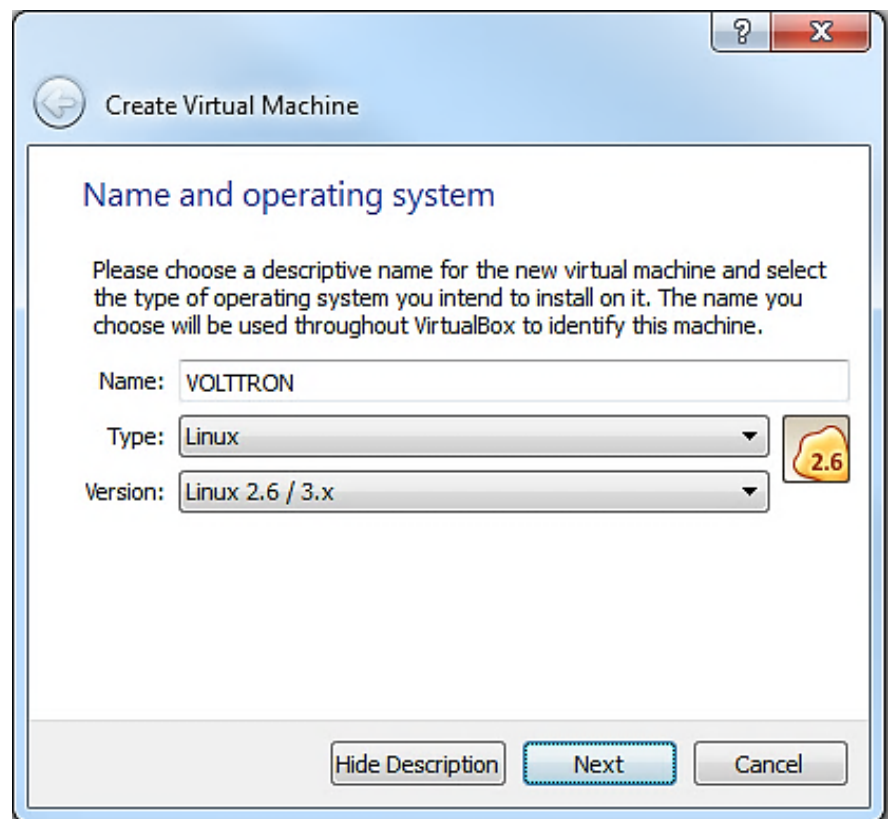

Figure 4: Creating a Virtual Machine

3. Choose the amount of memory to allot the VM as shown in following figure. Note that this memory will be unavailable to the host while running the VM (i.e., a computer with 4 GB of memory, could probably spare 1 GB for the VM). Choose Next.

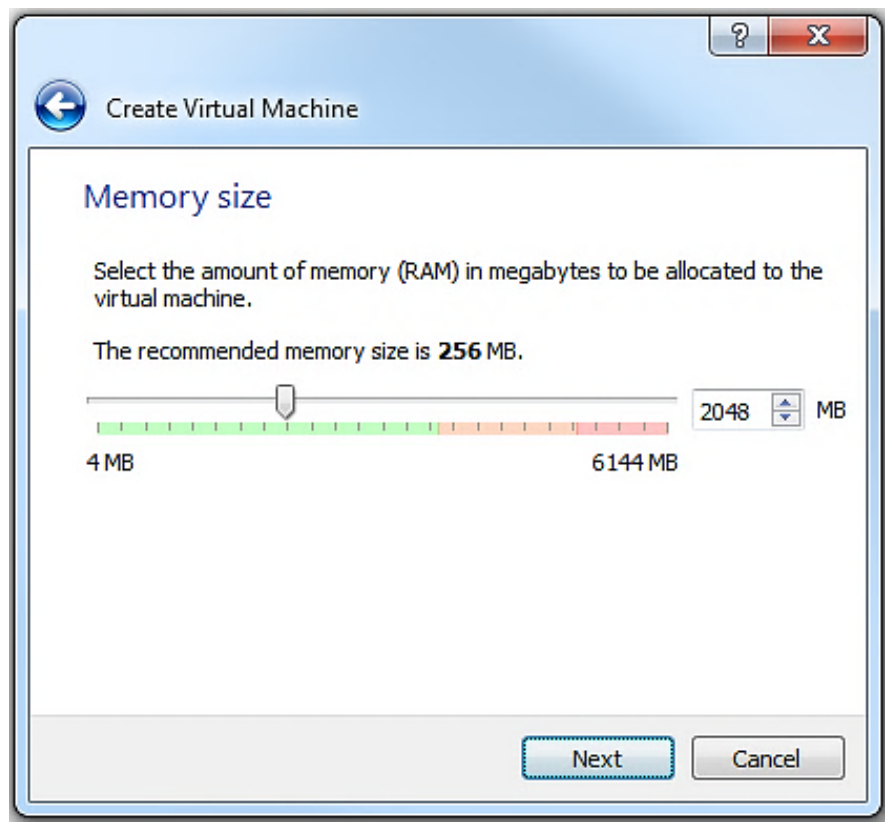

Figure 5: Selecting Memory Size 
4. Create hard drive for VM. Choose Create.

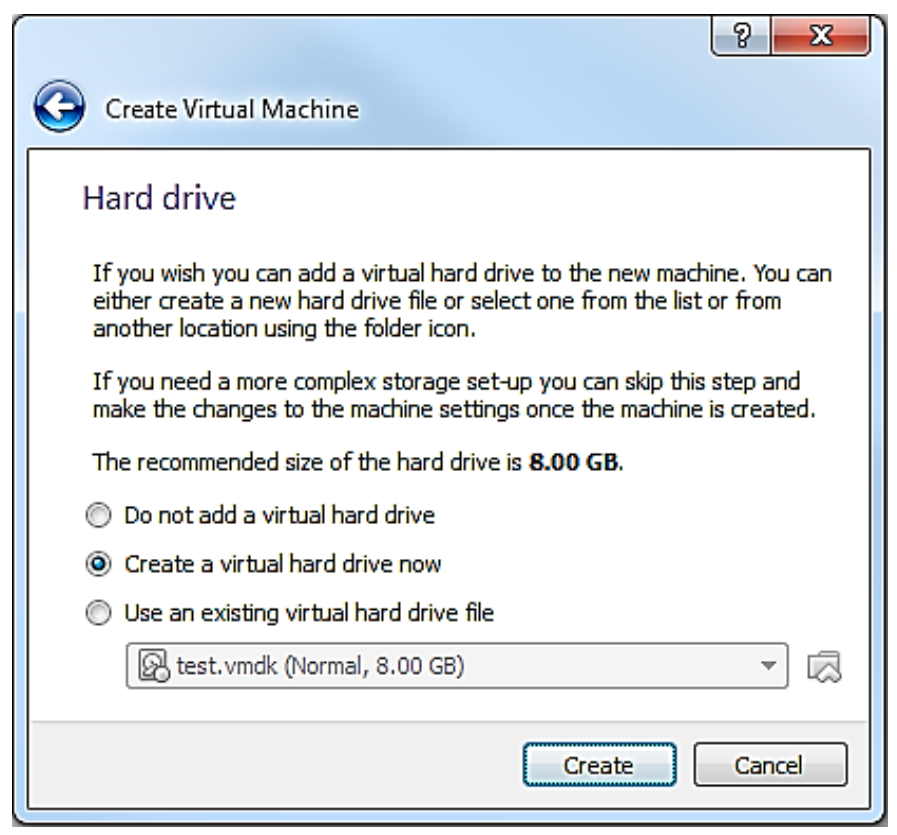

Figure 6: Selecting Storage Size

5. Choose disk type. As shown in the Figure 7, select VMDK and then select Next.

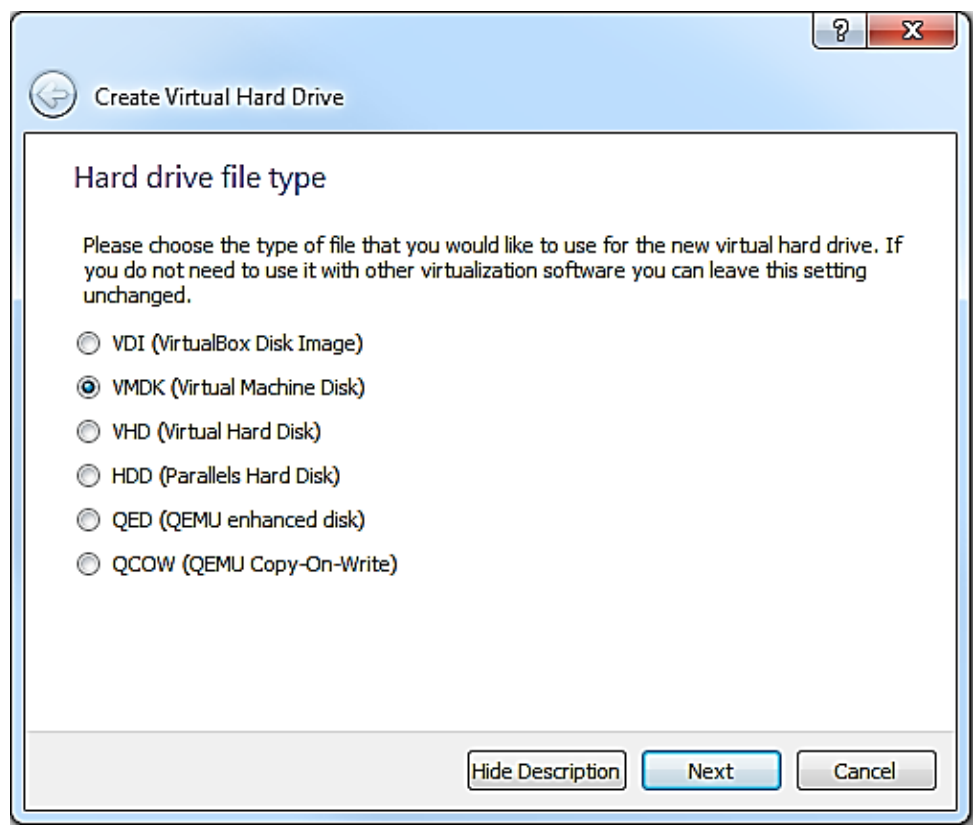

Figure 7: Creating Virtual Hard Drive

6. Choose Dynamically Allocated for the VM hard drive (Figure 8). This will allow the VM hard drive to only take storage space as is needed up to the size limit chosen in the previous step. Choose Continue. 


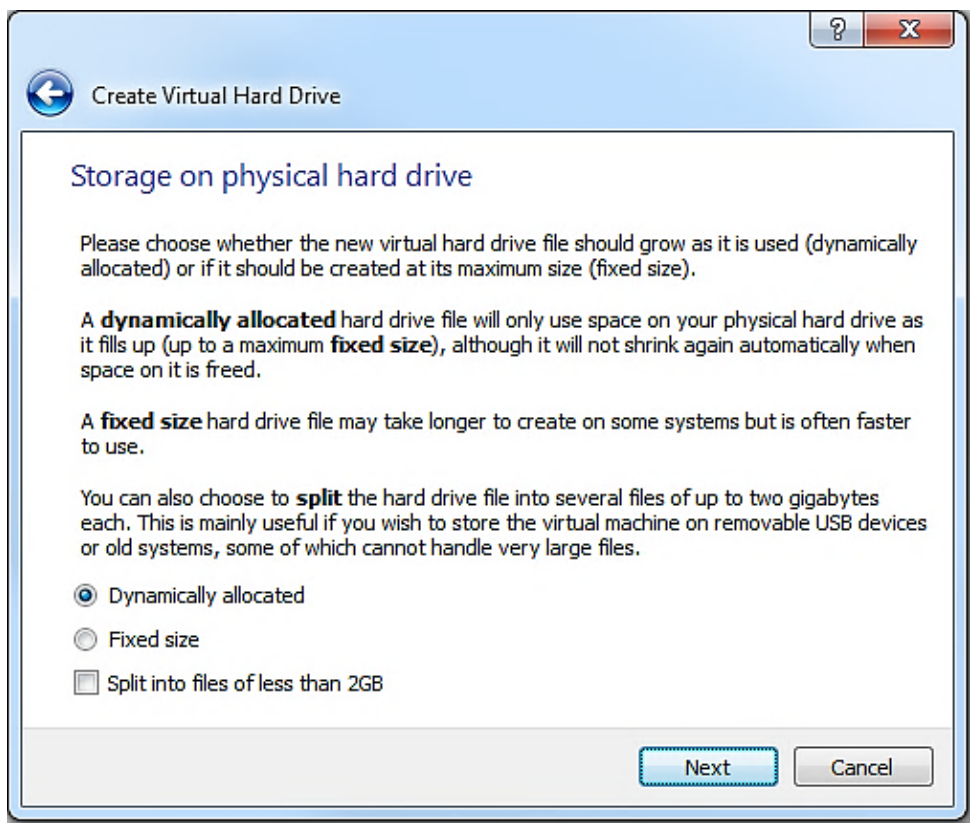

Figure 8: Selection of type of hard drive

7. Choose the file size for the VM virtual hard drive. Keep in mind that Linux is over $1 \mathrm{~GB}$ just for the operating system (Figure 9). Choose Create.

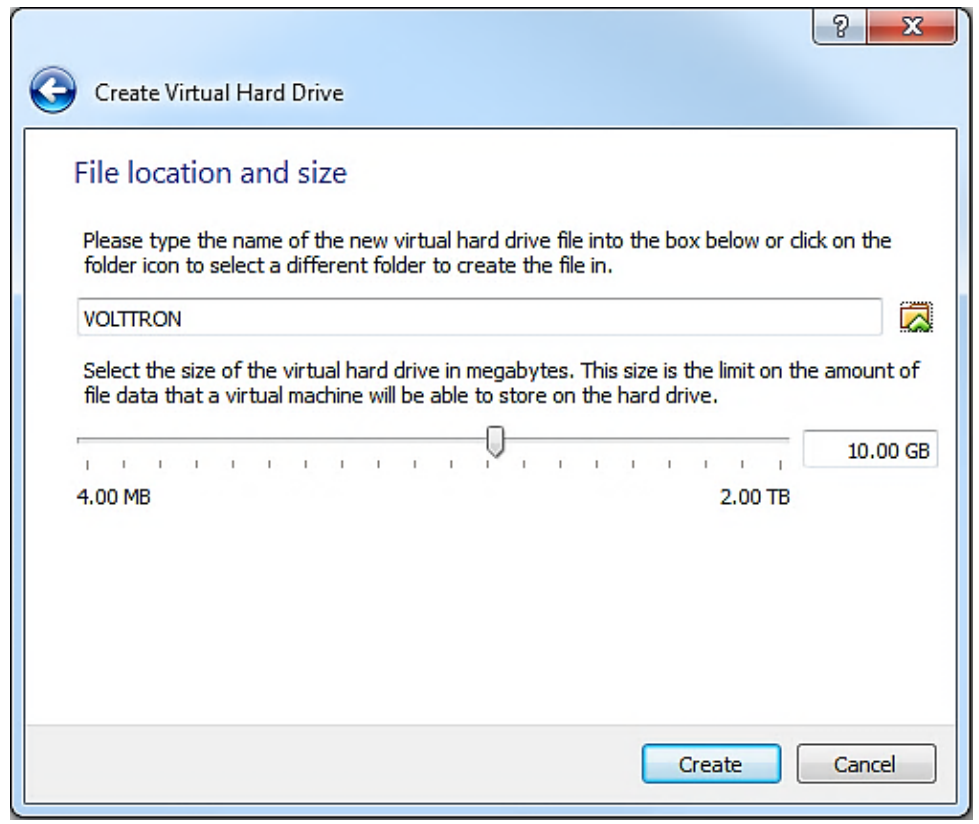

Figure 9: Creating Virtual Hard Drive (continued)

8. With the newly created VM selected choose Machine from the VirtualBox menu in the top left corner of the VirtualBox window; from the drop down menu choose Settings. In the Display menu check Enable 3D Acceleration (Figure 10). Choose OK. 


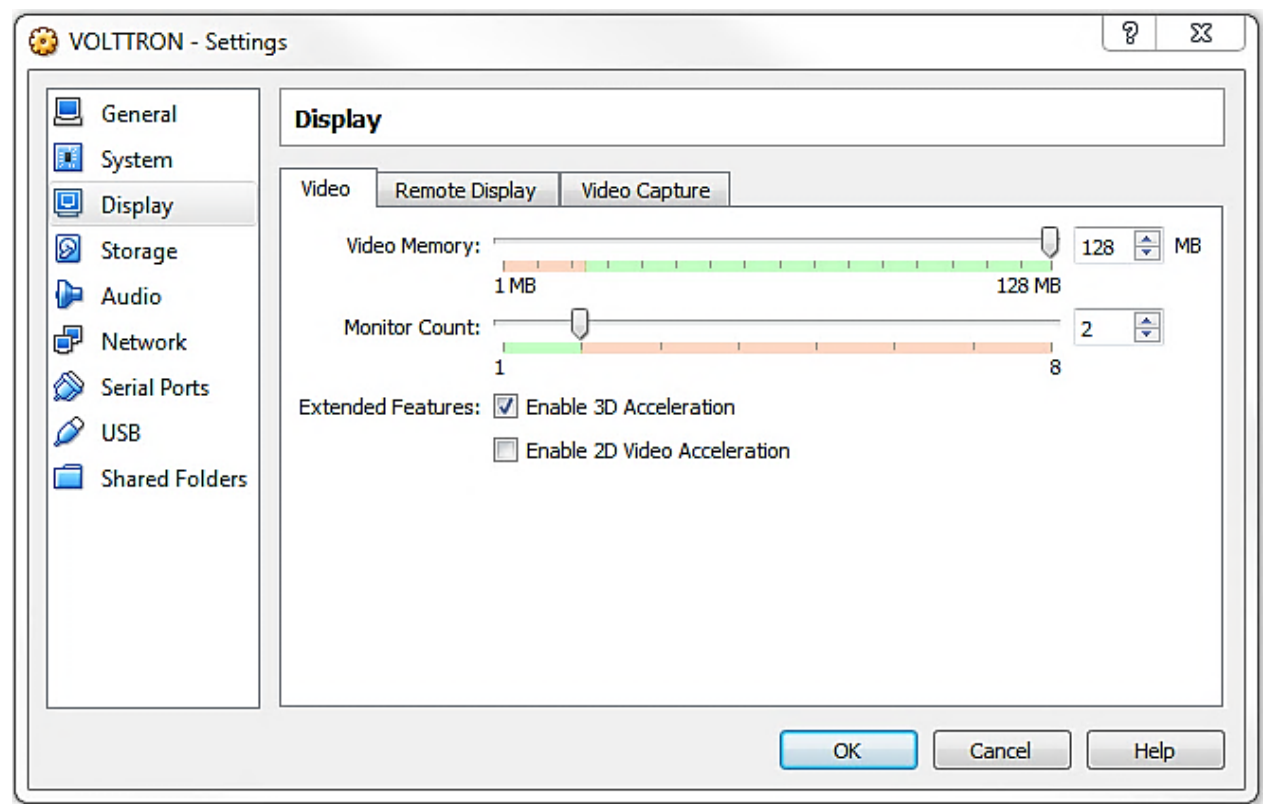

Figure 10: Selection of display type

9. With the newly created VM selected choose Machine from the VirtualBox menu in the top left corner of the VirtualBox window; from the drop down menu choose Settings. In the system menu go to the Processor tab and Enable PAE/NX (Figure 10). Choose OK.

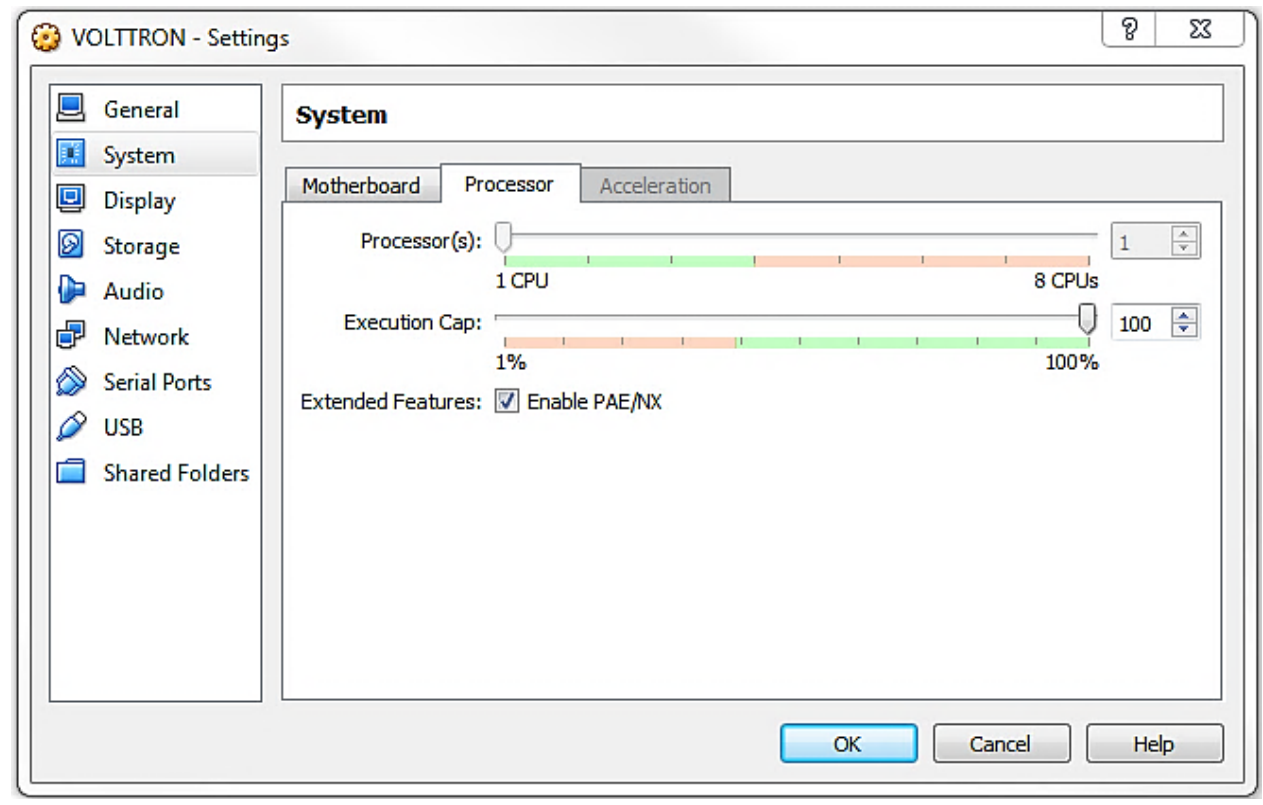

Figure 11: Selection of processor parameter

10. With the newly created VM selected click start (or right click the VM and choose start). To load the Linux image, select the Linux Mint image file (iso file) you downloaded, then choose Start. 


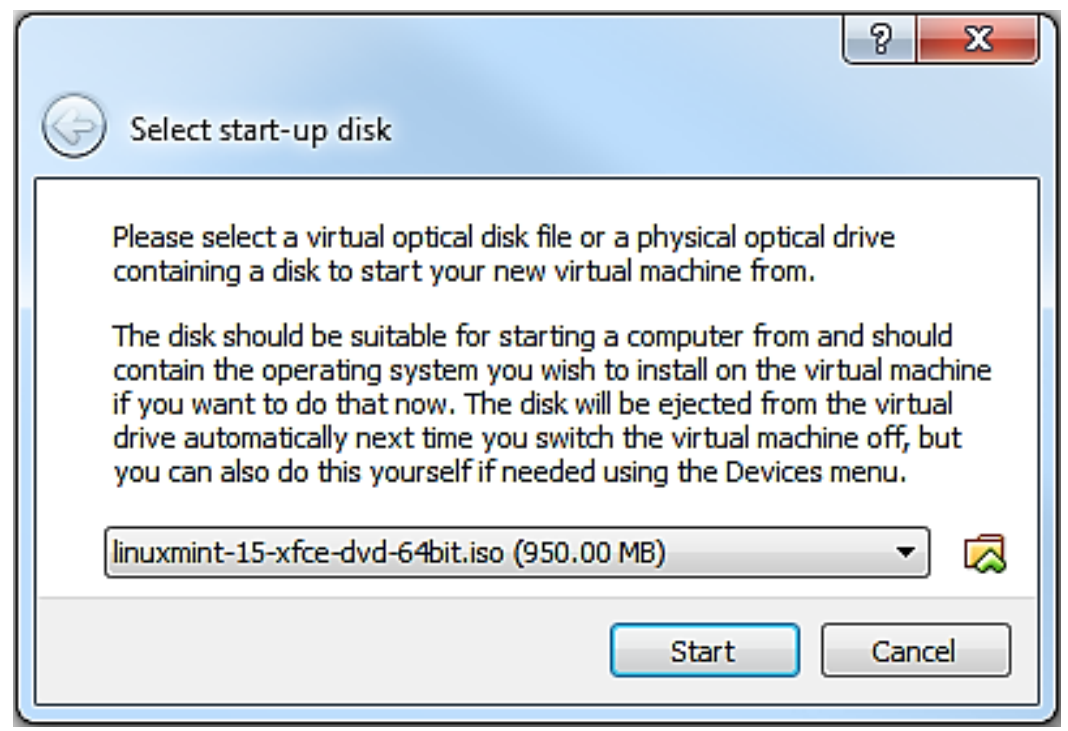

Figure 12: Loading Linux image

11. Choose Install Linux Mint (the install icon looks like a DVD media, as shown in Figure 13), proceed to configure installation (language, etc.). The VM will now have Linux Mint installed permanently.

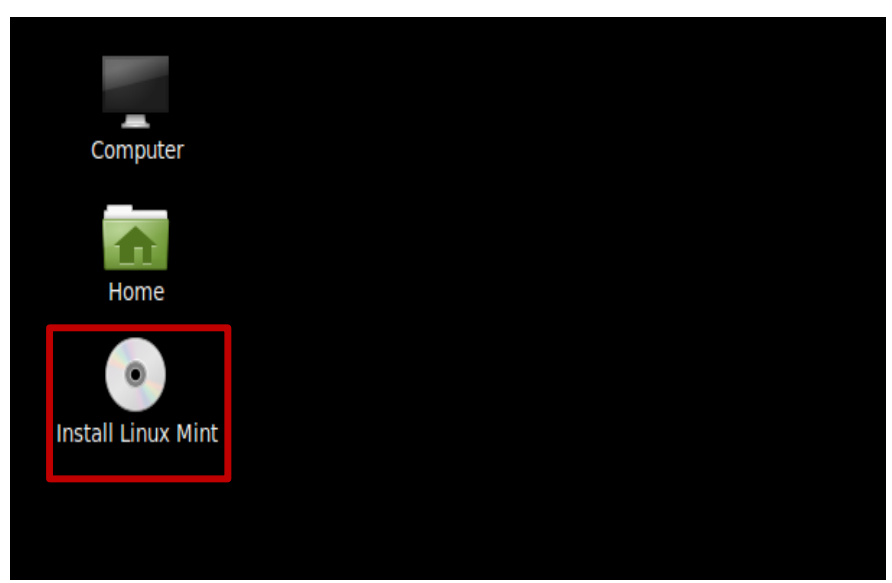

Figure 13: Installing Linux Mint Operating System

\subsection{Installing Required Software}

VOLTTRON requires the following Linux modules. To install them, open a terminal window and enter the following commands (terminal commands are bold): 


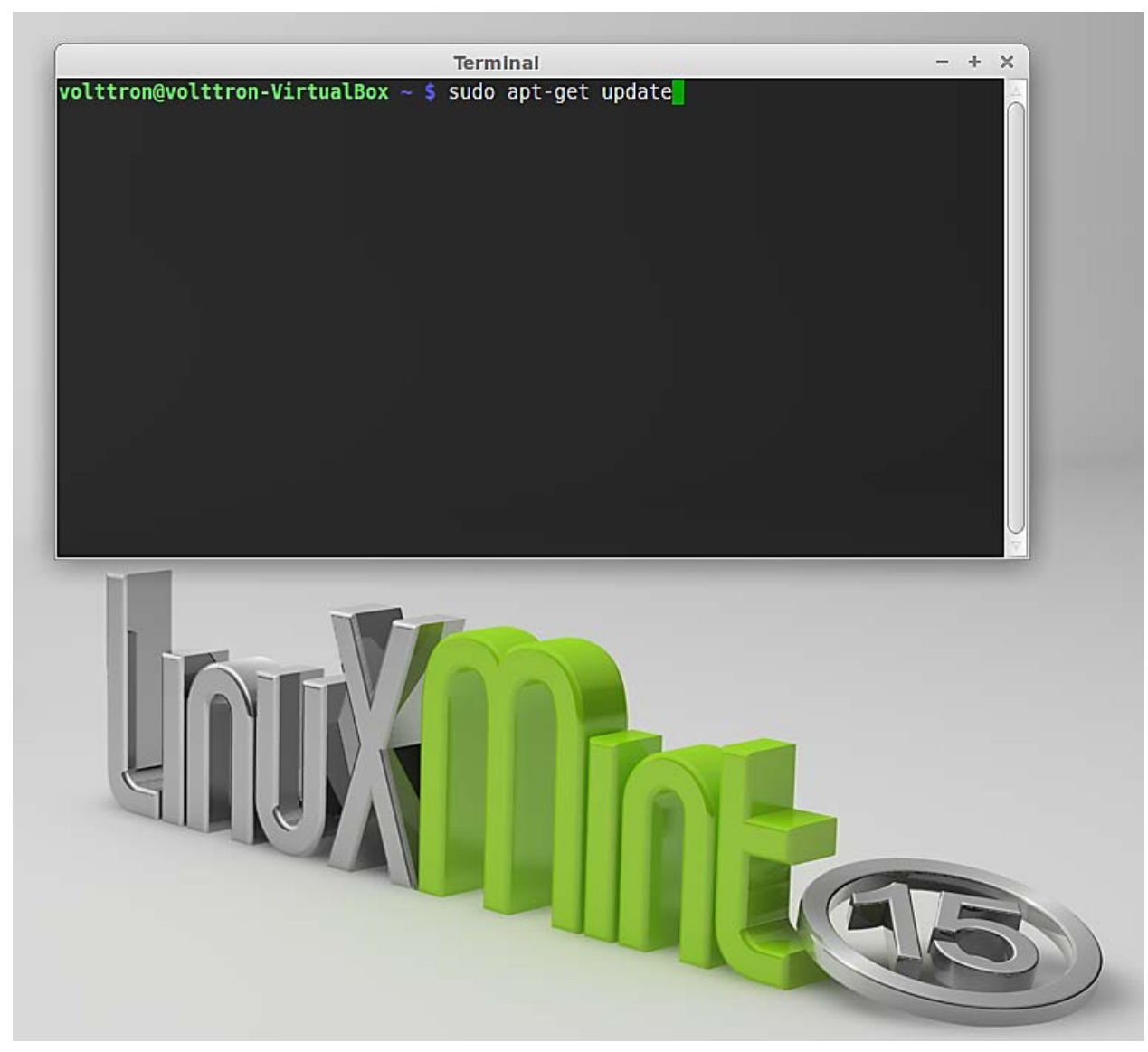

Figure 14: Linux Mint Terminal Window

- $\quad$ Ensures the installer is up to date:

o sudo apt-get update

- This installs Git. The transactional network source code including VOLTTRON and other agent code is stored in a Git repository:

0 sudo apt-get install git

- This installs Python DevTools. This is a Python software development tool necessary for running VOLTTRON:

o sudo apt-get install python-dev

- $\mathrm{g}++$ is a $\mathrm{C}++$ compatible runtime library:

o sudo apt-get install $\mathrm{g}^{++}$

- Required development library:

o sudo apt-get install libevent-dev

0 sudo apt-get install libssl-dev

- Required Python module:

0 sudo apt-get install python-tk 


\subsection{Installing the sMAP Server (Optional)}

VOLTTRON uses SMAP as its data repository for storing data from devices and log messages from agents. If you have access to an existing sMAP server, you can configure your VOLTTRON instance to work with that as in 2.9.1.

To install your own sMAP instance, follow the installation instructions from the following URL: http://pythonhosted.org/Smap/install.html

We recommend skipping "Installing from Source" and installing it on the recommended OS.

\subsection{Checking Out Transactional Network from Repository}

Ensure you have installed the required packages before proceeding. We recommend creating a directory, /workspace, some of the source code for VOLTTRON is configured assuming this file structure. Enter the following commands (terminal commands are bold).

1. Creates the workspace directory:

- mkdir workspace

2. Go to workspace directory:

- cd workspace

3. Downloads the transactional network source code and creates a local copy on your machine:

- git clone https://github.com/VOLTTRON/volttron

\subsection{Building the VOLTTRON Platform}

In the workspace directory, enter the following commands (terminal commands are bold and any explanation is contained within parenthesis):

4. Go to volttron directory:

\section{- cd volttron}

5. VOLTTRON includes scripts that automatically pull down dependencies and build the necessary libraries. The "bootstrap" script has to be run only once. The bootstrap process can take up to 20 minutes; allow the script to complete before proceeding:

\section{- ./bootstrap}

If the bootstrap fails before finishing (for instance from a timeout), run:

\section{- $\quad$ bin/buildout -N}

- If bootstrap or buildout fails, try "bin/buildout -N" again.

Also, some packages (especially numpy) can be very verbose when they install. Please wait for the wall of text to finish and do not be alarmed by any warnings. If the script finishes without any exception, the bootstrap was successful. 
6. Edit the dev-config file to ensure the paths match your installation:

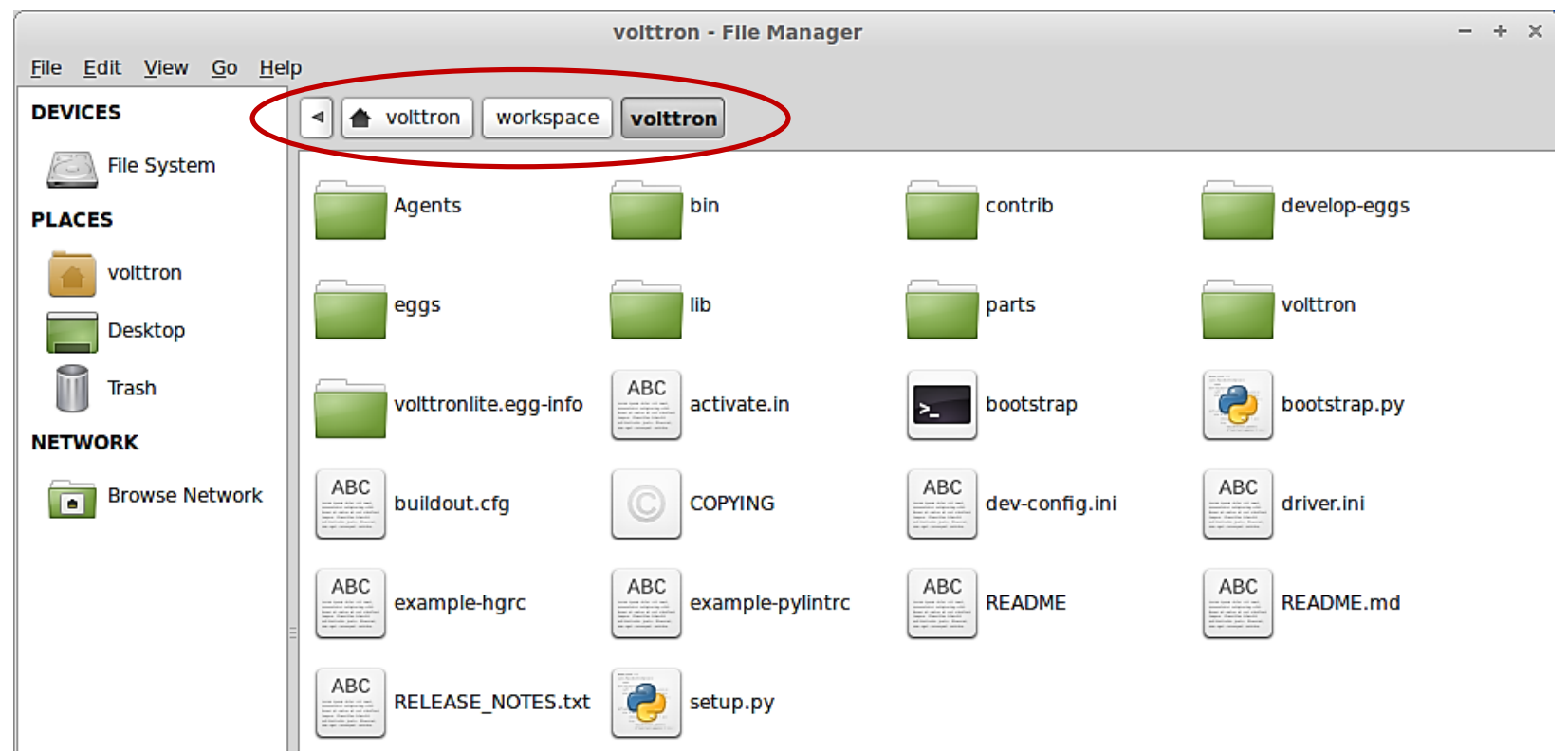

This is the file path to volttron project. Edit the dev-config.ini to match. The " " denotes the path to the users home directory, in this case: "/home/volttron."

Figure 15: Editing the Project Path

dev-config.InI ( /workspace/volttron) - gedit

File Edit View Search Iools Documents Help

†它、㖓昌|

国 dev-config.ini $*$

[ [agent-exchange]

append-pid $=$ false

[agent-paths ]

agents-dir $=\sim /$ workspace/volttron/Agents

autostart-dir $=\sim /$ workspace/volttron/autostart

bin-dir $=\sim /$ workspace/volttron $/$ bin

run-dir $=/$ tmp

This is the contents of the dev-config.ini file. It can opened with any text editor or with the Linux command nano in a terminal window.

Figure 16: Editing "dev-config.ini" File 
7. Starts VOLTTRON and logs platform activity in voltron.log (text file):

- bin/volttron-lite -c dev-config.ini -l volttron.log -v -v \&

At this point, all required software has been installed and basic configuration has been completed. Next, the installation has to be tested.

\subsection{Launching the Listener Agent}

To test the VOLTTRON installation, build and deploy the Listener agent. If one plans on utilizing an integrated development environment (IDE) for agent development please refer to section 4.3 for information on installing and running agents in the Eclipse IDE. The Listener agent is a VOLTTRON platform agent. The Listener agent logs all activity on the message bus for a particular instance of VOLTTRON. This agent can be helpful when debugging an application or for monitoring what is being published on the message bus by other agents.

From the volttron directory enter the following commands in a terminal window:

1. Build the Listener agent eggsecutable:

- $\quad$ volttron/scripts/build-agent.sh ListenerAgent

2. Change permissions on the eggsecutable file:

- $\quad$ chmod +x Agents/listeneragent-0.1-py2.7.egg

3. Install the eggsecutable:

- bin/volttron-ctrl install-executable Agents/listeneragent-0.1-py2.7.egg

4. Load the agent's configuration file:

- bin/volttron-ctrl load-agent Agents/ListenerAgent/listeneragent.launch.json

5. Start the agent:

\section{- bin/volttron-ctrl start-agent listeneragent.launch.json}

6. Show the Listener agent's output, from the volttron.log:

- tail volttron.log

If changes are made to the Listener agent's configuration file after the agent is launched it is necessary to stop and reload the agent. See section 4.5.5 on instructions for reloading an agent. 


\section{Terminal}

2013-11-26 09:51:17,189 (listeneragent.launch.json 1879) listener.agent DEBUG: T

opic: heartbeat/listeneragent, Headers: Headers(\{'Date': '2013-11-26 17:51:17.18

7428Z', 'AgentID': 'Listener1', 'Content-Type': 'text/plain'\}), Message: ['2013-

11-26 17:51:17.187428Z']

2013-11-26 09:51:22,190 ( listeneragent. launch.json 1879) listener.agent DEBUG: T

opic: heartbeat/listeneragent, Headers: Headers (\{'Date': '2013-11-26 17:51:22.18

8462Z', 'AgentID': 'Listener1', 'Content-Type': 'text/plain'\}), Message: [ '2013-

11-26 17:51:22.188462Z']

2013-11-26 09:51:27,188 (listeneragent. launch.json 1879) listener.agent DEBUG: T

opic: heartbeat/listeneragent, Headers: Headers ( \{'Date': '2013-11-26 17:51:27.18

6290Z', 'AgentID': 'listener1', 'Content-Type': 'text/plain'\}), Message: [ '2013-

$\left.11-2617: 51: 27.186290 Z^{\prime}\right]$

2013-11-26 09:51:32,189 (listeneragent.launch.json 1879) listener.agent DEBUG: T

opic: heartbeat/listeneragent, Headers: Headers (\{'Date': '2013-11-26 17:51:32.18

8072Z', 'AgentID': 'Listenerl', 'Content-Type': 'text/plain'\}), Message: ['2013-

11-26 17:51:32.188072Z']

2013-11-26 09:51:37,187 (listeneragent.launch.json 1879) listener.agent DEBUG: T

opic: heartbeat/listeneragent, Headers: Headers (\{'Date': '2013-11-26 17:51:37.18

6259Z', 'AgentID': 'listener1', 'Content-Type': 'text/plain'\}), Message: ['2013-

11-26 17:51:37.186259Z']

2013-11-26 09:51:42,188 (listeneragent.launch.json 1879) listener.agent DEBUG: T

opic: heartbeat/listeneragent, Headers: Headers(\{'Date': '2013-11-26 17:51:42.18

6993Z', 'AgentID': 'listener1', 'Content-Type': 'text/plain'\}), Message: ['2013-

11-26 17:51:42.186993Z'

Figure 17: Sample Output from the Listener Agent

\subsection{Launching the Weather Agent}

The Weather agent, another VOLTTRON service agent, retrieves weather information from the WeatherUnderground site and shares it with agents running on the platform. The first step to launching the Weather agent is to obtain a developer key from WeatherUnderground.

\subsubsection{Obtaining a Developer Key from WeatherUnderground}

Follow these steps to create a WeatherUnderground account and obtain a developer key.

- Go to WeatherUnderground site (Figure 18) the following

URL http://www.wunderground.com/weather/api/

- Select, Sign Up for FREE 


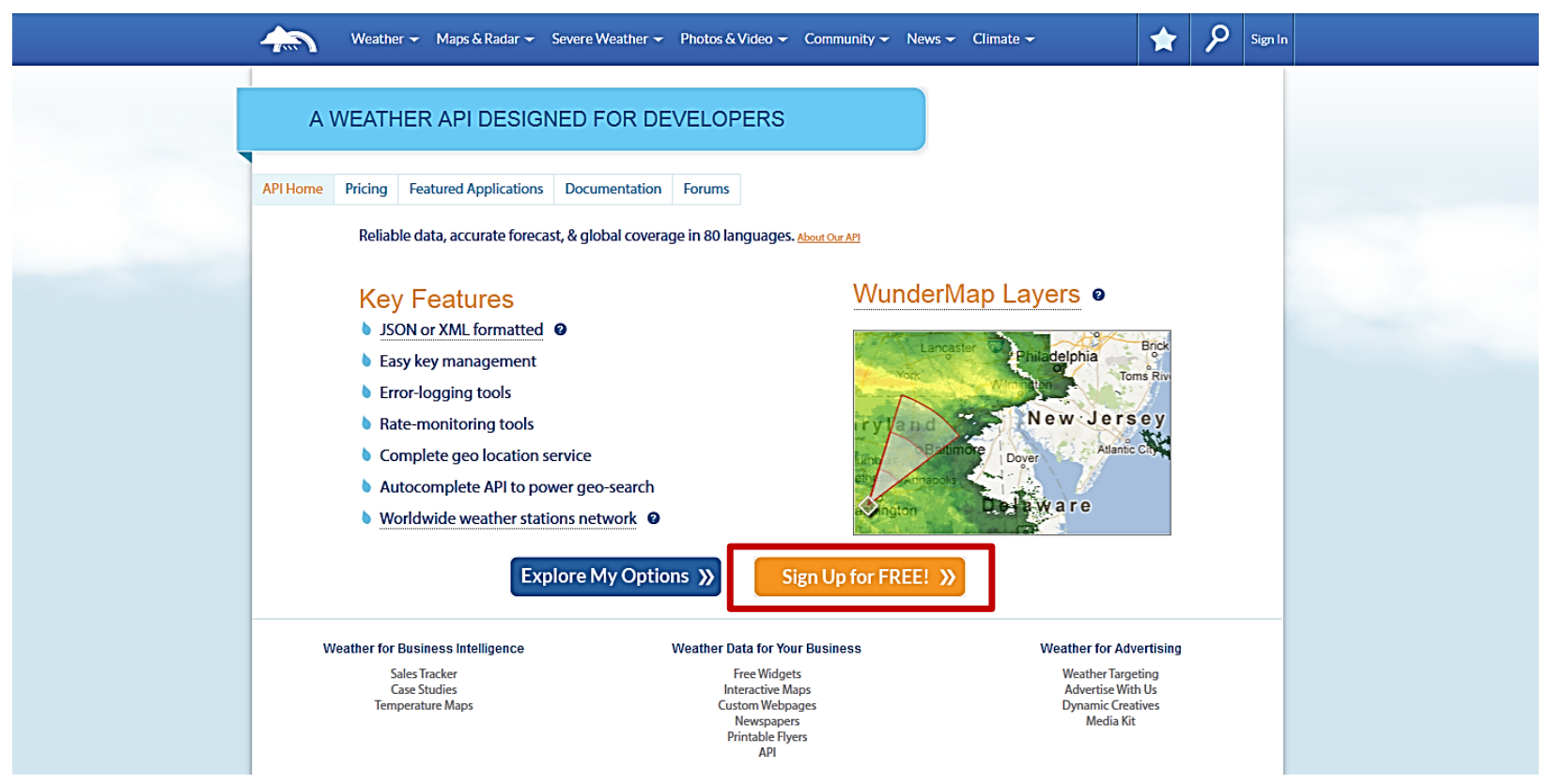

Figure 18: WeatherUnderground Website

- The window should now look similar to Figure 19. Enter your information to create an account.

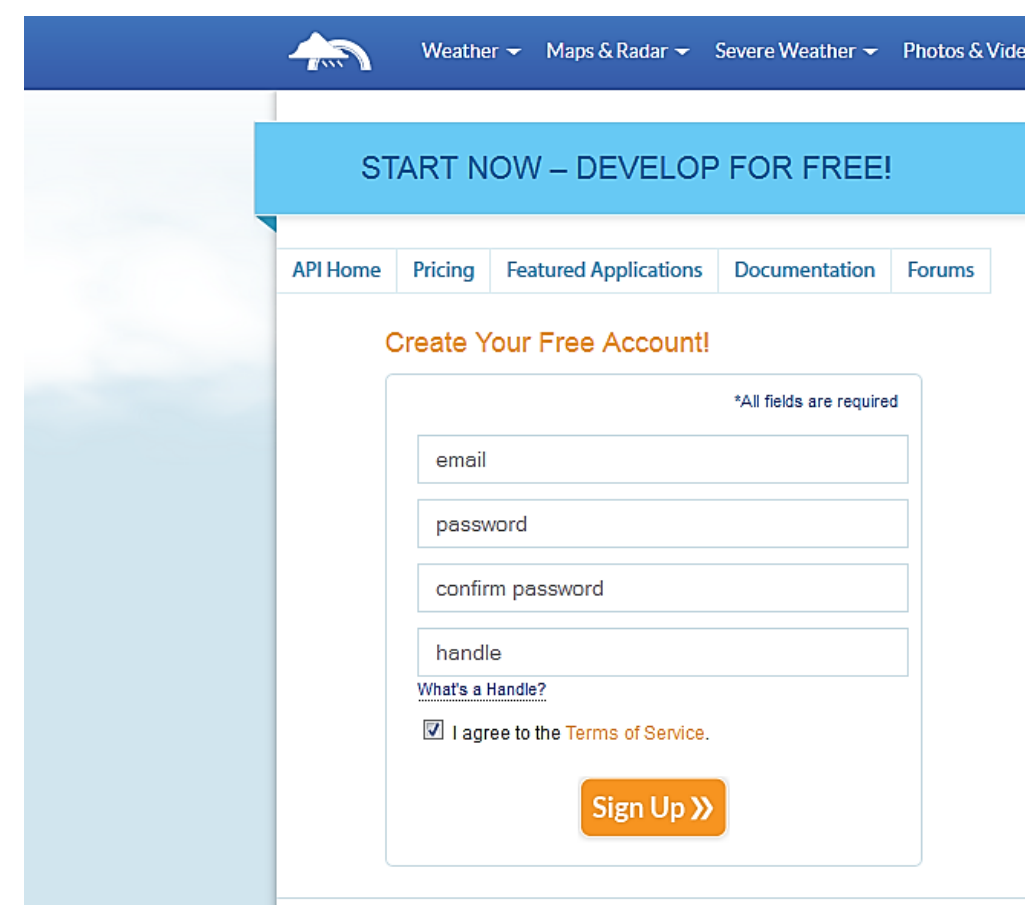

Figure 19: Setting up a Developer Account

- Select a plan that meets your needs. Login to with your username and password and click on "Explore my options button." For most applications the free plan will be adequate. The window should appear similar to Figure 20: 


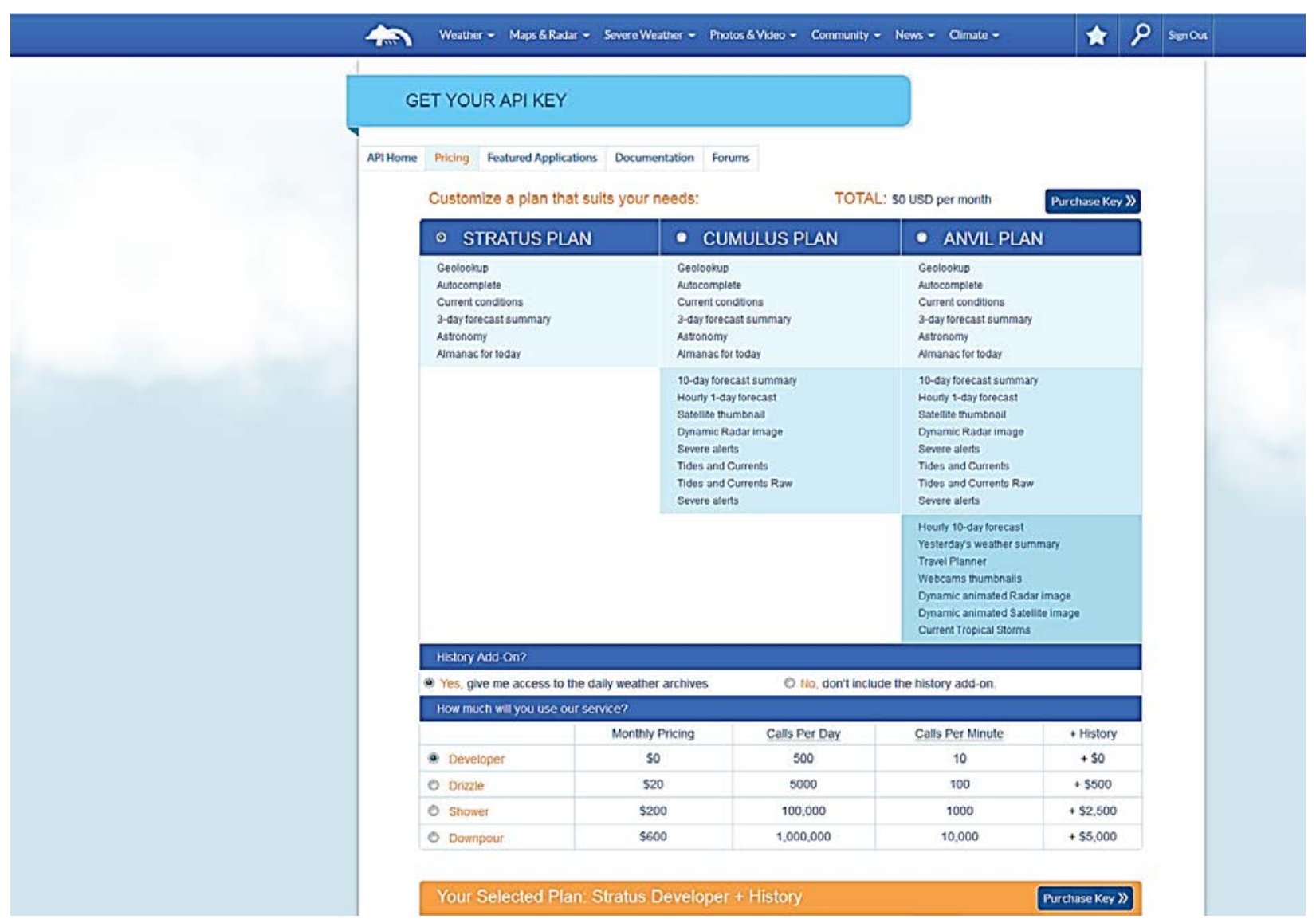

Figure 20: Creating a WeatherUnderground API Key

- You now have access to you WeatherUnderground API key. An example API key is shown in the red box of Figure 21:

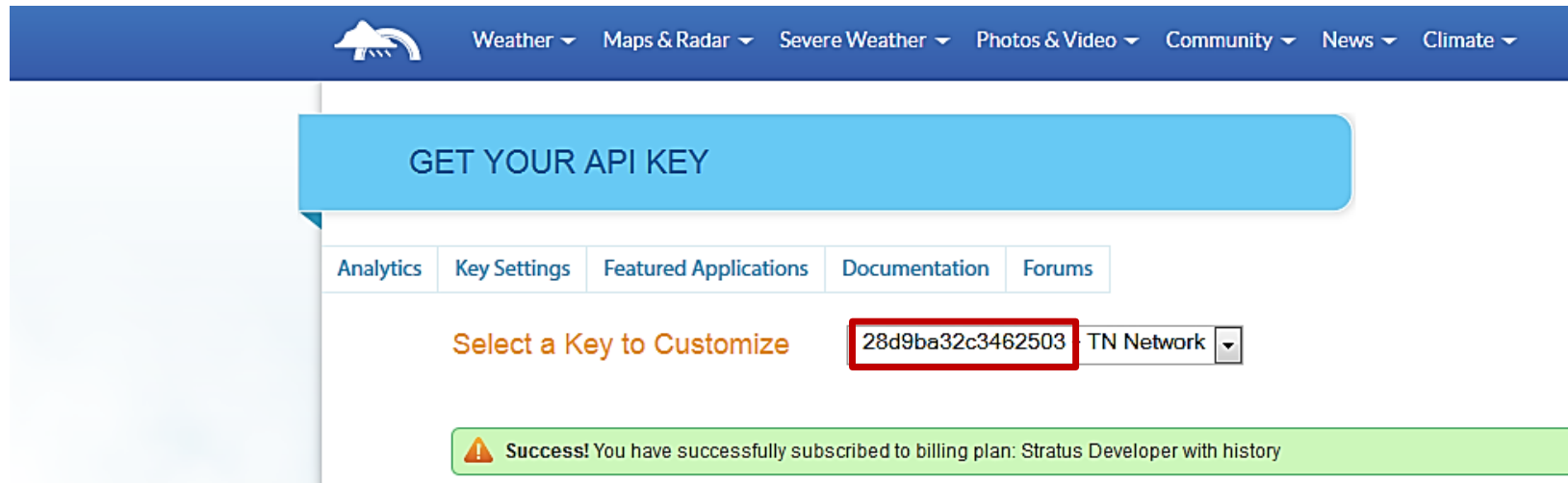

Figure 21: WeatherUnderground API Key

\subsubsection{Configuring WeatherAgent with Developer Key and Location}

The following steps will show how to configure the Weather agent with the developer key from WeatherUnderground and how to enter a zip code to get weather data from that zip code.

Edit Agents/WeatherAgent/weather/settings.py with your WeatherUnderground key. From the volttron directory enter the following terminal commands: 
1. Go to WeatherAgent directory:

- cd Agents

- cd WeatherAgent

- cd weather

2. Open settings.py with a text editor or nano:

\section{- nano settings.py}

3. Enter the key, as shown in Figure 22:

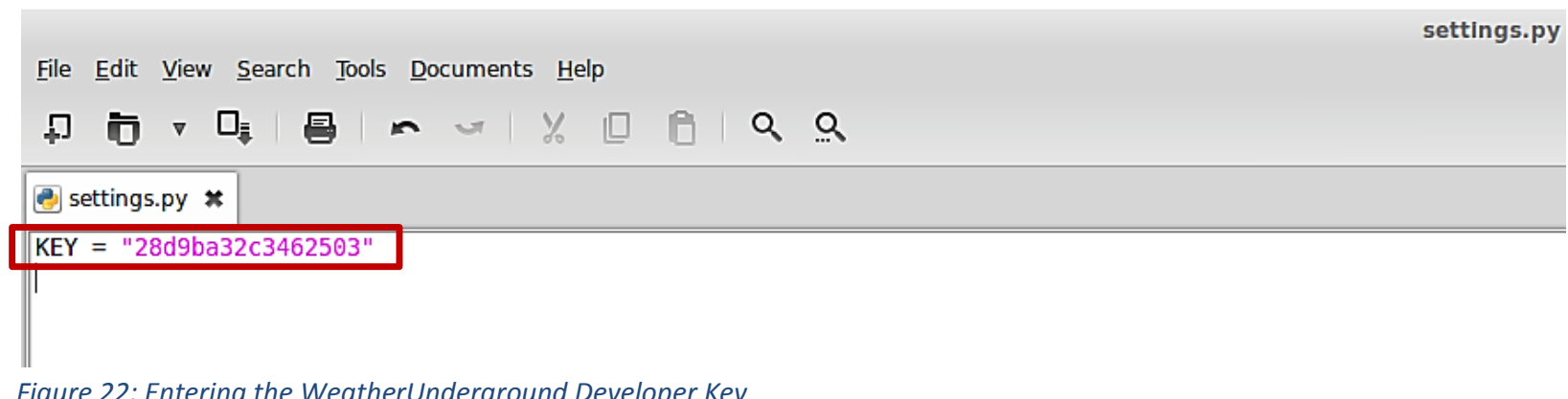

Figure 22: Entering the WeatherUnderground Developer Key

4. Open the weather agent's configuration file, Agents/WeatherAgent/weather-deploy.service, and edit “zip” field, as shown in Figure 23:

- nano weather-deploy.service

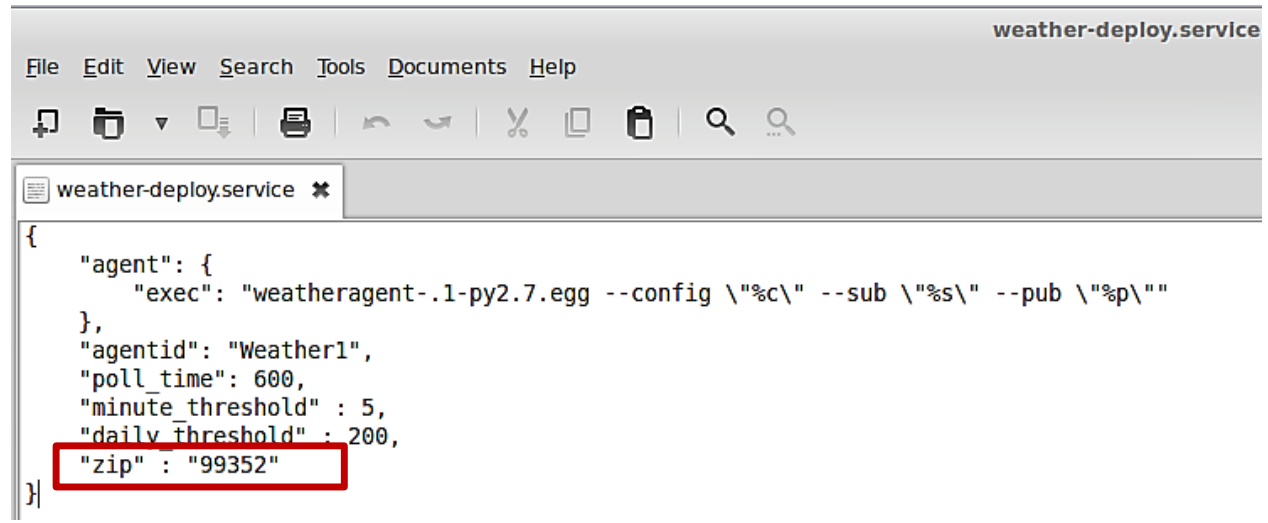

Figure 23: Entering Zip Code for the Location

\subsubsection{Launching the Weather Agent}

To launch the Weather agent, enter the following commands from the volttron directory:

1. Build the Weather agent eggsecutable:

\section{- volttron/scripts/build-agent.sh WeatherAgent}

2. Change permissions on the eggsecutable file: 
- $\quad$ chmod +x Agents/weatheragent-.1-py2.7.egg

3. Install the eggsecutable:

- $\quad$ bin/volttron-ctrl install-executable Agents/weatheragent-.1-py2.7.egg

4. Load the agent's configuration file:

\section{- bin/volttron-ctrl load-agent Agents/WeatherAgent/weather-deploy.service}

5. Start the agent:

\section{bin/volttron-ctrl start-agent weather-deploy.service}

6. Show the Weather agent's output, from the volttron.log:

\section{- tail volttron.log}

If changes are made to the Weather agent's settings file or configuration file after the agent is launched it is necessary to stop and reload the agent for those changes to take effect. See section 4.5 .5 on instructions for reloading an agent.

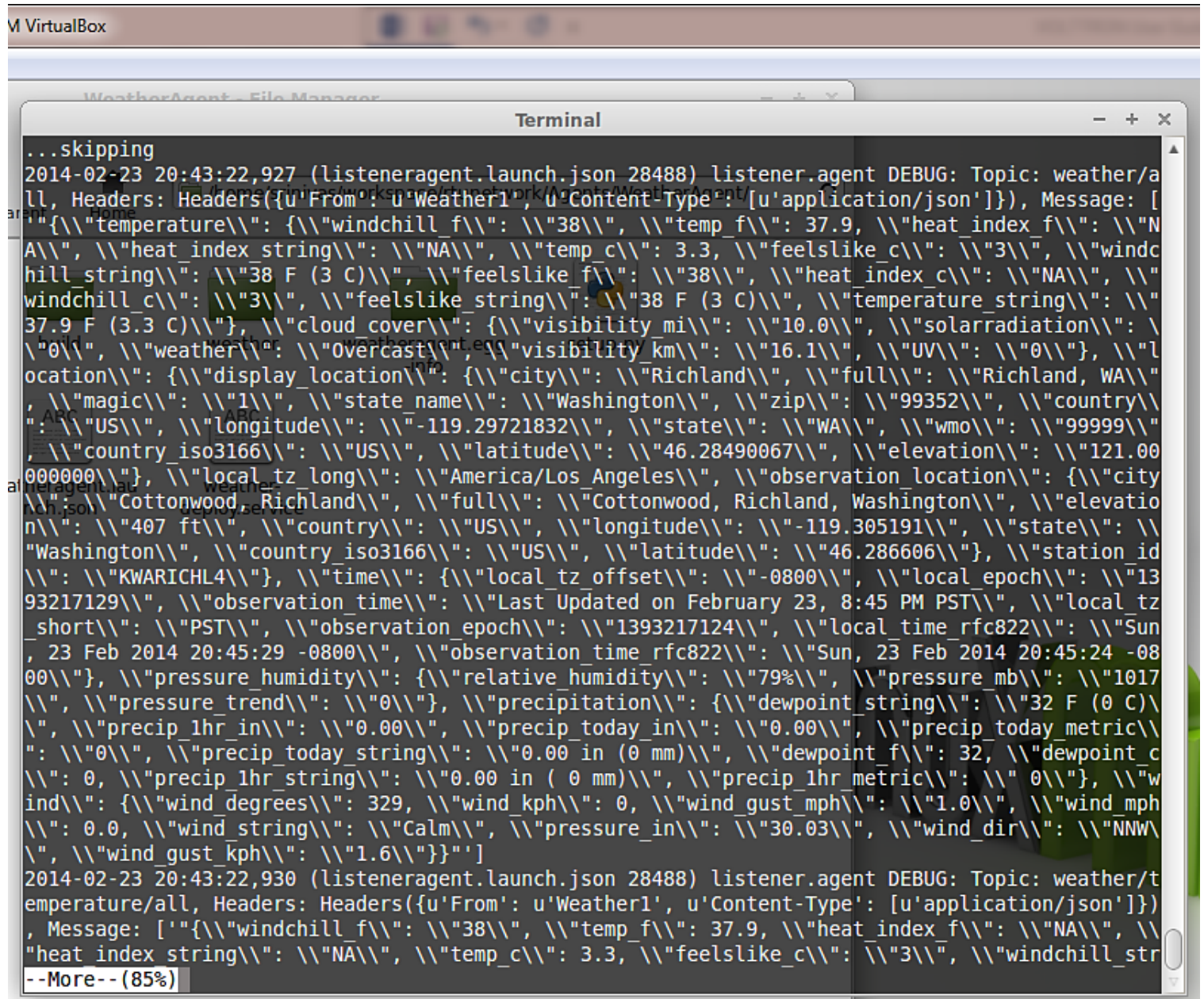

Figure 24: Example Output from the Weather Agent 


\subsection{Configuring and Launching sMAP Driver}

The sMAP driver allows a user to store time series data in the sMAP historian and to communicate with Modbus and BACnet compliant devices. Configuring the driver consists of creating a Modbus and/or a BACnet registry files and creating a sMAP driver configuration file. The Modbus and BACnet registry files tell the driver what the Modbus address is for each register, what data type it can hold, and if the register is writeable or read-only. The sMAP driver configuration file tells the sMAP driver where to find the Modbus and BACnet registry files, the location (URL) of the sMAP historian to write data to, and information related to the device you are monitoring or controlling.

\subsubsection{Configuring sMAP driver}

The basic Modbus commands can instruct a device to change a value in one of its registers, control or read an I/O port, as well as command the device to send back one or more values contained in its registers. To utilize Modbus communications for a device, a key of the registers must be constructed. This key is a file, in comma separated value format, that contains the point name that is published on the message bus, the I/O type (Modbus or BACnet register), and the point/register address on the device (point address). An example of a Modbus registry file is shown in Figure 25, and an example BACnet registry file is shown in Figure 26.

1. Create the Modbus registry file and the BACnet registry file.

\begin{tabular}{|c|c|c|c|c|c|c|}
\hline PNNL Point Name & Units & Units Details & Modbus Register & Writable & Point Address & Notes \\
\hline ReturnAirCO2 & PPM & $0.00-2000.00$ & >f & FALSE & 1001 & CO2 Reading $0.00-2000.0 \mathrm{ppm}$ \\
\hline SupplyfanSpeed & $\%$ & 0.00 to 100.00 & $>f$ & FALSE & 1003 & Fan speed from drive \\
\hline CoolsupplyFanSpeed1 & $\%$ & 0.00 to 100.00 ( 75 default) & $>t$ & TRUE & 1005 & Fan speed on cool 1 call \\
\hline CoolsupplyfanSpeed 2 & $\%$ & 0.00 to 100.00 (90 default) & $>t$ & TRUE & 1007 & Fan speed on Cool2 Call \\
\hline DischargeAirTemperature & $\mathrm{F}$ & (-) 39.99 to 248.00 & $>f$ & FALSE & 1009 & Discharge air reading \\
\hline ReturnAirCO2Stpt & PPM & 1000.00 (default) & $>f$ & TRUE & 1011 & Setpoint to enable demand control ventilation \\
\hline Dampersignal & $\%$ & $0.00-100.00$ & sf & FALSE & 1023 & Output to the economizer damper \\
\hline MixedAirTemperature & $F$ & (-) 39.99 to 248.00 & if & FALSE & 1025 & Mixed Air Temperature from Probe \\
\hline OutsideAirTemperature & $\mathrm{F}$ & $(-) 39.99$ to 248.00 & $>f$ & FALSE & 1029 & Outside Air Temperature \\
\hline OudoorAirVolume & $\%$ & 0.00 to 100.00 & $>f$ & FALSE & 1031 & Outside air volume claculated by multiplying damper and fan speed \\
\hline ReturnAirTemperature & $\mathrm{F}$ & $(-) 39.99$ to 248.00 & >t & FALSE & 1037 & Return air temperatuer reading \\
\hline Dampercommand & $\%$ & 0.100 & $>f$ & TRUE & 1059 & Damper position from Voltron to CATALYST \\
\hline MinimumDamperPositionstPt & $\%$ & 0.100 & $>f$ & TRUE & 1063 & Damper Minimum Postion Set to CATALYST from Voltron \\
\hline HeatingTemperaturestPt & $\mathrm{F}$ & $0-100$ & >f & TRUE & 1065 & Heating setpoint sent to the CATALYST from Voltron \\
\hline CoolingTemperaturestpt & f & $0-100$ & $>f$ & TRUE & 1067 & Cooling setpoint sent to the CATALYST from Voltron \\
\hline
\end{tabular}

Figure 25: An Example Modbus Registry File

\begin{tabular}{|c|c|c|c|c|c|c|c|}
\hline PNNL Point Name & Units & Unit Details & BACnet Object Type & Property & Writable & Index & Notes \\
\hline DischargeAirStaticPressure & inchesofWater & -0.20 to 5.00 & analoginput & presentvalue & FALSE & 3000108 & Resolution: 0.001 \\
\hline DischargeAirTemperature & degreesfahrenheit & -50.00 to 250.00 & analoginput & presentvalue & FALSE & 3000109 & Resolution: 0.1 \\
\hline MixedAirTemperature & degreesfahrenheit & -50.00 to 250.00 & analoginput & presentvalue & FALSE & 3000116 & Resolution: 0.1 \\
\hline OutdoorAirHumidity & percentRelativeHumidity & 0.00 to 100.00 & analoginput & presentvalue & FALSE & 3000117 & Resolution: 0.1 \\
\hline PreheatTemperature & degreesfahrenheit & -50.00 to 250.00 & analoginput & presentvalue & FALSE & 3000119 & Resolution: 0.1 \\
\hline ReturnAirTemperature & degreesfahrenheit & -50.00 to 250.00 & analoginput & presentvalue & FALSE & 3000120 & Resolution: 0.1 \\
\hline ReturnAirHumidity & percentRelativeHumidity & 0.00 to 100.00 & analoginput & presentvalue & FALSE & 3000124 & Resolution: 0.1 \\
\hline CoolingValveOutputCommand & percent & 0.00 to 100.00 (default 0.0 ) & analogOutput & presentvalue & TRUE & 3000107 & Resolution: 0.1 \\
\hline MixedAirDamperOutputCommand & percent & 0.00 to 100.00 (default 0.0 ) & analogOutput & presentvalue & TRUE & 3000110 & Resolution: 0.1 \\
\hline PreheatValveOutputCommand & percent & 0.00 to 100.00 (default 0.0 ) & analogOutput & presentvalue & TRUE & 3000111 & Resolution: 0.1 \\
\hline ReheatValveOutputCommand & percent & 0.00 to 100.00 (default 0.0 ) & analogOutput & presentvalue & TRUE & 3000112 & Resolution: 0.1 \\
\hline SupplyFanSpeedOutputCommand & percent & 0.00 to 100.00 (default 0.0 ) & analog Output & presentvalue & TRUE & 3000113 & Resolution: 0.1 \\
\hline ReturnFanSpeedOutputcommand & percent & 0.00 to 100.00 (default 0.0 ) & analogOutput & presentvalue & TRUE & 3000122 & Resolution: 0.1 \\
\hline
\end{tabular}

Figure 26: An Example BACnet Registry File

The data fields boxed in red in Figure 25 and Figure 26 are important for communication with the device(s) and/or control of the device(s). The fields boxed in blue are for informational purposes and are not required but often helpful, especially when using a registry key constructed by a third party. Save this file inside the workspace (i.e., /workspace/volttron/volttron/drivers/modbus.csv). 
For more details on the Modbus registry file or BACnet registry file visit the Transactional Network Wiki:

Modbus - https://github.com/VOLTTRON/volttron/wiki/ModbusDriver

BACnet - https://github.com/VOLTTRON/volttron/wiki/BacnetDriver

For information on auto-generating a BACnet registry file visit the Transactional Network Wiki:

https://github.com/VOLTTRON/volttron/wiki/AutoBacnetConfigGeneration

2. Configure the sMAP configuration file, as shown in Figure 27. 
[report 0]

\#Insert your SMAP key after add

ReportDeliveryLocation = http://smap.lbl.gov/backend/add/〈INSERT YOUR KEY HERE>

[/datalogger]

type $=$ volttron.drivers.data_logger.DataLogger

interval $=1$

[/]

type $=$ Collection

Metadata/SourceName $=$ 〈PUT YOUR NAME HERE>

uuid $=\langle$ PUT YOUR UUID HERE $>$

[/campus1]

type $=$ Collection

Metadata/Location/Campus = Campus Number 1

[/campus1/building1]

type $=$ collection

Metadata/Location/Building = Building Number 1

[/campus1/building1/modbus_device1]

type $=$ volttron.drivers.modbus. Modbus

ip_address = <PUT YOUR MODBUS DEVICE IP HERE $>$

Metadata/Instrument/Manufacturer = 〈PUT INSTRUMENT MANUFACTURER HERE $>$

Metadata/Instrument/ModelName = 〈PUT INSTRUMENT MODEL HERE >

slave id = <device slave id>

\#see volttron/drivers/example.csv for an example of a modbus register config file

register_config = <PUT YOUR REGISTER CONFIG HERE>

[/campus1/building1/bacnet_device1]

type = volttron.drivers.bacnet. BACnet

target_ip_address $=\langle$ PUT YOUR BACNET DEVICE IP HERE $>$

target_port $=$ <PUT YOUR BACNET DEVICE PORT HERE: DEFAULT 47808>

self_ip_address = 〈PUT IP OF INTERFACE USED TO COMMUNICATE WITH DEVICE (THIS COMPUTER IP) $>$

self_port $=$ <PUT PORT TO USE TO COMMUNICATE WITH DEVICE: DEFAULT 47808>

Metadata/Instrument/Manufacturer = 〈PUT INSTRUMENT MANUFACTURER HERE>

Metadata/Instrument/ModelName = 〈PUT INSTRUMENT MODEL HERE >

\#see volttron/drivers/bacnet_example_config.csv for example of BACnet registry file

register_config = <PUT YOUR REGISTER CONFIG HERE>

[/campus1/building1/logger]

\#write to the file specified.

type $=$ volttron.drivers.smap_logging.Logger

file = 'test.log'

Figure 27: An Example sMAP Configuration File

The required information is shown below:

The sMAP URL (location) and sMAP key 
-

sMAP metadata information

- /campus/building - path for publishing and subscribing to device data on the VOLTTRON message bus

- Modbus configurable parameters:

o Device IP address and slave identification (if applicable)

o Location of Modbus registry file

o Desired device metadata

- BACnet configurable parameters:

o Device IP address

o Location of BACnet registry file

o Desired device metadata

- The path to the data logger scripts. This information shows the sMAP driver where to find the logging code. The logging code allows an agent to publish information to the logging topic, where that information will then be pushed to sMAP. Copy this text and add it to your sMAP driver file.

3. Save the file within the transactional network workspace. Subsequent examples from this document will assume the file is saved as ( workspace/volttron/sMAP.ini).

\subsubsection{Launching the driver}

After configuring the Modbus registry key and the sMAP cofiguration file, the driver can now be launched. From a terminal window in the volttron directory, enter the following commands:

4. Activate the project:

- $\quad$ bin/activate (note the space after the period)

5. Launch the sMAP driver:

\section{- $\quad$ twistd -n smap sMAP.ini}

Keep this terminal window open. The driver will continue to run and allow you to interact with the device through the Actuator agent.

\subsection{Configuring and Launching the Actuator Agent}

The value contained in the registers on your Modbus or BACnet device will be published to the message bus at a regular interval (the read interval set in the sMAP configuration file). For on demand data or active control of the device, the Actuator agent must be configured and launched. The Actuator agent performs the following platform services: 
- Device control: The Actuator agent will accept commands from other agents and issue the commands to the specified device. Currently, communication with Modbus and BACnet compatible devices is supported.

- Device access scheduling: This service allows the scheduling of agents' access to devices to prevent multiple agents from controlling the same device at the same time.

\subsubsection{Configuring the Actuator Agent}

Before launching the Actuator agent, we must create or modify the Actuator agent's configuration file (Figure 28).

\{

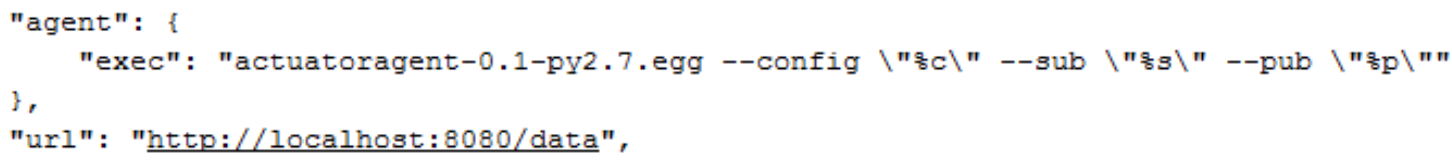

Figure 28: Example Actuator Agent Configuration File

The configuration parameters shown in Figure 28 are defined as follows:

- schedule_publish_interval - The interval in seconds between schedule announcements for devices being managed by the Actuator agent. These are the devices configured within the sMAP driver and the published schedule shows agent access information including which agent has scheduled access to the device.

- preempt_grace_time - The amount of time given to an application of "low" and "preemptable" priority when a higher priority application requests access to the device.

- schedule_state_file - Saved schedule information for each device being managed by the Actuator agent. 
- The parameters shown in the black box (in Figure 28) are related to construction of the agent eggsecutable. These parameters do not require modification.

- The parameters shown in the red box (in Error! Reference source not found.) are related to a device heartbeat. This is a register on the control device that the Actuator agent toggles to indicate proper communication with the platform has been established. The fields in red should be left blank unless your device has this register set up.

The Actuator agent will be able to facilitate communication and control of devices that are configured within the sMAP driver.

\subsubsection{Scheduling a Task}

To have active control of a device, an agent can request a block of time be scheduled on the device. An agent can request a task be scheduled by publishing to the "RTU/actuators/schedule/request" with the following header:

\{

'type': 'NEW_SCHEDULE',

'requesterID': <Agent ID>, \#The name of the requesting agent.

'taskID': <unique task ID>, \#unique ID for scheduled task.

'priority': <task priority>, \#The desired task priority, must be 'HIGH', 'LOW', or 'LOW_PREEMPT'

\}

The schedule request message should be formatted as follows (before converting to json):

[

["campus/building/device1", \#First time slot.

"2013-12-06 16:00:0९", \#Start of time slot.

"2013-12-06 16:20:๑९"], \#End of time slot.

["campus/building/device1", \#Second time slot.

"2013-12-06 18:00:00", \#Start of time slot.

"2013-12-๑6 18:20:๑९"], \#End of time slot.

["campus/building/device2", \#Third time slot.

"2013-12-06 16:00:०९", \#Start of time slot.

"2013-12-06 16:20:๑७"], \#End of time slot.

\#etc...

]

When constructing a schedule request for a device, the following should be noted:

- $\quad$ Everything in the header is required.

- A task schedule must have at least one time slot. 
- $\quad$ The start and end times are parsed with dateutil's date/time parser. The default string representation of a python datetime object will parse without issue.

- Two tasks are considered conflicted if at least one time slot on a device from one task overlaps the time slot of the other on the same device.

- The start or end (or both) of a requested time slot on a device may touch other time slots without overlapping and will not be considered in conflict.

- A request must not conflict with itself.

A schedule block of time and the associated task can have three possible priorities, as noted below:

HIGH - This task cannot be preempted under any circumstance. This task may preempt other conflicting preemptable tasks.

LOW - This task cannot be preempted once it has started. A task is considered started once the earliest time slot on any device has been reached. This task may not preempt other tasks.

LOW_PREEMPT - This task may be preempted at any time. If the task is preempted once it has begun running, any current time slots will be given a grace period (configurable in the Actuator agent configuration file, defaults to 60 seconds) before being revoked. This task may not preempt other tasks.

\subsubsection{Canceling a Task}

A task may be canceled by publishing to the "RTU/actuators/schedule/request" topic with the following header:

\{

'type': 'CANCEL_SCHEDULE',

'requesterID': <Agent ID>, \#The name of the requesting agent.

'taskID': <unique task ID>, \#ID of task being canceled

\}

When canceling a task, the following should be noted:

- The requesterID and taskID must match the original values from the original request header.

- After a task's time has passed, there is no need to cancel it. Doing so will result in a "TASK_ID_DOES_NOT_EXIST" error.

\subsubsection{Actuator Error Reply}

If something goes wrong, the Actuator agent will reply to both get and set on the error topic for an actuator:

'RTU/actuators/error/<full device path>/<actuation point $>$ '

With this header: 


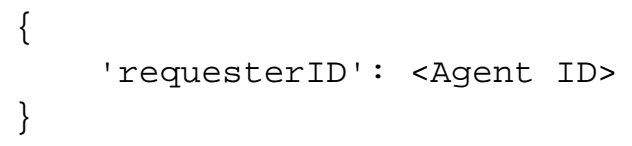

The message will be in the following form:

\{

'type': <Error Type or name of the exception raised by the request>

'value': <specific info about the error> \}

2.10.5 Task Preemption and Schedule Failure

In response to a task schedule request, the Actuator agent will respond on the topic

"RTU/actuators/schedule/response" with the following header:

\{

'type' : <'NEW_SCHEDULE', 'CANCEL_SCHEDULE'>

'requesterID': <Agent ID from the request>,

'taskID': <Task ID from the request>

\}

And, the following message (after parsing the json):

\{

'result': <'SUCCESS', 'FAILURE', 'PREEMPTED'>,

'info': <Failure reason, if any>,

'data': <Data about the failure or cancellation, if any> \}

2.10.5.1 Preemption Message

If a higher priority task preempts another scheduled task, the Actuator agent will publish the following message (the field type within the header will be contain CANCEL_SCHEDULE):

\{

'agentID': <Agent ID of preempting task>,

'taskID': <Task ID of preempting task>

\}

2.10.5.2 Failure Reasons

In most cases the Actuator agent will try to give good feedback as to why a request failed.

2.10.5.3 Failure Responses from Actuator Agent

The following list contains possible errors messages an agent may receive from the Actuator agent. This field corresponds to the info within the Actuator agent response message:

INVALID_REQUEST_TYPE - Request type was not "NEW_SCHEDULE" or "CANCEL_SCHEDULE".

MISSING_TASK_ID - Failed to supply a taskID. 
MISSING_AGENT_ID - AgentID not supplied.

TASK_ID_ALREADY_EXISTS - The supplied taskID already belongs to an existing task.

MISSING_PRIORITY - Failed to supply a priority for a task schedule request.

INVALID_PRIORITY - Priority not one of "HIGH", "LOW", or "LOW_PREEMPT".

MALFORMED_REQUEST_EMPTY - Request list is missing or empty.

REQUEST_CONFLICTS_WITH_SELF - Requested time slots on the same device overlap.

MALFORMED_REQUEST - Reported when the request parser raises an unhandled exception. The exception name and info are appended to this info string.

CONFLICTS_WITH_EXISTING_SCHEDULES - Schedule conflicts with an existing schedule that it cannot preempt. The data item for the results will contain info about the conflicts in this form (after parsing json):

\{

'<agentID1>' :

\{

'<taskID1>' :

[

["campus/building/device1",

"2013-12-๑6 16:0๑:๑९",

"2013-12-06 16:20:00"],

["campus/building/device1",

"2013-12-06 18:00:00",

"2013-12-06 18:20:00"]

]

$'<$ taskID2>' $:[\ldots]$

\}

'<agentID2>': $\{\ldots\}$

\}

TASK_ID_DOES_NOT_EXIST - Trying to cancel a task which does not exist. This error can also occur when trying to cancel a finished task.

AGENT_ID_TASK_ID_MISMATCH - A different agent ID is being used when trying to cancel a task

\subsubsection{Actuator Agent Interaction}

Once a task has been scheduled and the time slot for one or more of the devices has started, an agent may interact with the device using the get and set topics. Both get and set receive the same response from the Actuator agent.

\subsubsection{Getting values}

While the sMAP driver will periodically broadcast the state of a device, you may want an up-to-themoment value for an actuation point on a device. To request a value, publish a message to the following topic:

'RTU/actuators/get/<full device path>/<actuation point >' 
With this header:

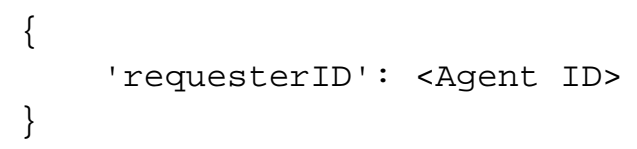

Values are set in a similar manner. To set a value, publish a message to the following topic:

'RTU/actuators/set/<full device path>/<actuation point>'

With this header:

\{

'requesterID': <Agent ID>

\}

The content of the message is the new, desired value for the actuation point.

2.10.6.3 Actuator Reply

The Actuator agent will reply to both get and set on the value topic for an actuator point:

'RTU/actuators/value/<full device path>/<actuation point $>$ '

With this header:

\{

'requesterID': <Agent ID>

\}

The message contains the value of the actuation point in JSON. The message can be parsed using jsonapi.loads method to parse to Python dictionary (from zmq.utils import jsonapi).

2.10.6.4 Common Error Types

The following list contains possible errors messages an agent may receive from the Actuator agent. This field corresponds to the info within the Actuator agent response message:

LockError - Returned when a request is made when we do not have permission to use a device. (Forgot to schedule, preempted and we did not handle the preemption message correctly, ran out of time in time slot, etc...)

ValueError - Message missing or could not be parsed as JSON.

Other error types involve problem with communication between the Actuator agent and sMAP.

2.10.7 Device Schedule State Announcements

Periodically the Actuator agent will publish the state of all currently used devices. For each device, the Actuator agent will publish to an associated topic:

'RTU/actuators/schedule/announce/<full device path>' 
With the following header:

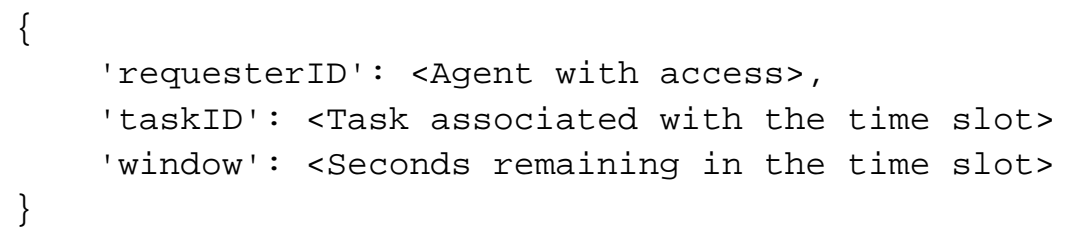

The frequency of the updates is configurable with the "schedule_publish_interval" setting.

2.10.8 Launching the Actuator Agent

After the Actuator agent has been configured, the agent can be launched. To launch the Actuator agent from the volttron directory, enter the following commands in a terminal window:

1. Build the Actuator agent eggsecutable:

\section{- volttron/scripts/build-agent.sh ActuatorAgent}

2. Change permissions on the eggsecutable file:

- $\quad$ chmod $+x$ Agents/actuatoragent-0.1-py2.7.egg

3. Install the eggsecutable:

- $\quad$ bin/volttron-ctrl install-executable Agents/ actuatoragent-0.1-py2.7.egg

4. Load the agent's configuration file:

\section{- bin/volttron-ctrl load-agent Agents/ActuatorAgent/actuator-deploy.service}

5. Start the agent:

\section{- bin/volttron-ctrl start-agent actuator-deploy.service}

6. Show the Actuator agent's output, from the volttron.log:

- cat volttron.log

If changes are made to the Actuator agent's configuration file after the agent is launched it is necessary to stop and reload the agent. See section 4.5 .5 on instructions for reloading an agent.

The Actuator agent can now be used to interact with Modbus devices or simulated devices. Any device, existing or not, can be scheduled. This can be a beneficial debugging tool especially when testing the functionality of an agent under development.

\subsubsection{Tips for Working with the Actuator Agent}

The following is a list of tips for working with the Actuator agent:

- An agent can watch the window value from device state announcements to perform scheduled actions within a time slot. 
o If an Agent's task is LOW_PREEMPT priority, it can watch for device state announcements, where the window is less than or equal to the grace period (default 60 seconds).

- When considering whether to schedule long or multiple short time slots on a single device:

o Do we need to ensure the device state for the duration between slots?

- Yes. Schedule one long time slot instead.

- No. Is it all part of the same task or can we break it up in case there is a conflict with one of our time slots?

- When considering time slots on multiple devices for a single task:

o Is the task really dependent on all devices or is it actually multiple tasks?

- When considering priority:

o Does the task have to happen on an exact day?

- No. Consider LOW and reschedule if preempted.

- Yes. Use HIGH.

o Is it problematic to prematurely stop a task once started?

- No. Consider LOW_PREEMPT and watch the device state announcements for a small window value.

- Yes. Consider LOW or HIGH.

- If an agent is only observing but needs to assure that no other task is going on while taking readings, it can schedule the time to prevent other agents from "messing" with a devices state. The device state announcements can be used as a reminder as to when to start watching. 


\section{Sample Applications/Agents}

This section summarizes the use of the sample applications that are pre-packaged with VOLTTRON. For detailed information on these applications, refer to the report Transactional Network Platform: Applications. ${ }^{7}$

\subsection{Automated Fault Detection and Diagnostic Agent}

The automated fault detection and diagnostic (AFDD) agent is used to identify problems in the operation and performance of air-handling units (AHUs) or packaged rooftop units (RTUs). Air-side economizers modulate controllable dampers to use outside air to cool instead of (or to supplement) mechanical cooling, when outdoor-air conditions are more favorable than the return-air conditions. Unfortunately, economizers often do not work properly, leading to increased energy use rather than saving energy. Common problems include incorrect control strategies, diverse types of damper linkage and actuator failures, and out-of-calibration sensors. These problems can be detected using sensor data used that is normally used to control the system.

The AFDD requires the following data fields to perform the fault detection and diagnostics: outside-air temperature, return-air temperature, mixed-air temperature, outside-air damper position/signal, supply fan status, mechanical cooling status, heating status. The AFDD supports both real-time data via a Modbus or BACnet device, or input of data from a csv style text document. If the data input is a csv text file, a "timestamp" containing date and time in the following format is required mm - dd - yyyy hh:mm

The following section will detail how to configure the AFDD agent, methods for data input (real-time data from a device or historical data in a comma separated value formatted text file), and launching the AFDD agent.

\subsubsection{Configuring the AFDD agent}

Before launching the AFDD agent several parameters require configuration. The AFDD utilizes the same JSON style configuration file that the Actuator, Listener, and Weather agents use, which is documented in the previous sections of this document. The threshold parameters used for the fault detection algorithms are pre-configured and will work well for most RTUs or AHUs. Figure 29 shows an example configuration file for the AFDD agent.

The parameters boxed in black (in Figure 29) are the pre-configured fault detection thresholds; these do not require any modification to run the AFDD agent. The parameters boxed in blue in the AFDD sample configuration file are related to creation of the AFDD agent eggsecutable and do not require modification. The parameters in the example configuration that are boxed in red will require user input. The following list describes each user configurable parameter and their possible values:

\footnotetext{
${ }^{7}$ http://www.pnl.gov/main/publications/external/technical_reports/PNNL-22941.pdf
} 
"agent": 1

"exec": "passiveafdd-0.1-py2.7.egg --config $\backslash " 8 c \backslash "--s u b \backslash " 8 s \backslash "--p u b \backslash " 8 p \backslash " n$

\},

"agentid": "afdd1",

"campus": "campus1",

"building": "building1",

"unit": "device1",

"gmap_path": "datalogger/log/afdd1/campus1/building1/device1" ,

\#/datalogger/log/your sMAP path here

\#[Controllex point names]

"oat_point_name": "OutsideAirTemp",

"mat_point_name": "MixedAirTemp", \#"DischargeAirTemp"

"dat_point_name": "DischargeAirTemperature",

"rat_point_name": "ReturnAirTemp",

"damper_point_name": "Damper",

"cool_cal11_point_name": "CoolCall",

"cool_cmd1_point_name": "CompressorStatus",

"fan_status_point_name": "FanStatus",

"heat_command1_point_name": "Heating",

\#[Input Variables]

"aggregate_data": 1 ,

"csv_input": 1 ,

"EER": 10,

"tonnage": 10

"high_1imit": 70,

"economizer_type": 0 ,

"matemp_missing": 0 ,

\section{\# [oaf]}

"oaf_temp_threshold": 4.0 ,

₹ [OAE1]

"mat_low": 50,

"mat_high": 90 ,

"rat_low": 50,

"rat_high": 90 ,

"oat_low": 30 ,

"oat_high": 120 ,

\# [OAE2]

"oae2_damper_threshold": 30.0,

"oae2_oaf_threshold": 0.25 ,

\#[OAE3]

"damper_minimum": 20 ,

\# [OAE4]

"minimum_oa": 0.1 ,

"oae4_oaf_threshold": 0.25 ,

\# [OAE5]

"oaes_oaf_threshold": 0.0 ,

\# [OAE6]

"Sunday": $[0,23]$, \#this schedule is 24 hours

"Monday": $[0,23]$,

"Tuesday" : $[0,23]$,

"Wednesday" : $[0,23]$,

"Thursday": $[0,23]$,

"Friday": $[0,23]$.

"Saturday": $[0,23]$,

Figure 29: Example AFDD Agent Configuration File 
agentid - This is the ID used when making schedule, set, or get requests to the Actuator agent; usually a string data type.

campus - Campus name as configured in the sMAP driver. This parameter builds the device path that allows the Actuator agent set and get values on the device; usually a string data type.

building - Building name as configured in the sMAP driver. This parameter builds the device path that allows the Actuator agent set and get values on the device; usually a string data type.

unit - Device name as configured in the sMAP driver. This parameter builds the device path that allows the Actuator agent set and get values on the device; usually a string data type.

Note: The campus, building, and unit parameters are used to build the device path

(campus/building/unit). The device path is used for communication on the message bus.

Controller point names - When using real-time communication, the Actuator agent identifies what registers or values to set or get by the point name you specify. This name must match the "Point Name" Given in the Modbus registry file, as specified in section 2.9.1 Configuring sMAP driver.

aggregate_data - When using real-time data sampled at an interval of less than 1 hour or when inputting data via a csv file sampled at less than 1 hour intervals, set this flag to "1." Should be an integer or floating point number (i.e., 1 or 1.0)

csv_input - Flag to indicate if inputting data from a csv text file. Set to " 0 ” for use with real-time data from a device or " 1 " if data is input from a csv text file. It should be an integer or floating point number (i.e., 1 or 1.0 )

EER - Energy efficiency ratio for the AHU or RTU. It should be an integer or floating point number (i.e., 10 or 10.0$)$

tonnage - Cooling capacity of the AHU or RTU in tons of cooling. It should be an integer or floating point number (i.e., 10 or 10.0)

economizer_type - This field indicates what type of economizer control is used. Set to " 0 ” for differential dry-bulb control or to "1" for high limit dry-bulb control. It should be an integer or floating point number.

high_limit - If the economizer is using high limit dry-bulb control, then this value will indicates what the outside-air temperature high limit should be. The input should be floating point number (i.e., 60.0)

matemp_missing - Flag used to indicate if the mixed-air temperature is missing for this system. If utilizing csv data input, simply set this flag to " 1 " and replace the mixed-air temperature column with discharge-air temperature data. If using real-time data input change the field “mat_point_name” under Point names section to the point name indicating the discharge-air temperature. It should be an integer or floating point number (i.e., 1 or 1.0) 
OAE6 - This section contains the schedule information for the AHU or RTU. The default is to indicate a 24-hour schedule for each day of the week. To modify this, change the numbers in the bracketed list next to the corresponding day with which you are making operation schedule modifications. For example:

"Saturday": [0,0] (This indicates the system is off on Saturdays)

\subsubsection{Launching the AFDD Agent}

The AFDD agent performs passive diagnostics on AHUs or RTUs, monitors and utilizes sensor data but does not actively control the devices. Therefore, the agent does not require interaction with the Actuator agent. Steps for launching the agent are as follows:

In a terminal window, enter the following commands:

1. Build the AFDD agent eggsecutable:

- volttron/scripts/build-agent.sh PassiveAFDD

2. Change permissions on the eggsecutable file:

- $\quad$ chmod +x Agents/passiveafdd-0.1-py2.7.egg

3. Install the eggsecutable:

- $\quad$ bin/volttron-ctrl install-executable Agents/passiveafdd-0.1-py2.7.egg

4. Load the agent's configuration file:

- bin/volttron-ctrl load-agent Agents/PassiveAFDD/passiveafdd.launch.json

5. Start the agent:

- bin/volttron-ctrl start-agent passiveafdd.launch.json

6. Show the AFDD agent's output, from the volttron.log:

- tail volttron.log

When the AFDD agent is monitoring a device via the message bus, the agent relies on the periodic data published from the sMAP driver. The AFDD agent then aggregates this data each hour and performs the diagnostics on the average hourly data. The result is written to a csv text file, which is appended if the file already exists. This file is in a folder titled "Results" under the "PassiveAFDD/passiveafdd/" directory. Below is a key that describes how to interpret the diagnostic results:

\begin{tabular}{|c|c|}
\hline Diagnostic & Code Message \\
\hline code & AFDD-1 (Temperature Sensor Fault) \\
\hline 20 & No faults detected \\
\hline 21 & Temperature sensor fault \\
\hline 22 & Conditions not favorable for diagnostic \\
\hline 23 & Mixed-air temperature outside of expected range \\
\hline
\end{tabular}


24 Return-air temperature outside of expected range

25 Outside-air temperature outside of expected range

27 Missing data necessary for fault detection

29 Unit is off (No Fault)

\section{AFDD-2 (RTU Economizing When it Should)}

$30 \quad$ No faults detected

31 Unit is not currently cooling or conditions are not favorable for economizing (No Fault) Insufficient outdoor air when economizing (Fault)

33 Outdoor-air damper is not fully open when the unit should be economizing (Fault)

36 Damper is open for economizing but conditions were not favorable for OAF calculaton (No Fault)

37 Missing data necessary for fault detection (No Fault)

38 Damper is fully open when economizing but OAF calculation led to an unexpected value (No Fault)

$39 \quad$ Unit is off (No Fault)

\section{AFDD-3(Unit Economizing When it Should)}

$40 \quad$ No faults detected

41 Damper should be at minimum position but is not (Fault)

42 Damper is at minimum for ventilation (No Fault)

43 Conditions favorable for economizing (No Fault)

$47 \quad$ Missing data necessary for fault detection (No Fault)

$49 \quad$ Unit is off (No Fault)

\section{AFDD-4(Excess Outdoor-air Intake)}

$50 \quad$ No faults detected

51 Excessive outdoor-air intake

52 Damper is at minimum but conditions are not favorable for OAF calculation (No Fault)

53 Damper is not at minimum (Fault)

$56 \quad$ Unit should be economizing (No Fault)

57 Missing data necessary for fault detection (No Fault)

58 Damper is at minimum but OAF calculation led to an unexpected value (No Fault)

$59 \quad$ Unit is off (No Fault)

\section{AFDD-5 (Insufficient Outdoor-air Ventilation)}

$60 \quad$ No faults detected

61 Insufficient outdoor-air intake (Fault)

62 Damper is at minimum but conditions are not favorable for OAF calculation (No Fault)

63 Damper is not at minimum when is should not be (Fault)

66 Unit should be economizing (No Fault)

67 Missing data necessary for fault detection (No Fault)

68 Damper is at minimum but conditions are not favorable for OAF calculation (No Fault)

69 Unit is off (No Fault) 


\section{AFDD-6 (Schedule)}

$70 \quad$ Unit is operating correctly based on input on/off time (No Fault)

$71 \quad$ Unit is operating at a time designated in schedule as "off" time

$77 \quad$ Missing data

\subsubsection{Launching the AFDD for CSV Data Input}

When utilizing the AFDD agent and inputting data via a CSV text file, set the csv_input parameter, contained in the AFDD configuration file, to " 1. "

- Launch the agent normally, as described in Section 3.1.2.

- A small file input box will appear. Navigate to the csv data file and select the csv file to input for the diagnostic.

- The result will be created for this RTU or AHU in the results folder described in Section Error! Reference source not found..

Figure 30 shows the dialog box that is used to input the CSV data file.

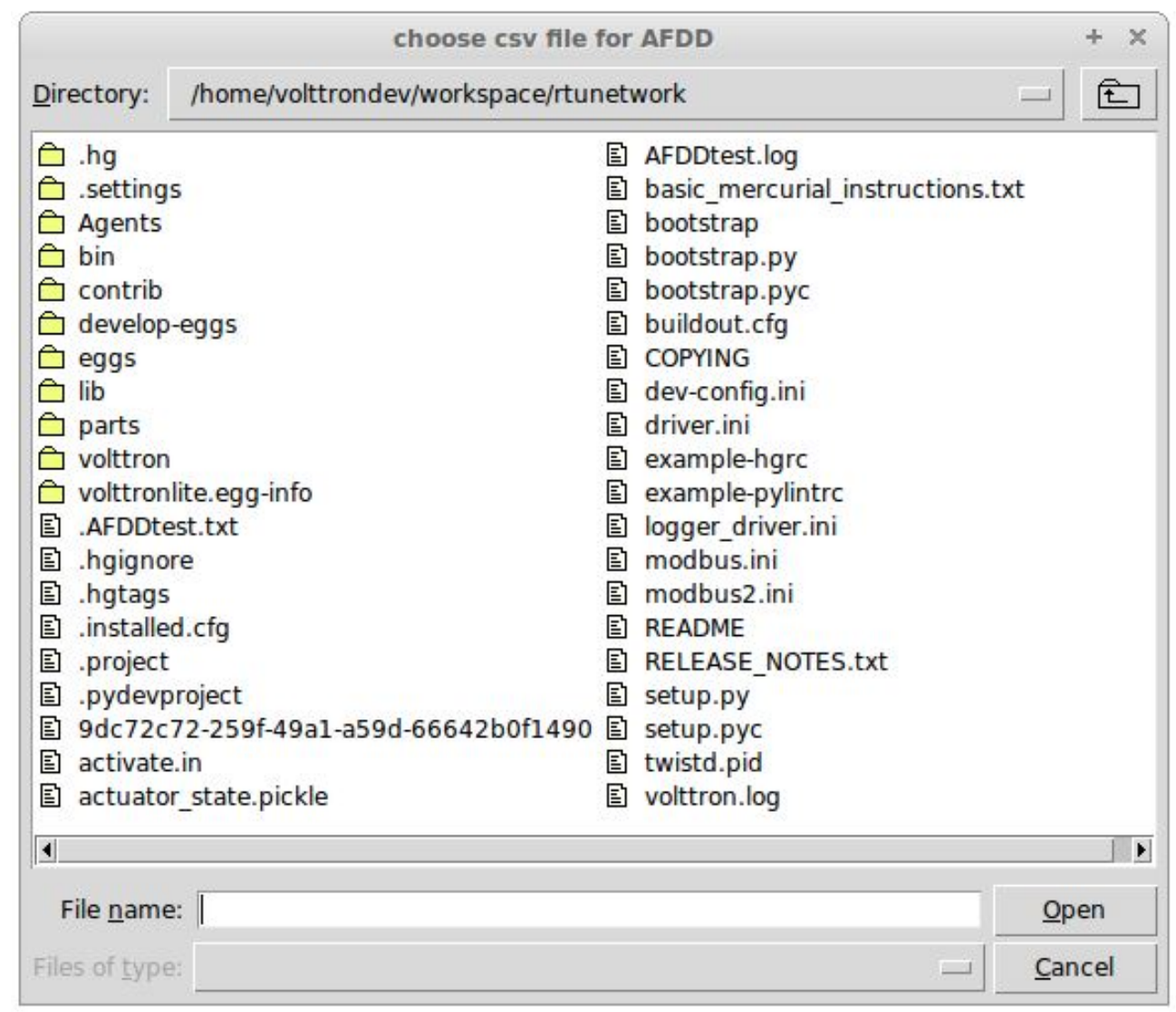

Figure 30: File Selection Dialog Box when Inputting Data in a CSV File

If "Cancel" is pushed on the file input dialog box, the AFDD will acknowledge that no file was selected. The AFDD must be restarted to run the diagnostics. If a non-CSV file is selected, the AFDD will acknowledge the file selected was not a CSV file. The AFDD must be restarted to run the diagnostics. 
To restart the AFDD, enter the commands in a terminal window from the volttron directory:

\section{- bin/volttron-ctrl stop-agent passiveafdd.launch.json}

\section{- bin/volttron-ctrl start-agent passiveafdd.launch.json.}

Note: This only restarts the agent. If modifications are also made to the passiveafdd.launch.json configuration file, then the AFDD configuration file must be unloaded and reloaded before the agent is restarted. For directions on reloading the agent, see Section 4.5.5.

Figure 31 shows a sample input data in a CSV format.

Timestamp, OutsideAirTemp, ReturnAirTemp, MixedAirTemp, CompressorStatus, HeatingStatus, FanStatus, Damper
5/19/2012 6:00,48.902,56.43727273,58.68472222,0,0,0,0
$5 / 19 / 20127: 00,51.12316667,59.47933333,59.58916667,0,0,0,0$
$5 / 19 / 20128: 00,54.70866667,61.1625,64.34266667,0,0,0,0$

Figure 31: Sample of CSV Data for AFDD Agent

Note that the column order is important. The data should be ordered as follows timestamp, outside-air temperature, return-air temperature, mixed-air temperature, mechanical cooling status, heating status, supply fan status, and outside-air damper signal. The header, or name for each column does not matter to the AFDD agent. Use a column name that is easy to identify and understand.

\subsection{The Demand Response (DR) Agent}

Many utilities around the country have or are considering implementing dynamic electrical pricing programs that use time-of-use (TOU) electrical rates. TOU electrical rates vary based on the demand for electricity. Critical peak pricing (CPP), also referred to as critical peak days or event days, is an electrical rate where utilities charge an increased price above normal pricing for peak hours on the CPP day. CPP times coincide with peak demand on the utility; these CPP events are generally called between 5 to 15 times per year and occur when the electrical demand is high and the supply is low. Customers on a flat standard rate who enroll in a peak time rebate program receive rebates for using less electricity when a utility calls for a peak time event. Most CPP events occur during the summer season on very hot days. The initial implementation of the DR agent addresses CPP events where the RTU would normally be cooling. This implementation can be extended to handle CPP events for heating during the winter season as well. This implementation of the DR agent is specific to the CPP, but it can easily be modified to work with other incentive signals (real-time pricing, day head, etc.).

The main goal of the building owner/operator is to minimize the electricity consumption during peak summer periods on a CPP day. To accomplish that goal, the DR agent performs three distinct functions:

Step 1 - Pre-Cooling: Prior to the CPP event period, the cooling and heating (to ensure the RTU is not driven into a heating mode) set points are reset lower to allow for pre-cooling. This step allows the RTU to cool the building below its normal cooling set point while the electrical rates are still low (compared to $\mathrm{CPP}$ events). The cooling set point is typically lowered between 3 and $5^{\circ} \mathrm{F}$ below the normal. Rather than 
change the set point to a value that is 3 to $5^{\circ} \mathrm{F}$ below the normal all at once, the set point gradually lowered over a period of time.

Step 2 - Event: During the CPP event, the cooling set point is raised to a value that is 4 to $50 F$ above the normal, the damper is commanded to a position that is slightly below the normal minimum (half the of the normal minimum), the fan speed is slightly reduced (by $10 \%$ to $20 \%$ of the normal speed, if the unit has a variable-frequency-drive (VFD)), and the second stage cooling differential (time delay between stage one and stage two cooling) is increased (by few degrees, if the unit has multiple stages). The modifications to the normal set points during the CPP event for the fan speed, minimum damper position, cooling set point, and second stage cooling differential are user adjustable. These steps will reduce the electrical consumption during the CPP event. The pre-cooling actions taken in step 1 will allow the temperature to slowly float up to the CPP cooling temperature set point and reduce occupant discomfort during the attempt to shed load.

Step 3 - Post-Event. The DR agent will begin to return the RTU to normal operations by changing the cooling and heating set points to their normal values. Again, rather than changing the set point in one step, the set point is changed gradually over a period of time to avoid the "rebound" effect (a spike in energy consumption after the CPP event when RTU operations are returning to normal).

The following section will detail how to configure and launch the DR agent.

\subsubsection{Configuring DR Agent}

Before launching the DR agent, several parameters require configuration. The DR utilizes the same JSON style configuration file that the Actuator, Listener, and Weather agent use. A notable limitation of the DR agent is the DR agent requires active control of an RTU/AHU. The DR agent modifies set points on the controller or thermostat to reduce electrical consumption during a CPP event. The DR agent must be able to set certain values on the RTU/AHU controller or thermostat via the Actuator agent (Section 2.10). Figure 32 shows a sample configuration file for the DR agent: 


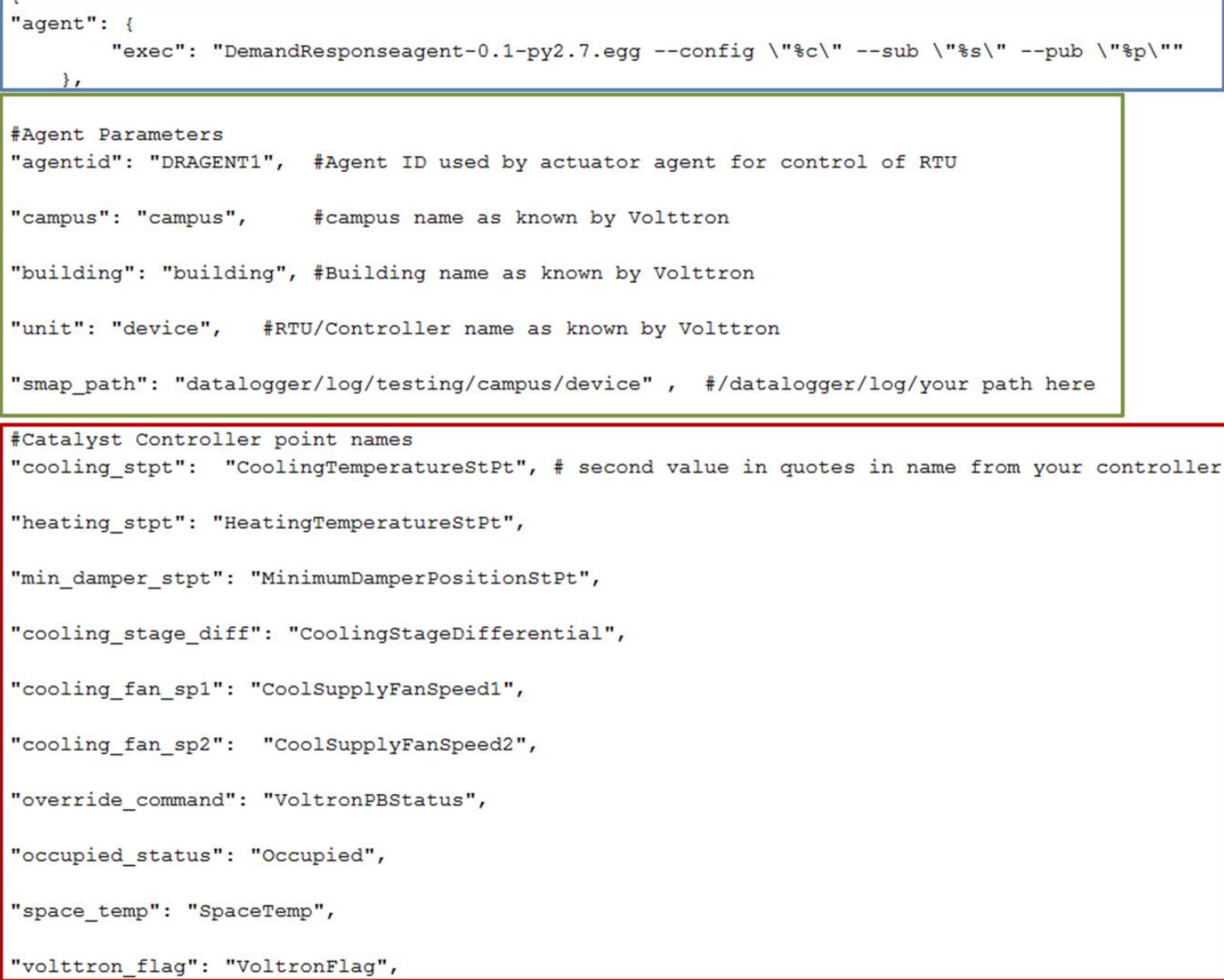

Figure 32: Example Configuration File for the DR Agent 
The parameters boxed in black (Figure 32) are the demand response parameters; these may require modification to ensure the DR agent and corresponding CPP event are executed as one desires. The parameters boxed in blue in the DR sample configuration file are related to the creation of the DR agent eggsecutable and do not require modification. The parameters in the example configuration that are boxed in red are the controller or thermostat points, as specified in the Modbus or BACnet (depending on what communication protocol your device uses) registry file, that the DR agent will set via the Actuator agent. These device points must be writeable, and configured as such, in the registry (Modbus or BACnet) file. The following list describes each user configurable parameter:

agentid - This is the ID used when making schedule, set, or get requests to the Actuator agent; usually a string data type.

campus - Campus name as configured in the sMAP driver. This parameter builds the device path that allows the Actuator agent set and get values on the device; usually a string data type.

building - Building name as configured in the sMAP driver. This parameter builds the device path that allows the Actuator agent set and get values on the device; usually a string data type.

unit - Device name as configured in the sMAP driver. This parameter builds the device path that allows the Actuator agent set and get values on the device; usually a string data type.

Note: The campus, building, and unit parameters are used to build the device path

(campus/building/unit). The device path is used for communication on the message bus.

csp_pre - Pre-cooling space cooling temperature set point.

csp_cpp - CPP event space cooling temperature set point.

normal_firststage_fanspeed - Normal operations, first stage fan speed set point.

normal_secondstage_fanspeed - Normal operations, second stage fan speed set point.

normal_damper_stpt - Normal operations, minimum outdoor-air damper set point.

normal_coolingstpt - Normal operations, space cooling temperature set point.

normal_heatingstpt - Normal operations, space heating temperature set point.

fan_reduction - Fractional reduction in fan speeds during CPP event (default: $0.1-10 \%$ ).

damper_cpp - CPP event, minimum outdoor-air damper set point.

max_precool_hours - Maximum allotted time for pre-cooling, in hours.

cooling_stage_differential - Difference in actual space temperature and set-point temperature before second stage cooling is activated. 
Schedule - Day of week occupancy schedule “ 0 ” indicate unoccupied day and " 1 ” indicate occupied day (e.g., $[1,1,1,1,1,1,1]=$ [Mon, Tue, Wed, Thu, Fri, Sat, Sun]).

\subsubsection{OpenADR (Open Automated Demand Response)}

Open Automated Demand Response (OpenADR) is an open and standardized way for electricity providers and system operators to communicate DR signals with each other and with their customers using a common language over any existing IP-based communications network, such as the Internet. Lawrence Berkeley National Laboratory created an agent to receive DR signals from an external source, e.g., OpenADR server, and publish this information on the message bus. The demand response agent subscribes to the OpenADR topic and utilizes the contents of this message to coordinate the CPP event.

The format of the OpenADR signal is formatted as follows:

'openadr/event',\{'Content-Type': ['application/json'], 'requesterID': 'openadragent'\}, '\{ "status": "near", "start_at": "2013-6-15 14:00:00", "end_at":"2013-10-15 18:00:00","mod_num":0,"id":"18455630-a5c44e4a-9d53-b3cf989ccf1b","signals":null\}'

The red text in the signal is the topic associated with CPP events that are published on the message bus. The text in dark blue is the message; this contains the relevant information on the CPP event for use by the DR agent.

If one desires to test the behavior of a device when responding to a DR event, one may simulate such an event by manually publishing a DR signal on the message bus. From the rutnetwork directory, in a terminal window, enter the following commands:

- Activate project:

0 . bin/activate (not the space after the period)

- $\quad$ Start Python interpreter:

o python (this activates the Python interpreter)

- Import PublishMixin module:

o from volttron.lite.agent.base import PublishMixin

- $\quad$ Create PublishMixin object:

o p=PublishMixin('ipc:///tmp/volttron-lite-agent-publish')

- Publish simulated OpenADR message:

o p.publish_json('openadr/event',\{\},\{'id': 'event_id','status': 'active', 'start_at': '0610-14 14:00', 'end_at': '06-10-14 18:00'\})

To cancel this event, enter the following command: 
- p.publish_json('openadr/event',\{\},\{'id': 'event_id','status': ‘cancelled', 'start_at': '06-10-14 14:00', 'end_at': '06-10-14 18:00'\})

The DR agent will use the most current signal for a given day. This allows utilities/OpenADR to modify the signal up to the time prescribed for pre-cooling.

\subsubsection{DR Agent Output to sMAP}

The Demand Response agent will output to the sMAP location prescribed in your sMAP configuration file. The specific "branch" within this sMAP database is specified in the DR agent's configuration file. The DR agent will output the start time for the CPP event and the end time for the CPP event. These will be specified by a value of " 1 " for the start time and " 2 " for the end time. If the CPP event is cancelled or a user override is initiated, the DR agent will push a value of "3" to sMAP.

\subsubsection{Launching the Demand Response Agent}

After the DR agent has been configured, the agent can be launched. To launch the DR agent from the volttron directory, enter the following commands in a terminal window:

- $\quad$ Build the DR agent eggsecutable:

o volttron/scripts/build-agent.sh DRAgent

- Change permissions on the eggsecutable file:

o chmod +x Agents/DemandResponseagent-0.1-py2.7.egg

- Install the eggsecutable:

o bin/volttron-ctrl install-executable Agents/DemandResponseagent-0.1-py2.7.egg

- Load the agent's configuration file:

0 bin/volttron-ctrl load-agent

Agents/DemandResponseAgent/demandrepsonseagent.launch.agent

- $\quad$ Start the agent:

o bin/volttron-ctrl start-agent listeneragent.launch.json

- Show the DR agent's output, from the volttron.log:

o cat volttron.log 


\section{Agent Development in VOLTTRON}

VOLTTRON supports agents written in any language. The only requirement is that they can communicate over the message bus. However, Python-based agents were the focus of the original work, and an array of utilities have been created to speed development in that language. The

“volttron.lite.agent” package contains these utilities ( /workspace/volttron/volttron/lite/agent.py). For the details of using them, please see the Wiki or read the comments in the code. These comments contain explanations and examples of usage.

The utility classes in this package, with a brief description, follow:

- base.py: BaseAgent class handles much of the low-level requirements for working in the platform. An agent that extends the BaseAgent can focus on its own functionality and override only the methods it needs.

- matching.py: Allows agents to easily subscribe to topics using decorators ${ }^{8}$

- cron.py: Allows agents to schedule their actions ahead of time

- green.py: Utilities for using greenlets ${ }^{9}$ with the BaseAgent

- sched.py: Used for scheduling objects

- utilities.py: Utilities for loading config files, parsing command line arguments, formatting log messages, etc.

The following sections will walkthrough an example agent and then give an overview of creating a simple agent from scratch.

\subsection{Example Agent Walkthrough}

The Listener agent subscribes to all topics and is useful for testing that agents being developed are publishing correctly. It also provides a template for building other agents because it utilizes publish and subscribe mechanisms and contains the basic structure necessary to build a very simple agent.

\subsection{Explanation of Listener Agent}

The Listener agent utilizes the PublishMixin and BaseAgent classes for its base functionality. Please see volttron/lite/agent/base.py for the details of these classes.

The Listener agent publishes a heartbeat message using the PublishMixin. It also extends BaseAgent to get default functionality such as responding to platform commands. When creating agents, Mixins ${ }^{10}$ should be first in the class definition.

\footnotetext{
${ }^{8}$ https://wiki.python.org/moin/PythonDecorators

${ }^{9}$ https://pypi.python.org/pypi/greenlet

${ }^{10}$ http://python.dzone.com/articles/mixins-python
} 


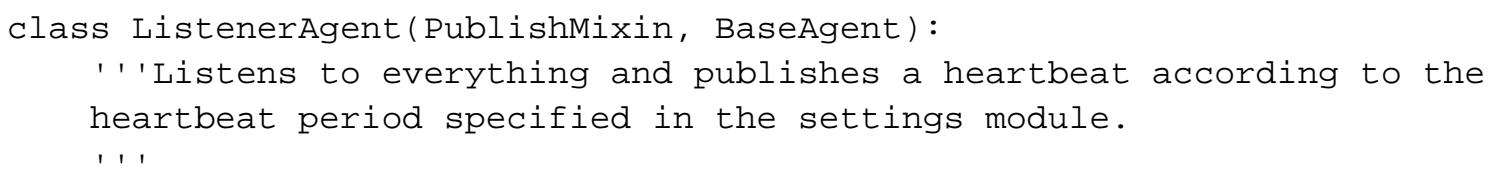

The Listener agent subscribes to all topics by using volttron/lite/agent/matching.py. This package contains decorators for simplifying subscriptions. The Listener agent uses match_all to receive all messages:

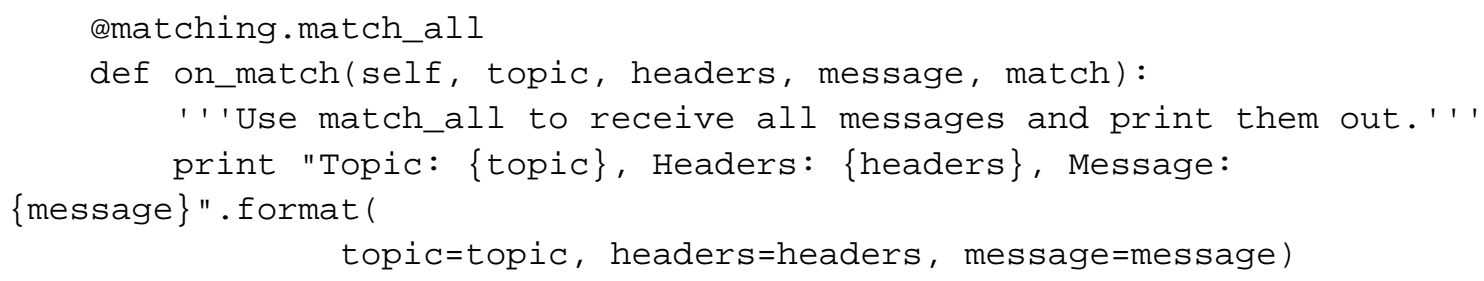

The Listener agent uses the @periodic decorator to execute the pubheartbeat method every HEARTBEAT_PERIOD seconds where HEARTBEAT_PERIOD is specified in the settings.py file. To publish, it creates a Header object to set the ContentType of the message, the time the event was created, and the ID of the agent sending it. This allows other agents to filter out messages of a certain type or from a certain agent. It also allows them to interpret the content appropriately. The message it then publishs out on the heartbeat topic.

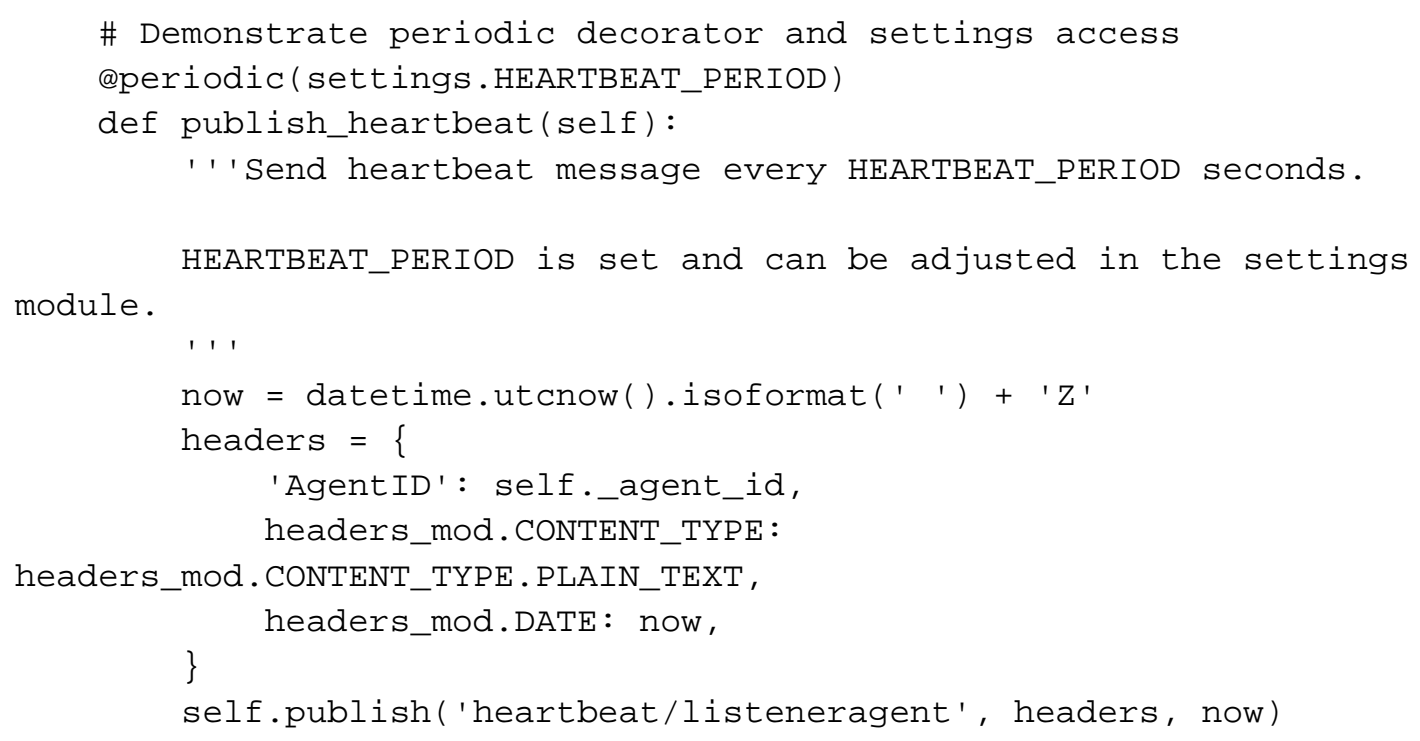

To see the Listener agent in action, please see Section 2.7.

\subsection{Agent Development in Eclipse}

The Eclipse IDE is not required for agent development, but it can be a powerful developmental tool. For those wishing to use it, download the IDE (integrated development environment) 
from: http://www.eclipse.org/ or through the Software Manager in Linux. The Transactional Network code is stored in a Git repository. There is a plugin available for Eclipse that makes development more convenient (note: you must have Git installed on the system and have built the project):

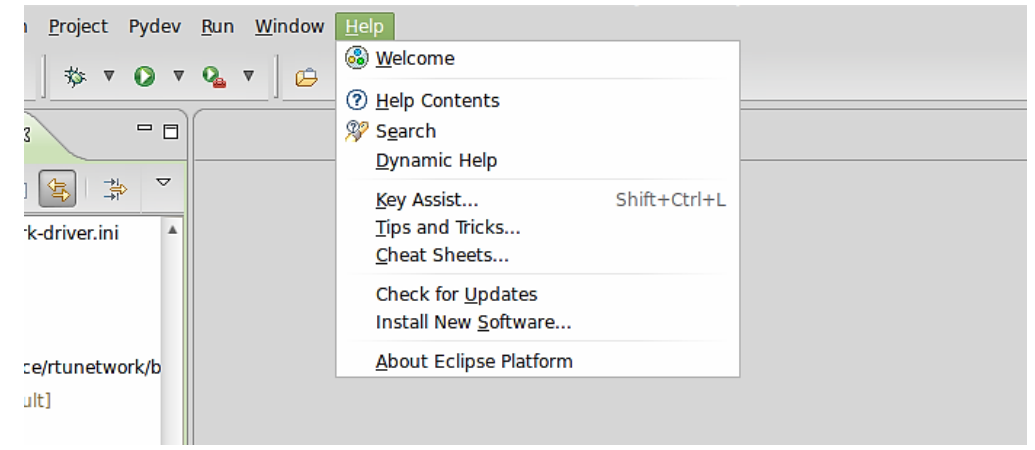

Figure 33: Installing Eclipse EGit Plug-in

- Help -> Install New Software

- $\quad$ Click on the "Add" button

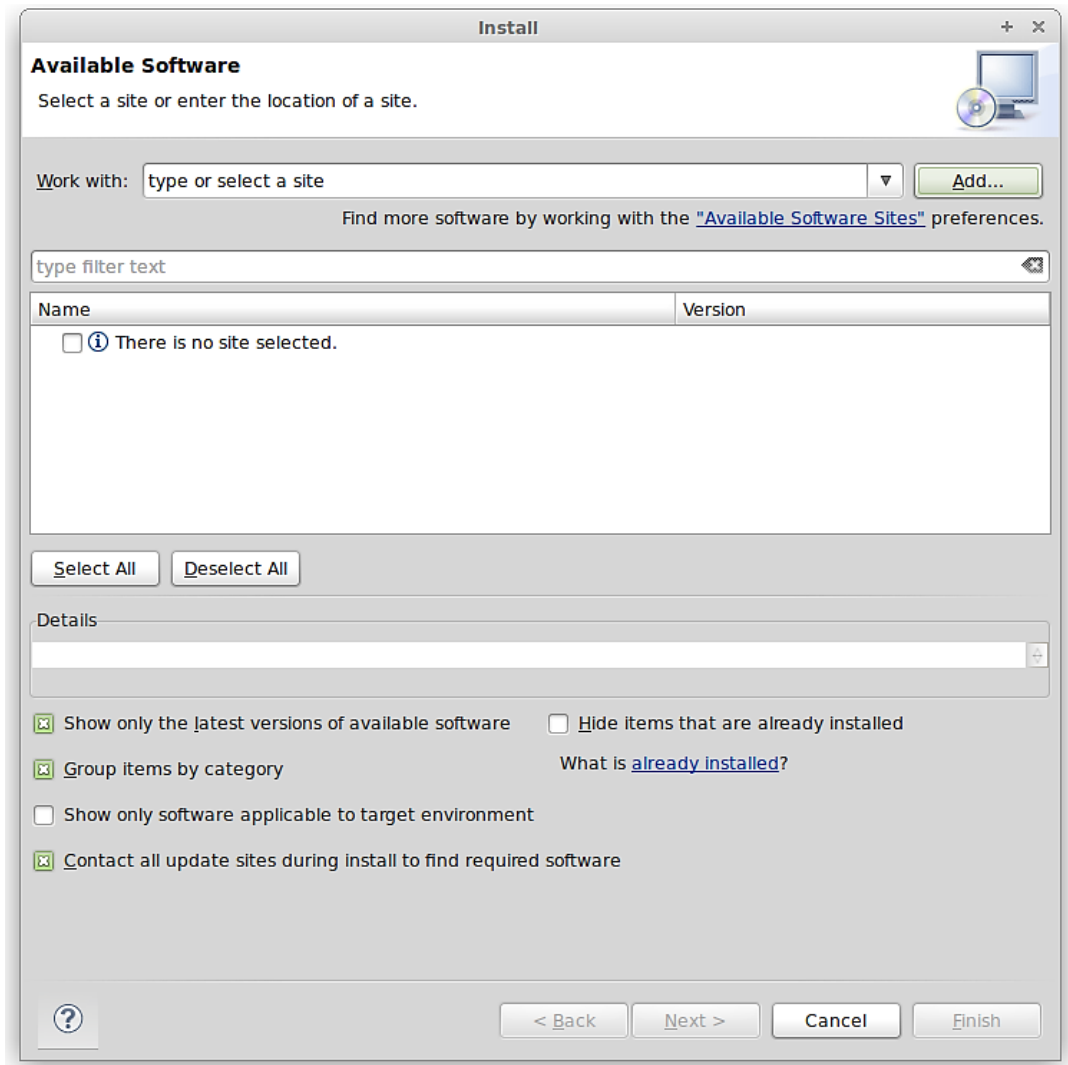

Figure 34: Installing Eclipse Egit Plug-in (continued)

- $\quad$ For name use: EGit

- For location: http://download.eclipse.org/egit/updates 


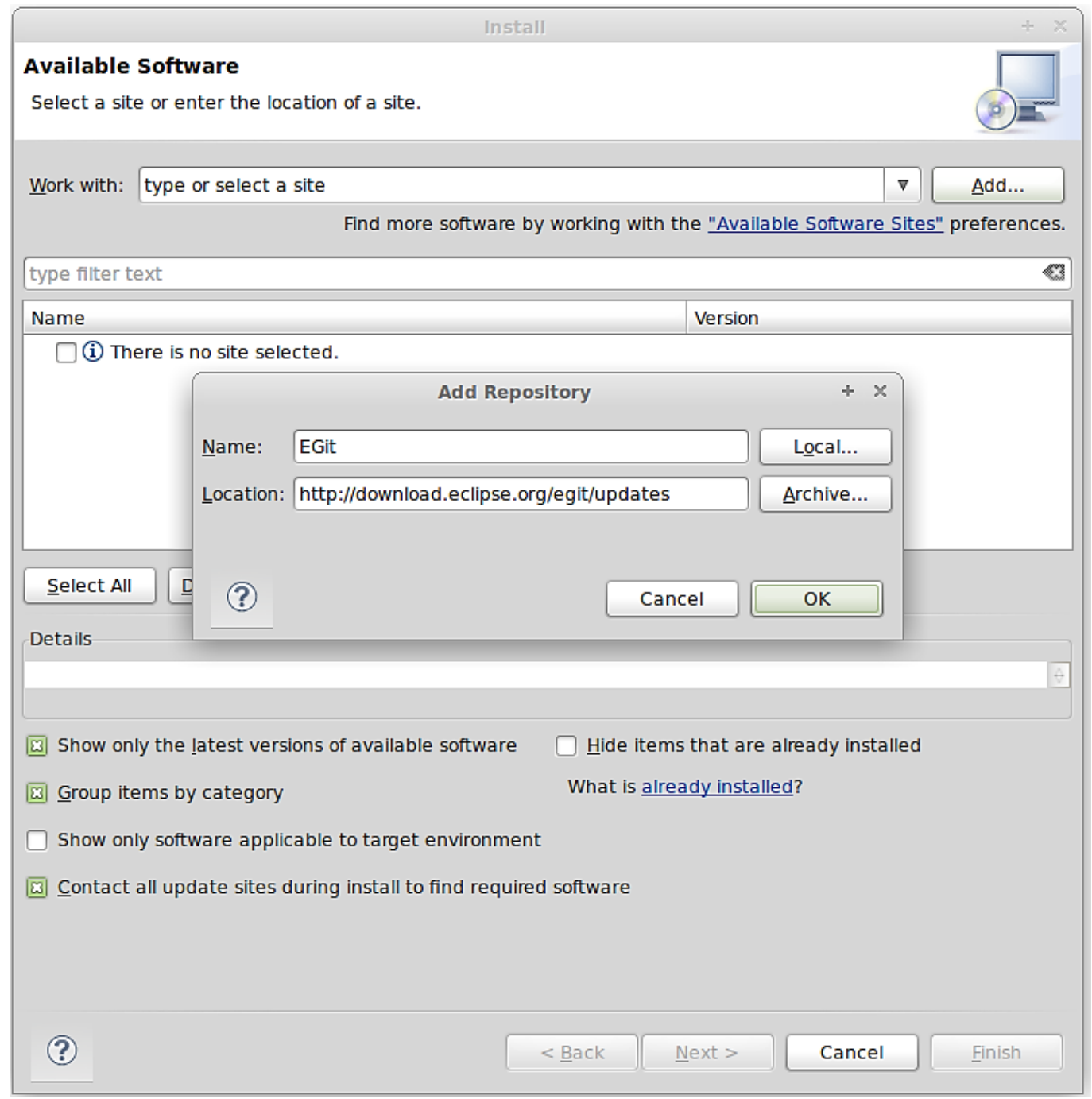

Figure 35: Installing Eclipse Egit Plug-in (continued)

- After hitting OK, check the Select All button

- $\quad$ Click through Next, Agree to Terms, then Finish

- Allow Eclipse to restart

After installing Eclipse, you must add the PyDev plugin to the environment. In Eclipse:

- Help -> Install New Software

- $\quad$ Click on the "Add" button 


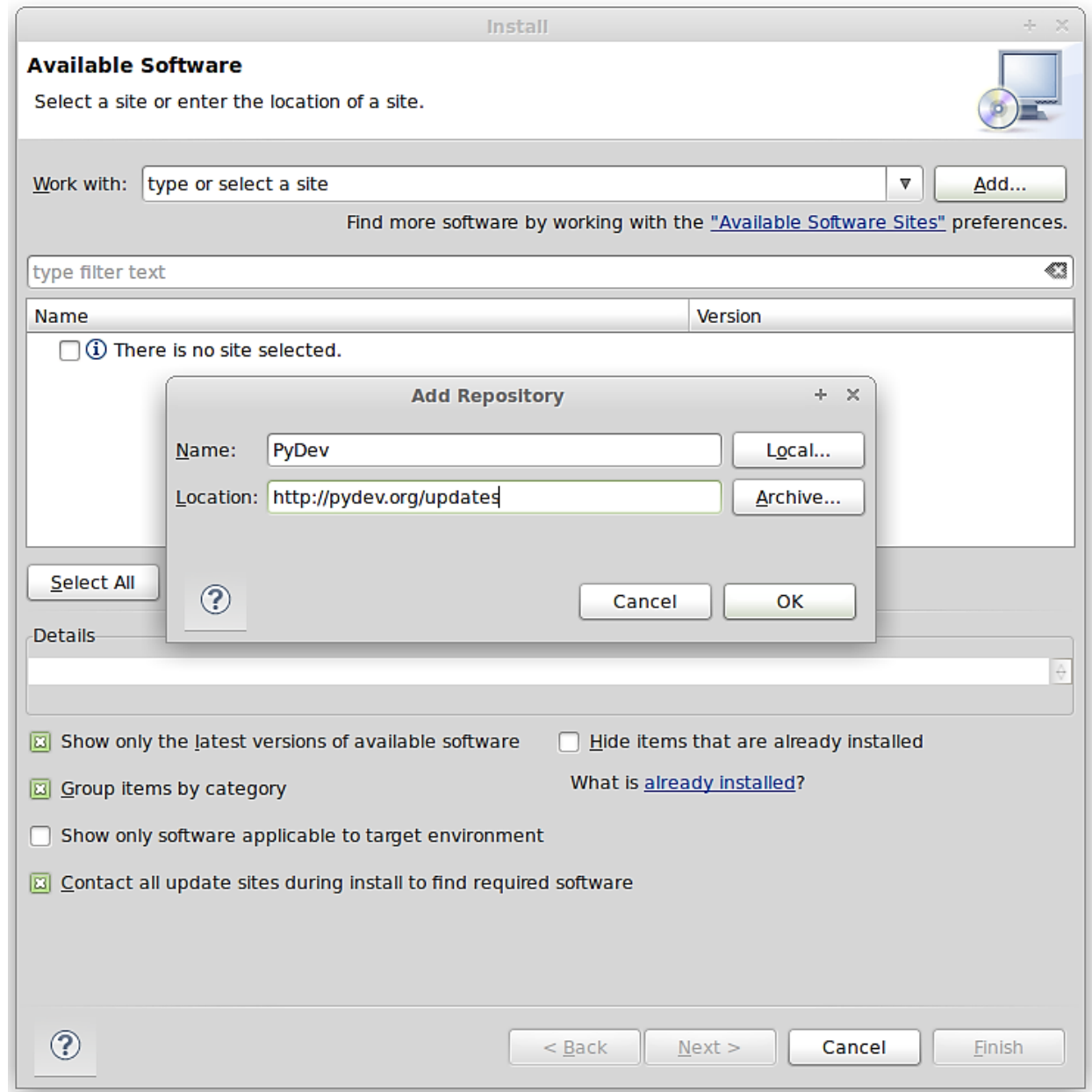

Figure 36: Installing Eclipse PyDev Plug-in

- For name use: PyDev

- For location: http://pydev.org/updates

- $\quad$ Click OK

- $\quad$ Check the box for PyDev

- Click through Next, Agree to Terms, Finish

- Allow Eclipse to restart

The project can now be checked out from the repository into Eclipse:

- $\quad$ Select File, then import as shown in Figure 37 


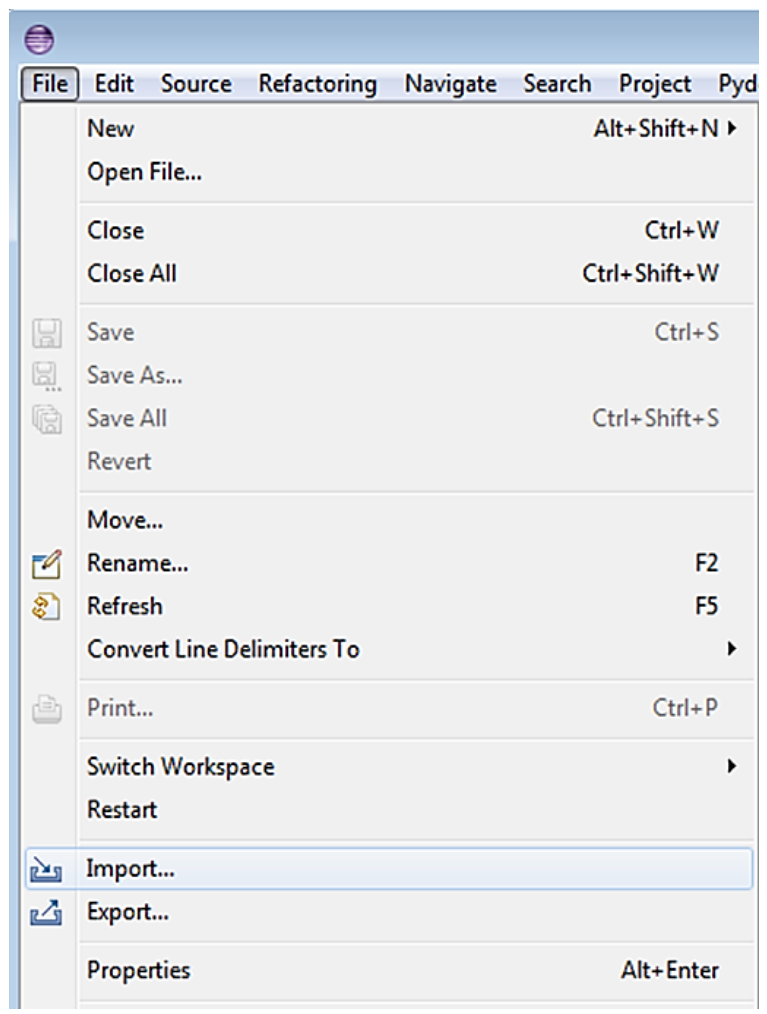

Figure 37: Checking Out Transactional Network with Eclipse

- Select Git -> Projects from Git, then click the Next button

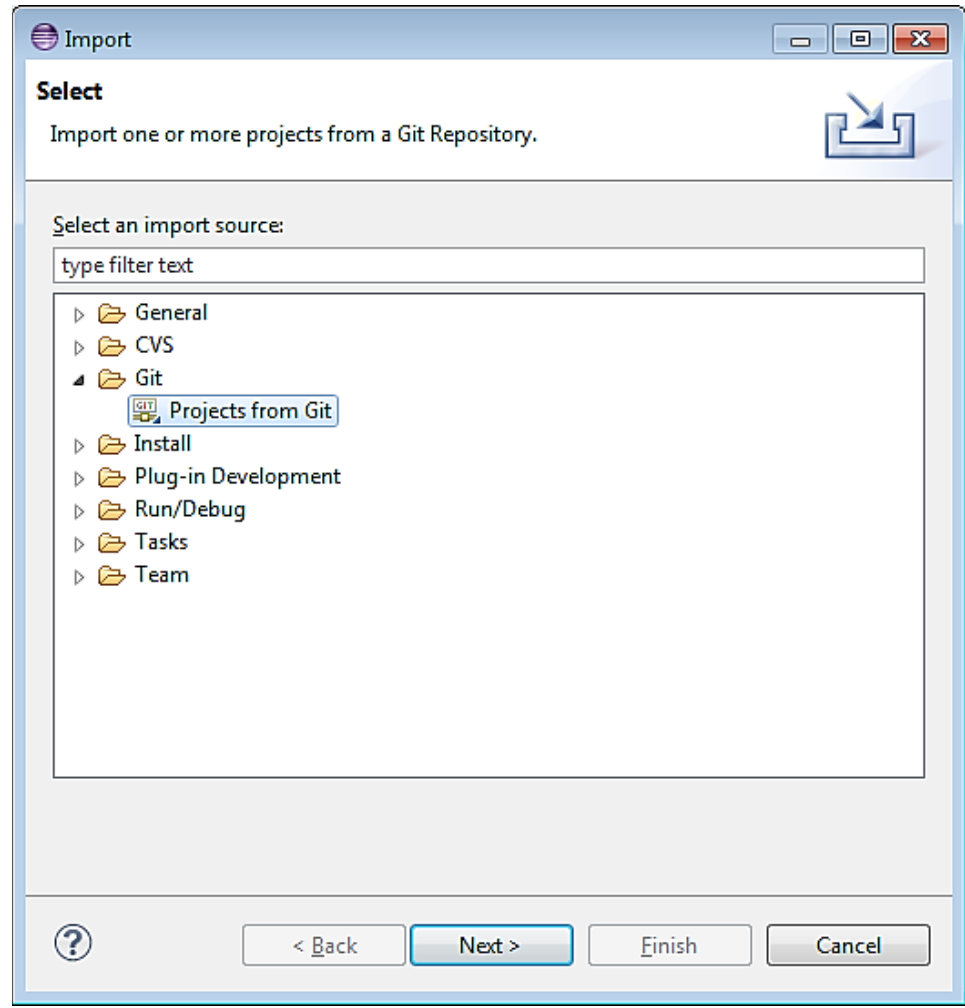

Figure 38: Checking Out Transactional Network with Eclipse (continued) 
- Fill in https://github.com/VOLTTRON/volttron.git for the URI, and use your github account login (github account username and password in the User and Password as shown in Figure 39)

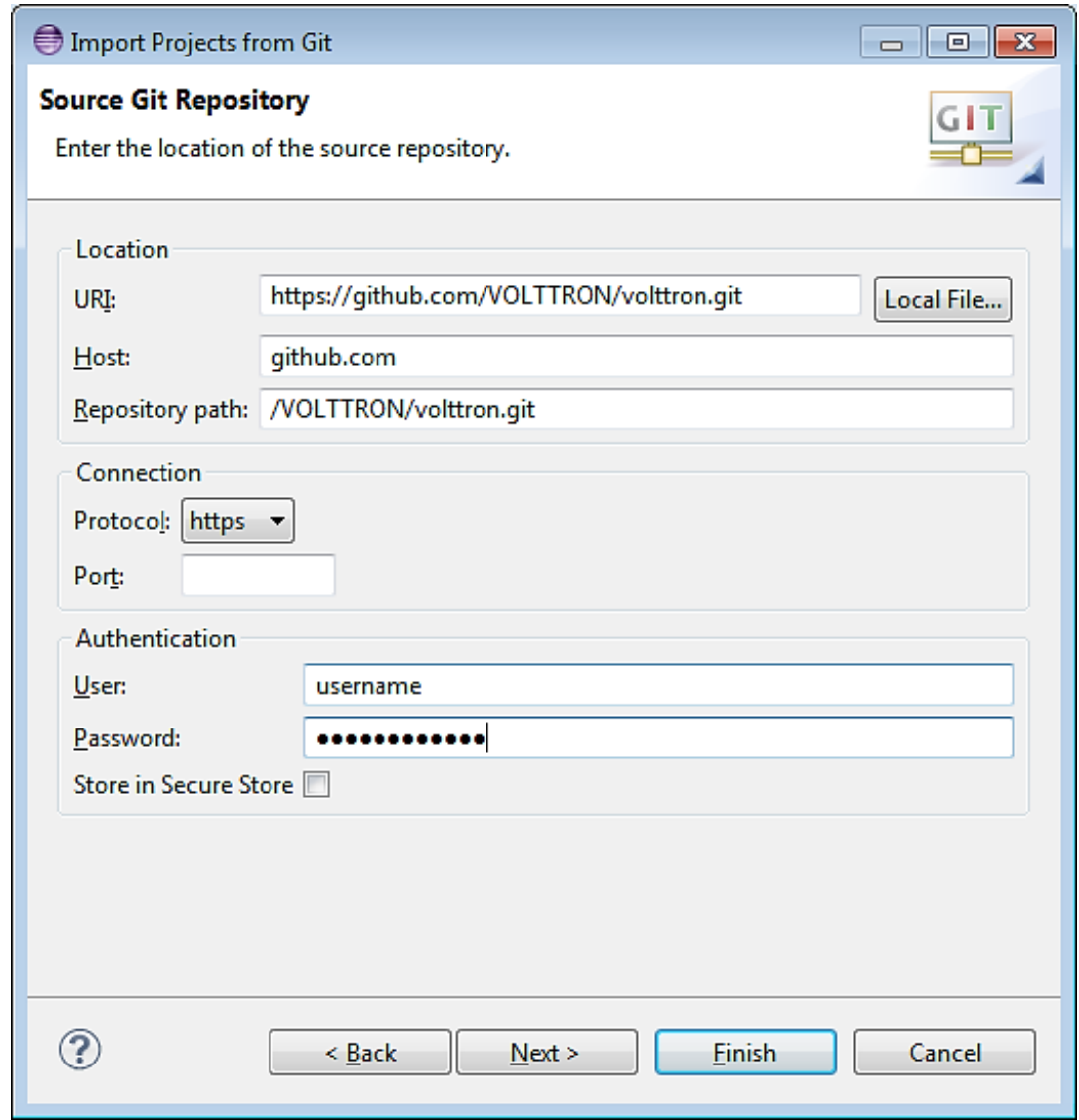

Figure 39: Checking Out Transactional Network with Eclipse (continued)

- $\quad$ Select the master branch (Figure 40) 


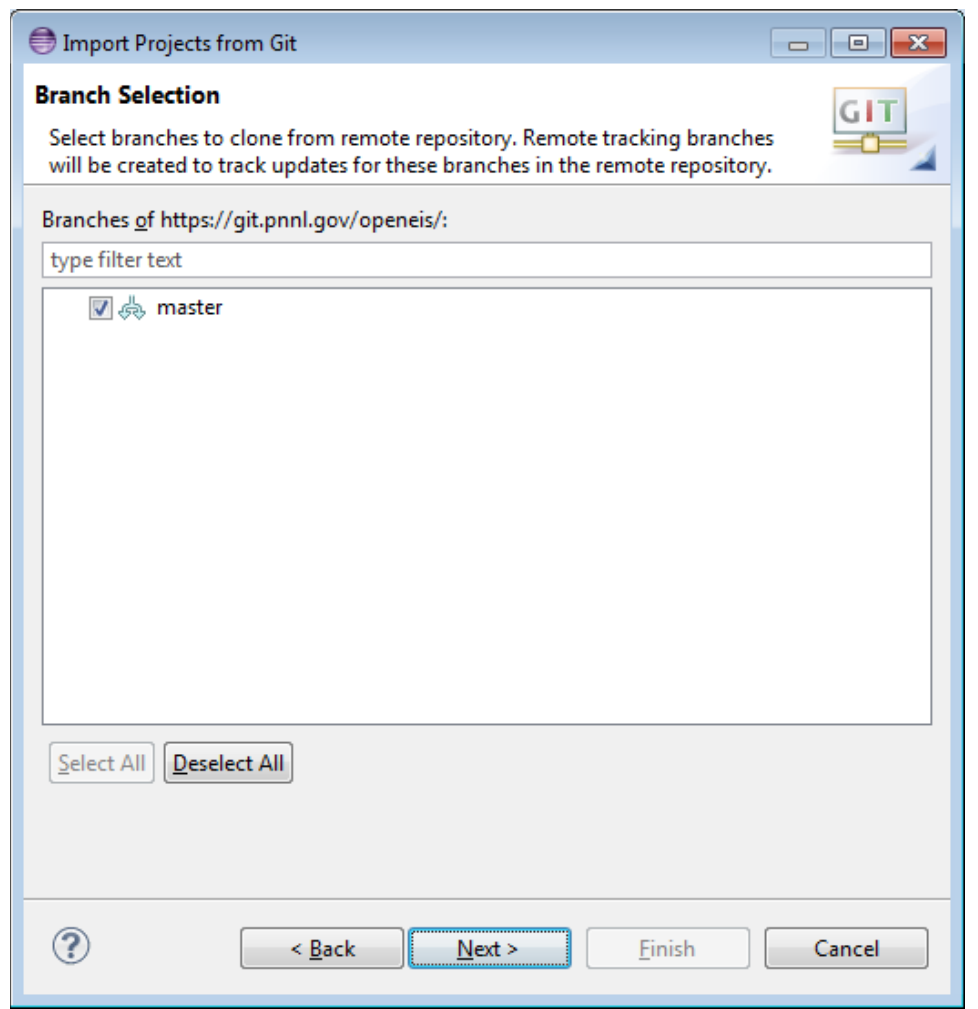

Figure 40: Checking Out Transactional Network with Eclipse (continued)

- $\quad$ Select a location to save the local repository (Figure 41)

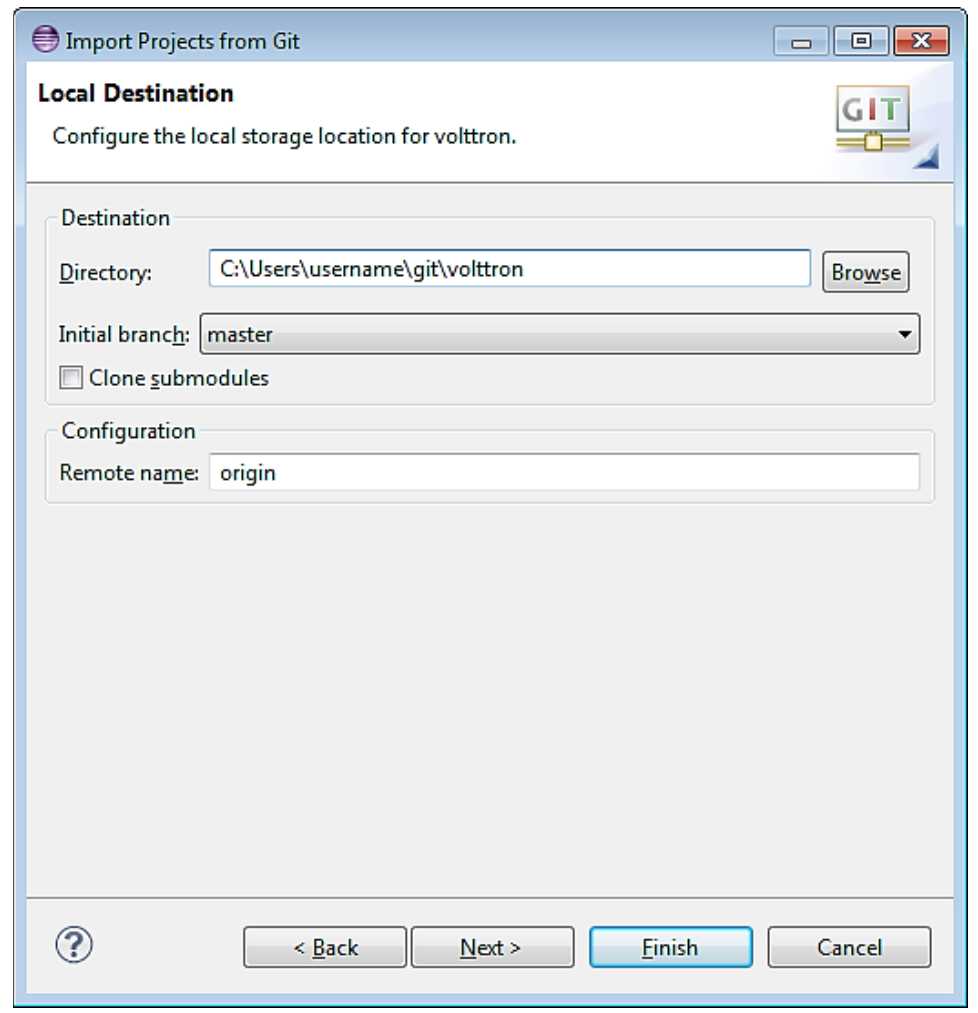

Figure 41: Checking Out Transactional Network with Eclipse (continued) 
- $\quad$ Select Import as general project, select Next, then select Finish (Figure 42)

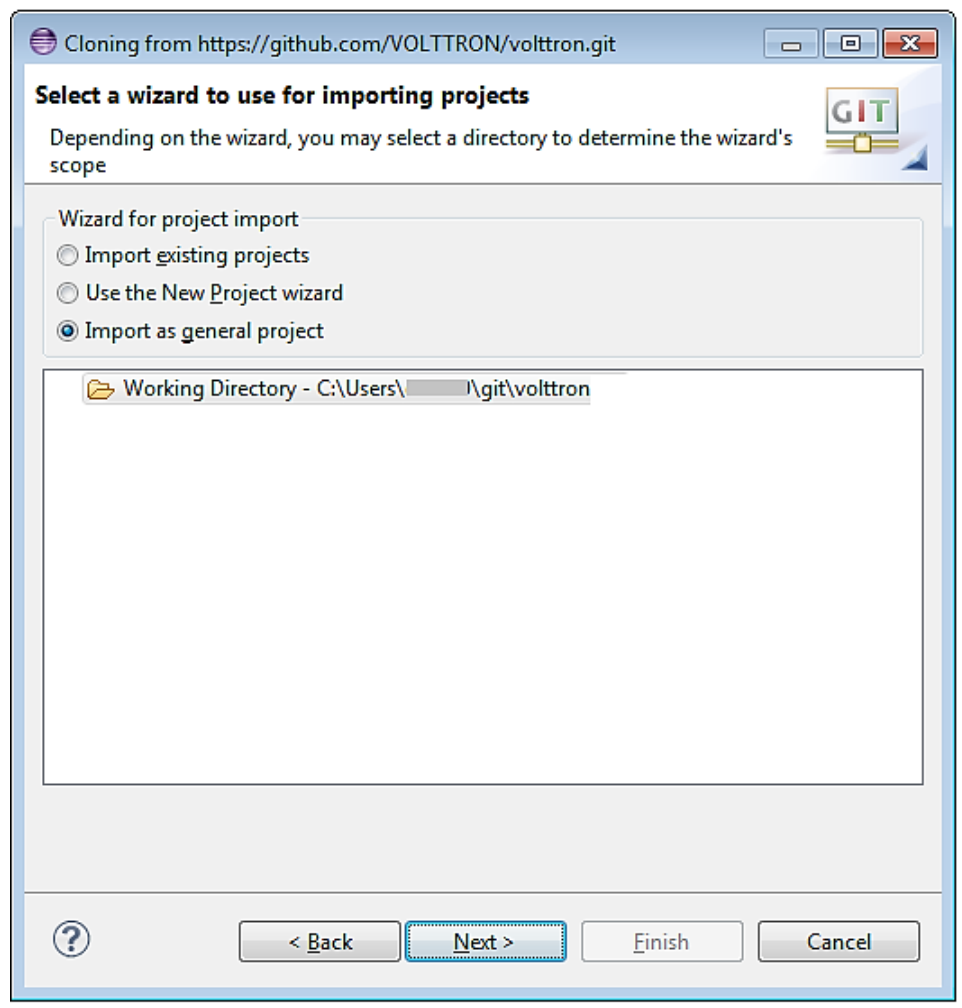

Figure 42: Checking Out Transactional Network With Eclipse (continued)

The project must now be built outside Eclipse. Please follow the directions in Section 2.6. After changing the file system outside Eclipse, right-click on the project name and select Refresh. PyDev must now be pointed at the correct version of Python:

- Window-> Preferences

- Expand PyDev

- $\quad$ Select Interpreter-Python

- Hit New

- Use Python as the Interpreter Name, and Hit Browse. In the bin directory for the volttron project select the file named Python, Then hit OK 


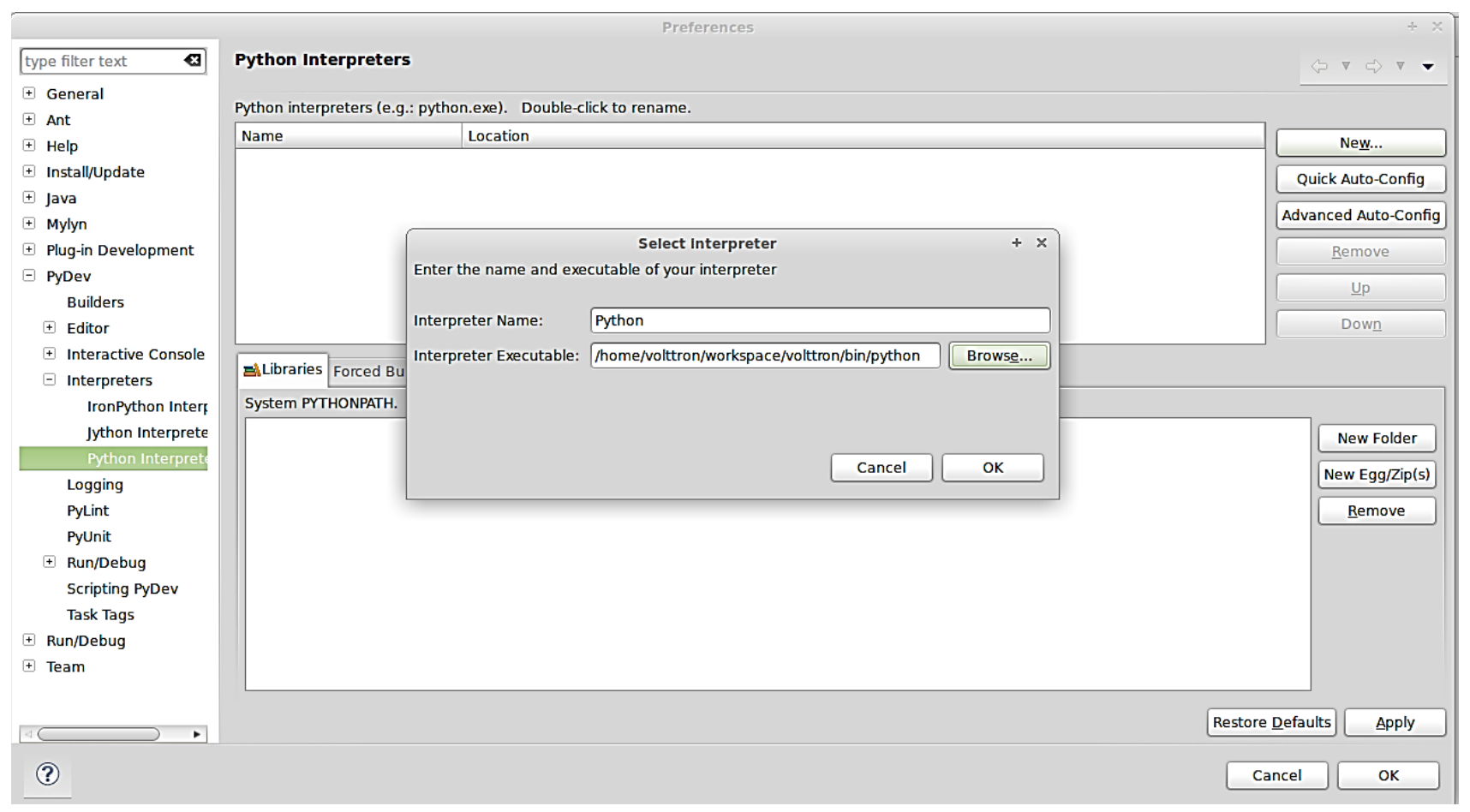

Figure 43: Configuring PyDev

- Select All, then Hit OK

You may need to redo this after a platform update and buildout.

- In the Project/PackageExplorer view on the left, right-click on the project, PyDev-> Set as PyDev Project

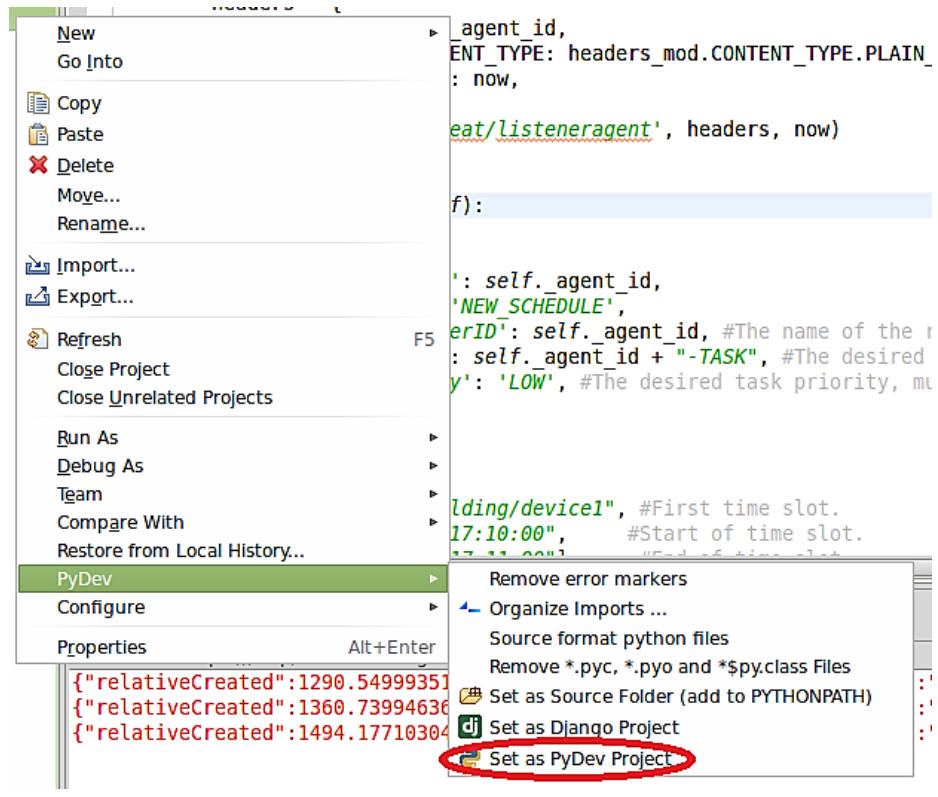

Figure 44: Setting as PyDev Project 
Switch to the PyDev perspective (if it has not already switched).

- Window -> Open Perspective -> PyDev or Window -> Open Perspective -> Other -> PyDev

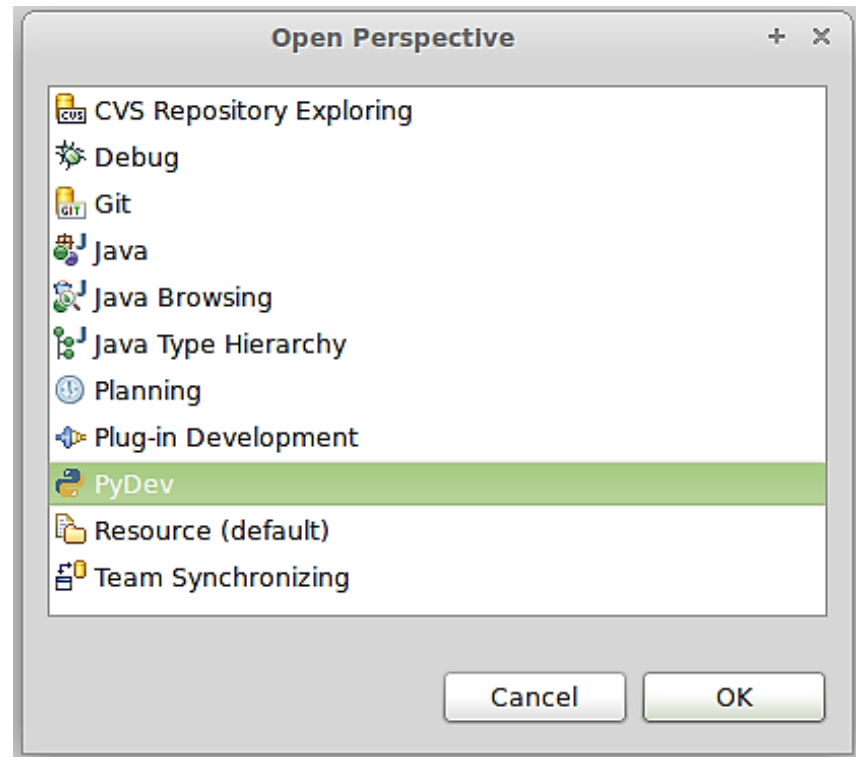

Figure 45: Setting PyDev Perspective in Eclipse

Eclipse should now be configured to use the project's environment.

\subsubsection{Setup a Run Configuration for the Platform}

The following steps will describe the process for configuring the platform within Eclispe:

- In the PyDev Package Explorer view, open the bin folder

- Right-click on volttron-lite and select Run As -> Python Run (this will create a run configuration but fail) 


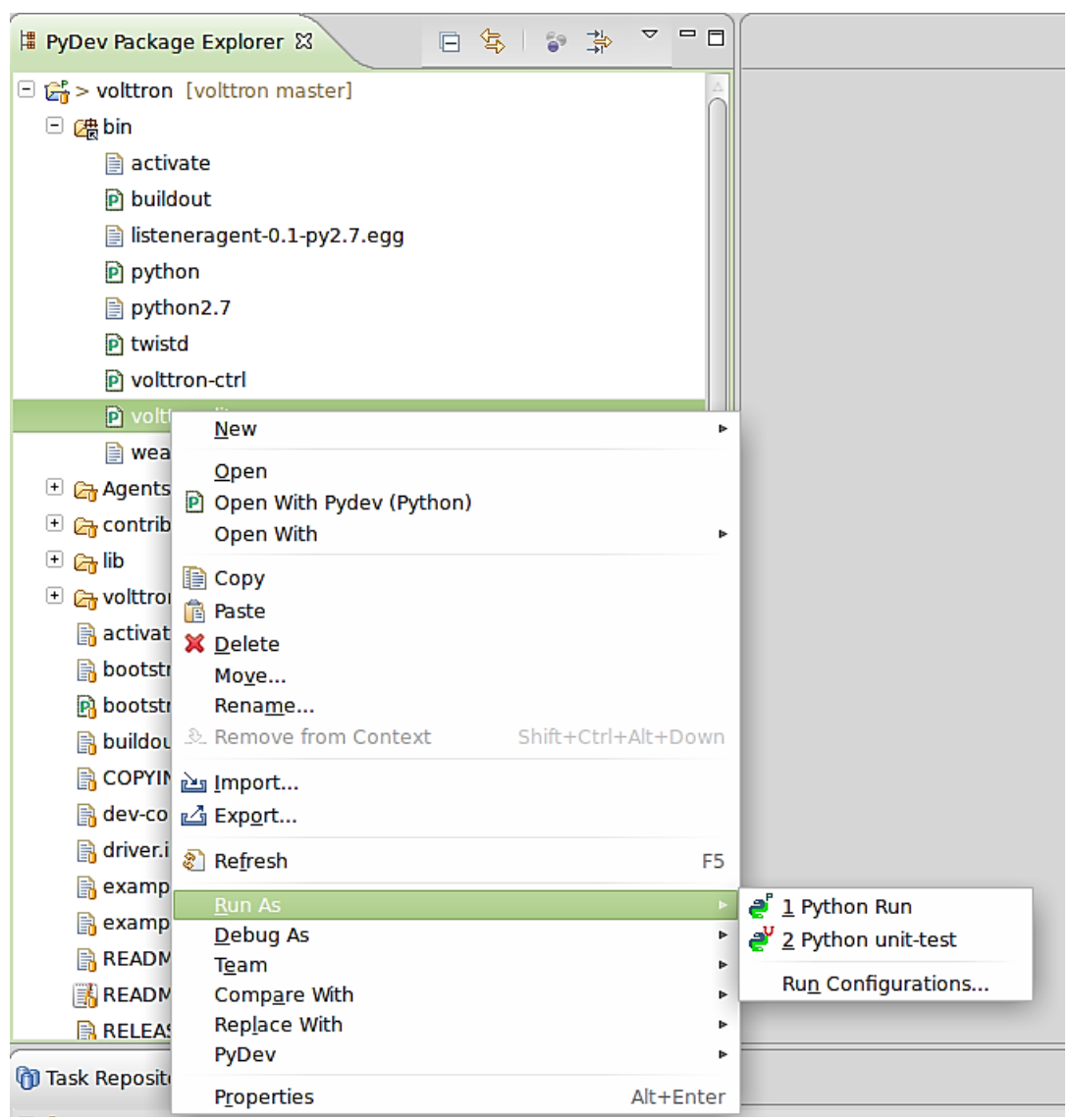

Figure 46: Running Platform Configuration in Eclipse

- On the menu bar, pick Run -> Run Configurations...

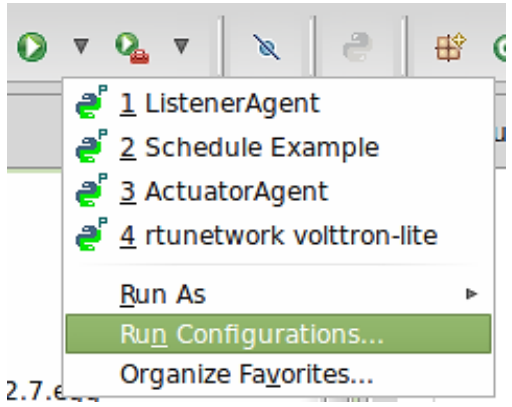

Figure 47: Running Platform Configuration in Eclipse (Continued)

- Under Python Run, pick "volttron volttron-lite"

- Click on the Argument tab

- Change Working Directory to Default 
- In the Program arguments box, add: "-c dev-config.ini - $v-v$ " This sets up running the platform in a development mode

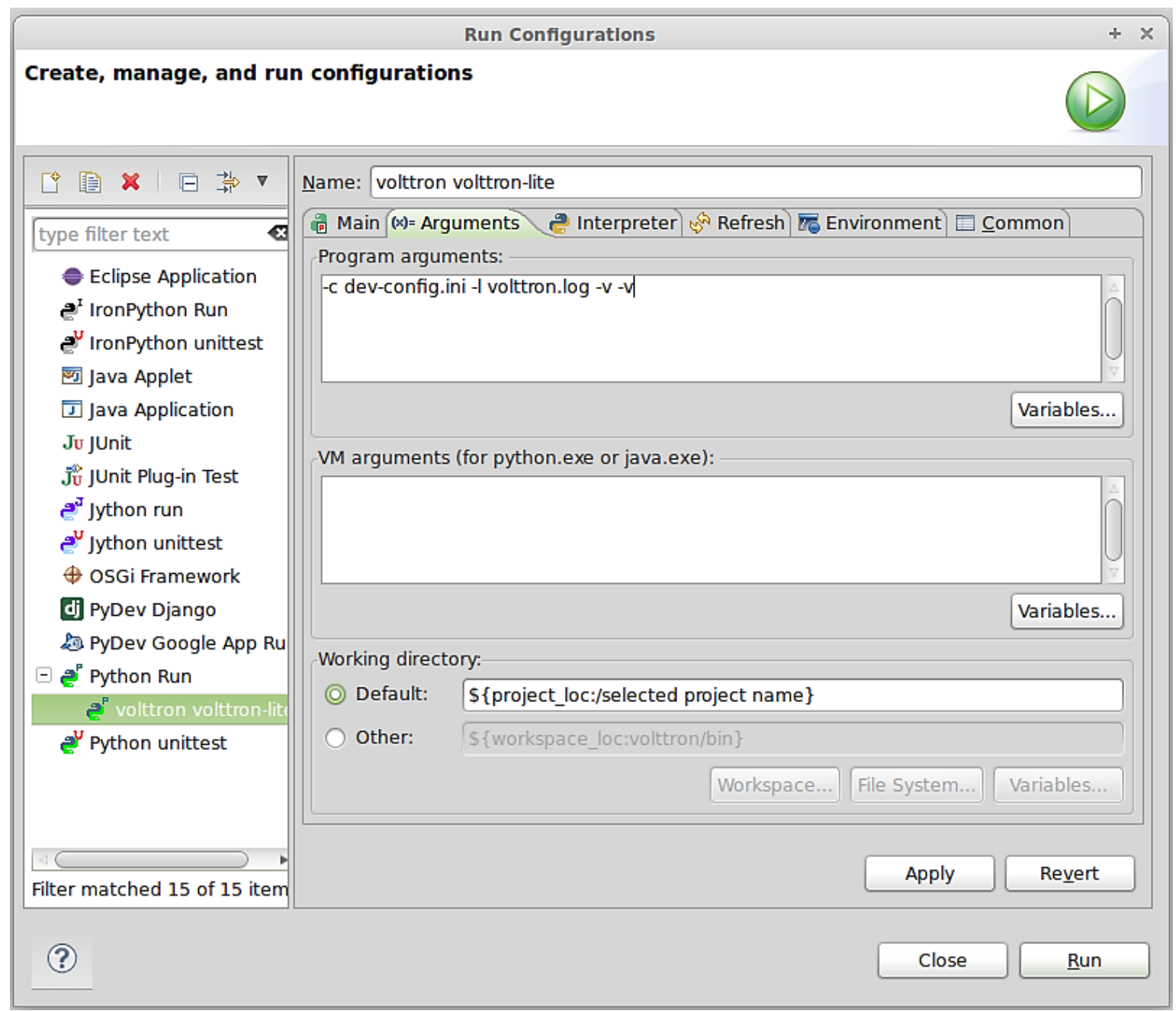

Figure 48: Running Platform Configuration in Eclipse (Continued)

- Click Run, this launches the platform. If the run does not succeed, click the all stop icon (two red boxes overlaid) on the console and then retry.

\subsubsection{Setup a run configuration for the Listener Agent}

The following steps will describe the process for configuring an agent within Eclispe:

- In the Package Explorer view, open Agents -> ListenerAgent --> listener

- Right-click on listeneragent.py and select Run As -> Python Run (this will create a run configuration but fail)

- $\quad$ On the menu bar, pick Run -> Run Configurations... 
- Under Python Run pick "volttron listeneragent.py"

- Click on the Argument tab

- Change Working Directory to Default

- In the Program arguments box, add:

o $\quad$ "--config Agents/ListenerAgent/listeneragent.launch.json --pub ipc:///tmp/volttron-liteagent-publish --sub ipc:///tmp/volttron-lite-agent-subscribe"

This configuration launches the agent with the dev settings the platform is using.

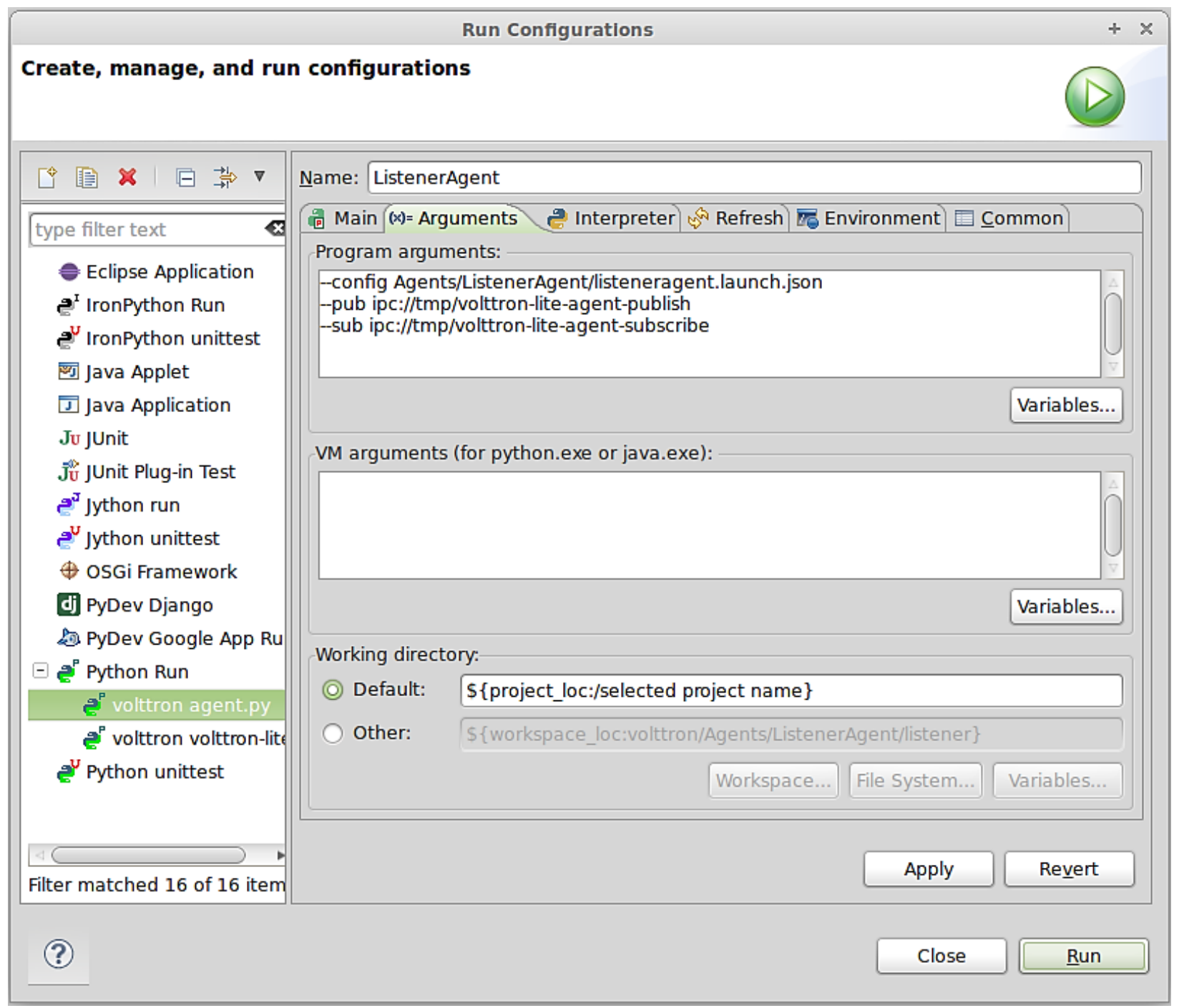

Figure 49: Configuring the ListenerAgent in Eclipse

- Click Run, this launches the agent 
You should see the agent start to publish and receive its own heartbeat message

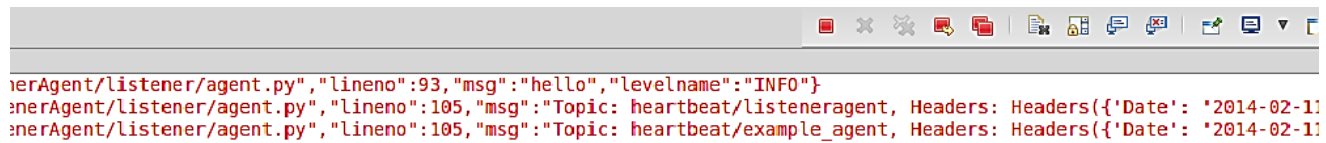

Figure 50: ListenerAgent Output on Eclipse Console

\subsection{Agent Creation Walkthrough}

It is recommended that developers look at the Listener agent before developing their own agent. That agent illustrates the basic functionality of an agent. The following example will demonstrate the steps for creating an agent.

\subsubsection{Agent Folder Setup}

Creating a folder within the workspace will help consolidate the code your agent will utilize.

- In the Agents directory, create a new folder TestAgent

- In TestAgent, create a new folder tester, this is the package where our Python code will be created

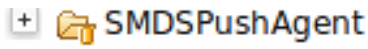 \\ $-\boxminus$ TestAgent $B$ tester \\ $+\Omega_{\square}$ WeatherAgent}

Figure 51: Creating an Agent Test Folder

\subsubsection{Create Agent Code}

The following steps describe the necessary agent files and modules.

- In tester, create a file called "__init_..py" which tells Python to treat this folder as a package

- In the tester package folder, create the file agent.py

- Create a class called TestAgent

- Import the packages and classes we will need:

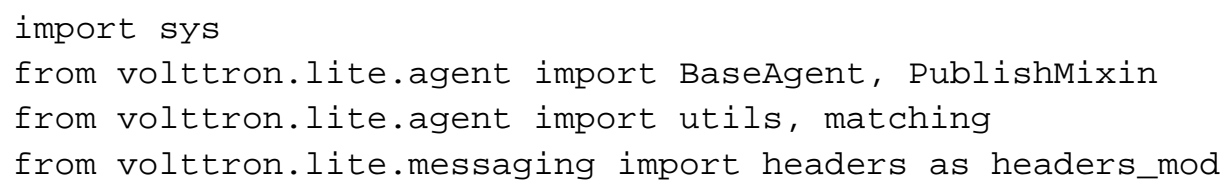


This agent will inherit features from the BaseAgent extending the agent's default functionality. Because we want to publish, we will use the PublishMixin. Mixins should be put first in the class definition.

class TestAgent(PublishMixin, BaseAgent):

BaseAgent has several methods we could overwrite if we needed to application specific actions (init, setup, etc.). For purposes of this demonstration we will use default behavior.

\subsubsection{Setting up a Subscription}

We will set our agent up to listen to heartbeat messages (published by Listener agent). Using the matching package, we declare we want to match all topics that start with "heartbeat/listeneragent". This will give us all heartbeat messages from all listener agents but no others.

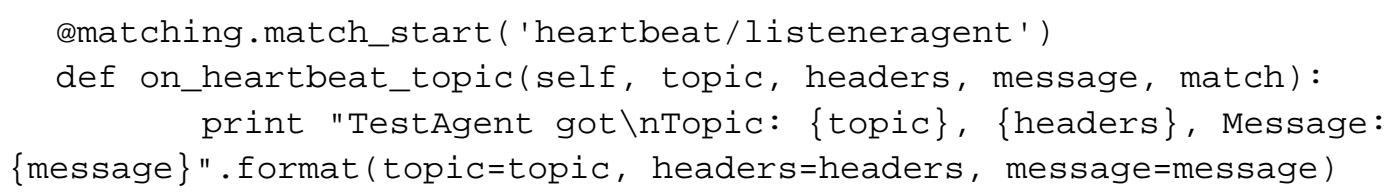

\subsubsection{Argument Parsing and Main}

The test agent will need to be able to parse arguments being passed on the command line by the agent launcher. Use the utils.default_main method to handle argument parsing and other default behavior.

- Create a main method that can be called by the launcher.

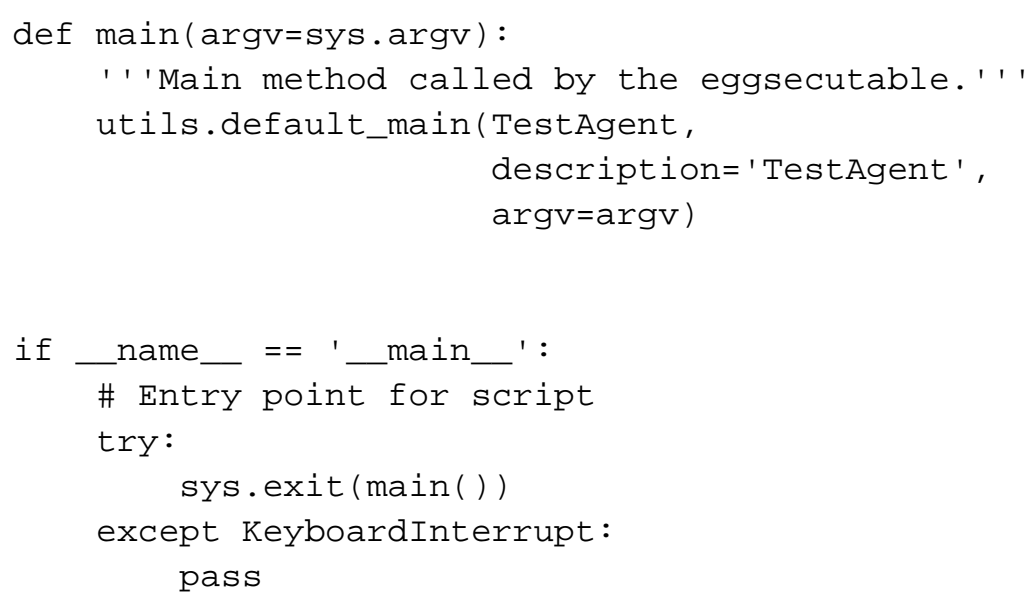

\subsection{Create Support Files for Test Agent}

VOLTTRON agents need configuration files for packaging, configuration, and launching. The "setup.py" file details the naming and Python package information. The launch configuration file is a JSON formatted text file used by the platform to launch instances of the agent.

\subsubsection{Packaging Configuration}

In the TestAgent folder, create a file called "setup.py" (or copy the setup.py in ListenerAgent), which we will use to create an eggsecutable. This file sets up the name, version, required packages, method to execute, etc. for the agent. The packaging process will also use this information to name the resulting file.

from setuptools import setup, find_packages 


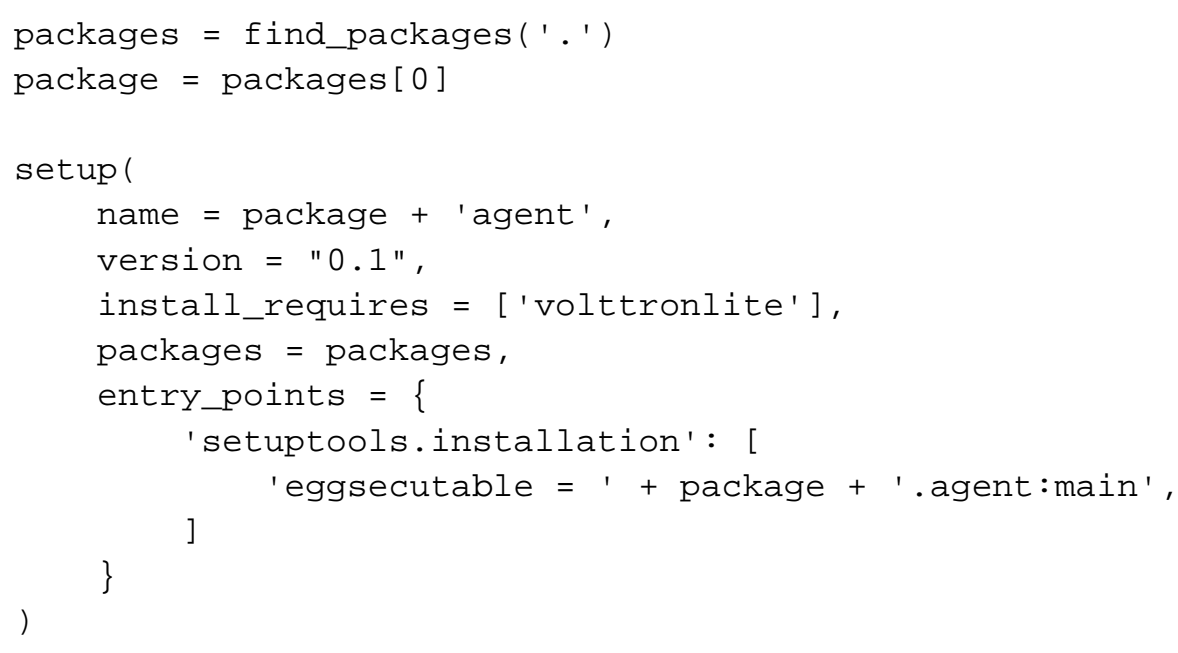

\subsubsection{Launch Configuration}

In TestAgent, create a file called "testagent.launch.json". This is the file the platform will use to launch the agent. It can also contain configuration information for the agent.

\{

"agent":

"exec": "testeragent-0.1-py2.7.egg --config \"\%c\" --sub \"\%s\" - - pub \"\%p\""

\},

"agentid": "Test1",

"message": "hello"

\}

\subsubsection{Packaging Agent}

The agent code must now be packaged for use by the platform. The build-agent.sh script will build the eggsecutable package using the setup.py file we defined earlier.

- On the command line, from the volttron directory:

0 volttron/scripts/build-agent.sh TestAgent

This creates an egg file in the Agents directory, which, along with the launch configuration file, would be sent to a deployed platform for installation. For local testing, you may need to change permissions on the file:

- $\quad$ chmod +x Agents/testeragent-0.1-py2.7.egg

\subsubsection{Testing the Agent}

From the Command Line in the project directory:

- Start the platform (if it isn’t already) by running:

\section{o bin/volttron-lite -c dev-config.ini -l volttron.log -v -v}

- Install the eggsecutable into the platform: 


\section{o bin/volttron-ctrl install-executable Agents/testeragent-.1-py2.7.egg}

- $\quad$ Load the agent configuration file:

\section{o bin/volttron-ctrl load-agent Agents/TestAgent/testagent.launch.json"}

- Launch the agent by running

\section{o bin/volttron-ctrl start_agent testagent.launch.json}

- $\quad$ Launch the Listener agent, as in Section 2.7. Check that both agents are running:

\section{$0 \quad$ bin/volttron-ctrl list-agents}

- $\quad$ Check the log file for messages:
o tail volttron.log

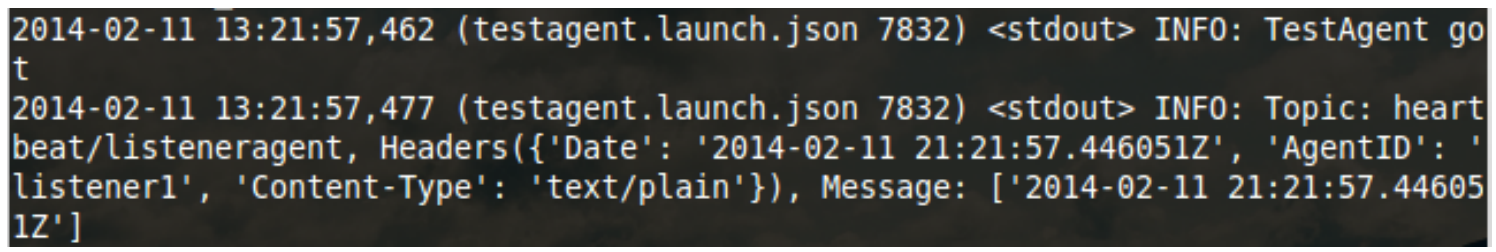

Figure 52: TestAgent Output in "volttron.log"

\subsubsection{Reloading the Agent}

After making changes to the code, the agent egg must be rebuilt and reloaded into the platform. If the configuration file is modified, it also must be reloaded and redeployed.

From the command line in the project directory, do the following commands. These assume that the TestAgent is already installed and running. It stops the agent, unloads the eggsecutable and configuration file, reloads the new ones, and starts the agent back up.

- $\quad$ Stop the agent if it is running:

o bin/volttron-ctrl stop-agent testagent.launch.json

- Rebuild the agent:

o volttron/scripts/build-agent.sh TestAgent

- Change permissions on the eggsecutable file:

o chmod +x Agents/testeragent-0.1-py2.7.egg

- Remove the previously installed eggsecutable:

$0 \quad$ bin/volttron-ctrl remove-executable testeragent-0.1-py2.7.egg

- Install the new eggsecutable:

o bin/volttron-ctrl install-executable Agents/testeragent-0.1-py2.7.egg 
- Unload the previous agent configuration:

\section{o bin/volttron-ctrl unload-agent testagent.launch.json}

- $\quad$ Load the new agent configuration:

\section{o bin/volttron-ctrl load-agent Agents/TestAgent/testagent.launch.json}

- $\quad$ Start the agent:

\section{$0 \quad$ bin/volttron-ctrl start-agent testagent.launch.json}

\subsubsection{In Eclipse}

If you are working in Eclipse, create a run configuration for TestAgent based on the Listener agent configuration in EclipseDevEnvironment (Section 4.3).

- $\quad$ Launch the platform

- Launch the TestAgent

- Launch the Listener agent

- TestAgent should start receiving the heartbeats from Listener agent and the following should be displayed in the console:

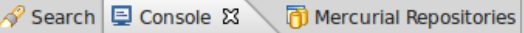

ipc:///tmp/volttron-lite-agent-subscribe

Topic: heartbeat/listeneragent, Headers(\{u'Date': u'2014-02-14 00:22:00.067964Z', u'AgentID': u'listener1', u'Content-Type': u'text TestAgent got

Topic: heartbeat/listeneragent, Headers(\{u'Date': u'2014-02-14 00:22:05.069604Z', u'AgentID': u'listener1', u'Content-Type': u'text TestAgent got

Topic: heartbeat/listeneragent, Headers(\{u'Date' : u'2014-02-14 00:22:10.067992Z', u'AgentID': u'listener1', u'Content-Type': u'text TestAgent got

Figure 53: Console Output for TestAgent
}

\subsection{Additional Features}

Additional code can be added to the TestAgent to utilize additional services in the platform. The following sections will show how to use the Weather agent and the Scheduler agent. After making modifications to the TestAgent to use these features, follow the instructions in Section 4.6.3.

\subsubsection{Subscribing to Weather Data}

This agent can be modified to listen to weather data from the Weather agent by adding the following method. This will subscribe the agent to the temperature topic. For the full list of topics available, please see: https://github.com/VOLTTRON/volttron/wiki/WeatherAgentTopics

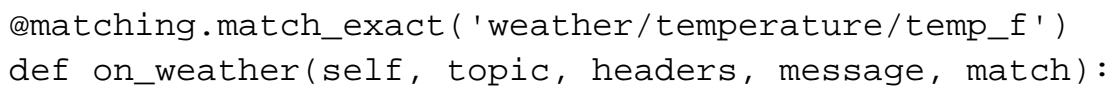


print "TestAgent got weather\nTopic: \{topic\}, \{headers\}, Message: \{message\}".format(topic=topic, headers=headers, message $=$ message $)$

The platform log file should show:

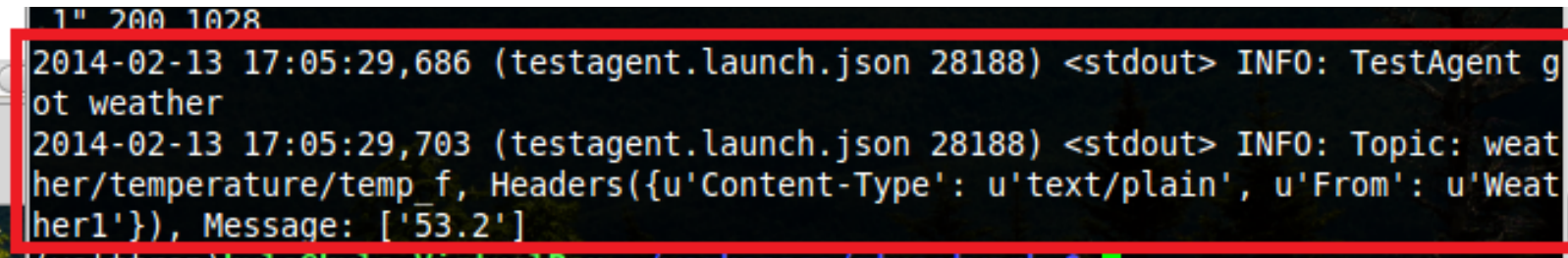

Figure 54: TestAgent Output when Subscribing to Weather Topic

\subsubsection{Utilizing the Scheduler Agent}

The TestAgent can be modified to publish a schedule to the Actuator agent by reserving time on fake devices. Modify the following code to include current time ranges and include a call to the publish schedule method in setup. The following example will post a simple schedule. For more detailed information on device scheduling, please see: https://github.com/VOLTTRON/volttron/wiki/ActuatorAgent

Ensure the Actuator agent is running as per Section 2.10. Then, import the messaging package so TestAgent can use the topics for the Scheduler:

from volttron.lite.messaging import topics

Then, create __init__ and setup methods so it can pull the agent id from its config file. These methods will override the BaseAgent methods, so we should call them with "super" so the default actions still happen. Then call the publish_schedule command, which is detailed later.

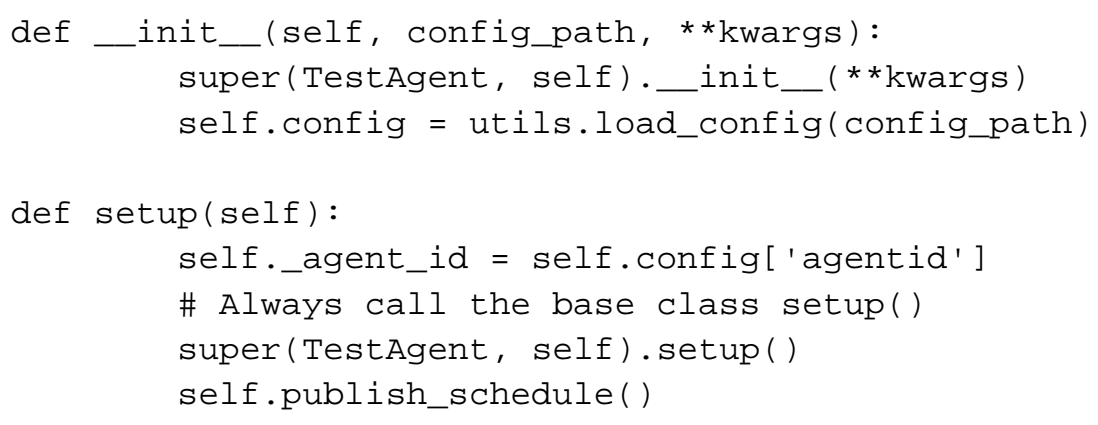

This calls the following method, which pushes a schedule request message to the Actuator agent (Update the schedule with appropriate times):

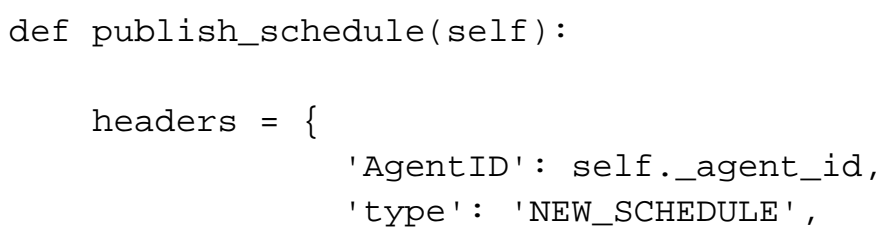




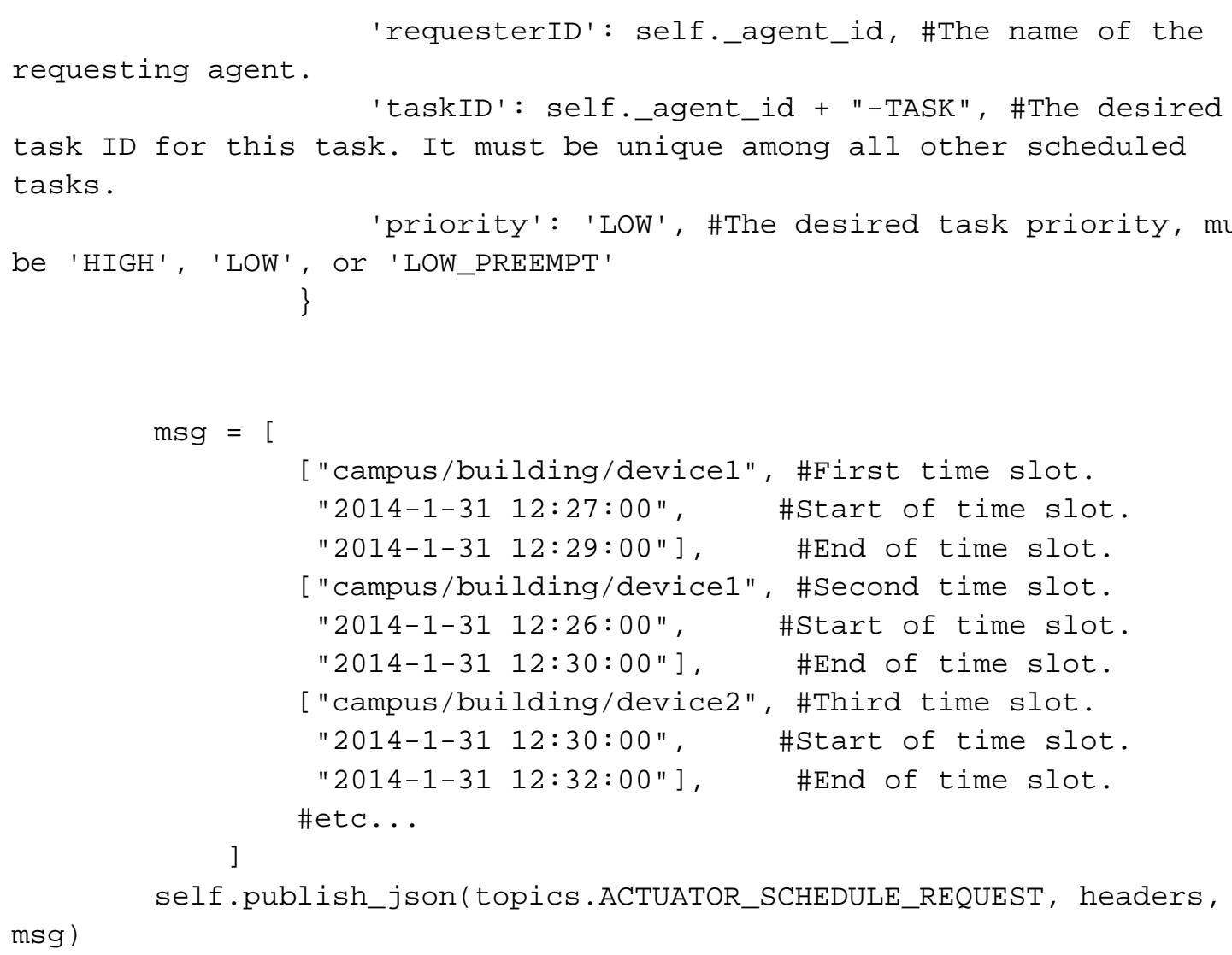

The agent can listen to the results of its request and get schedule announcements on the following topic:

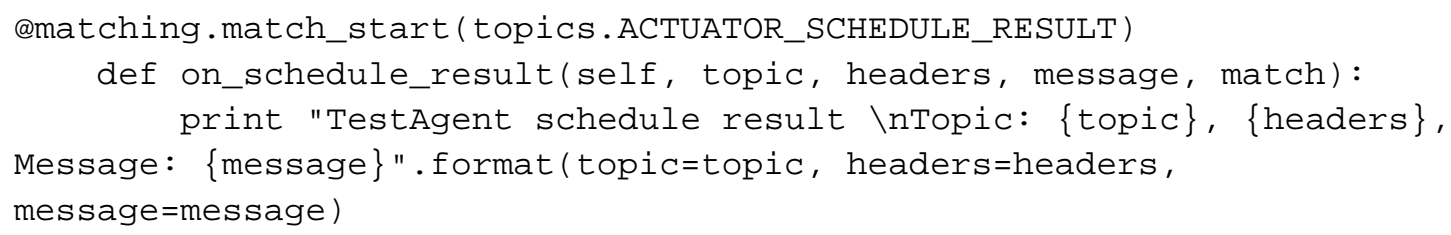

\subsubsection{Full TestAgent Code}

The following is the full TestAgent code built in the previous steps:

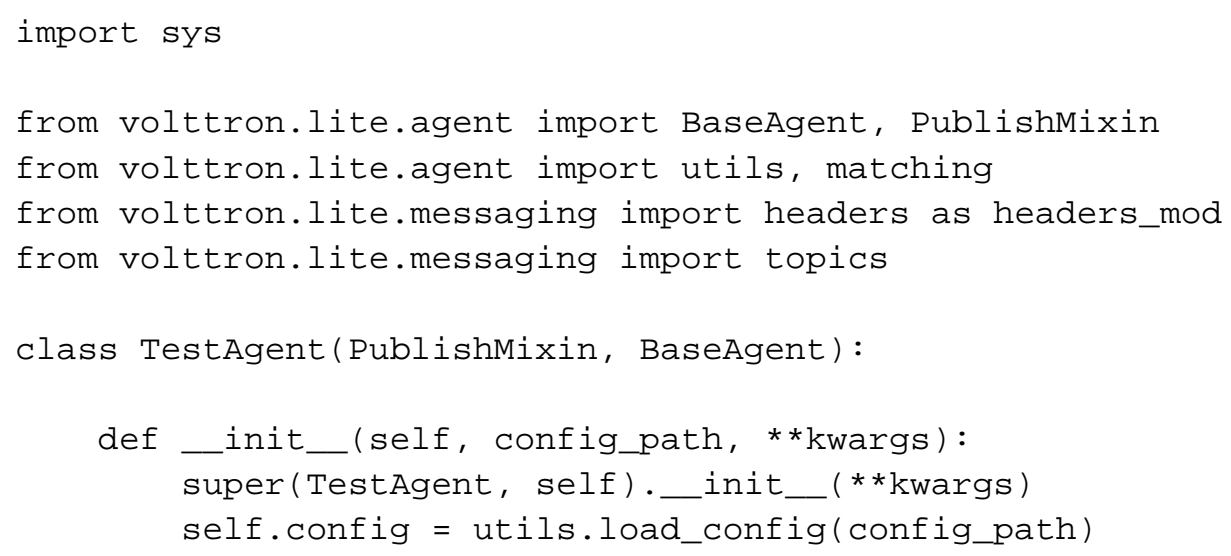




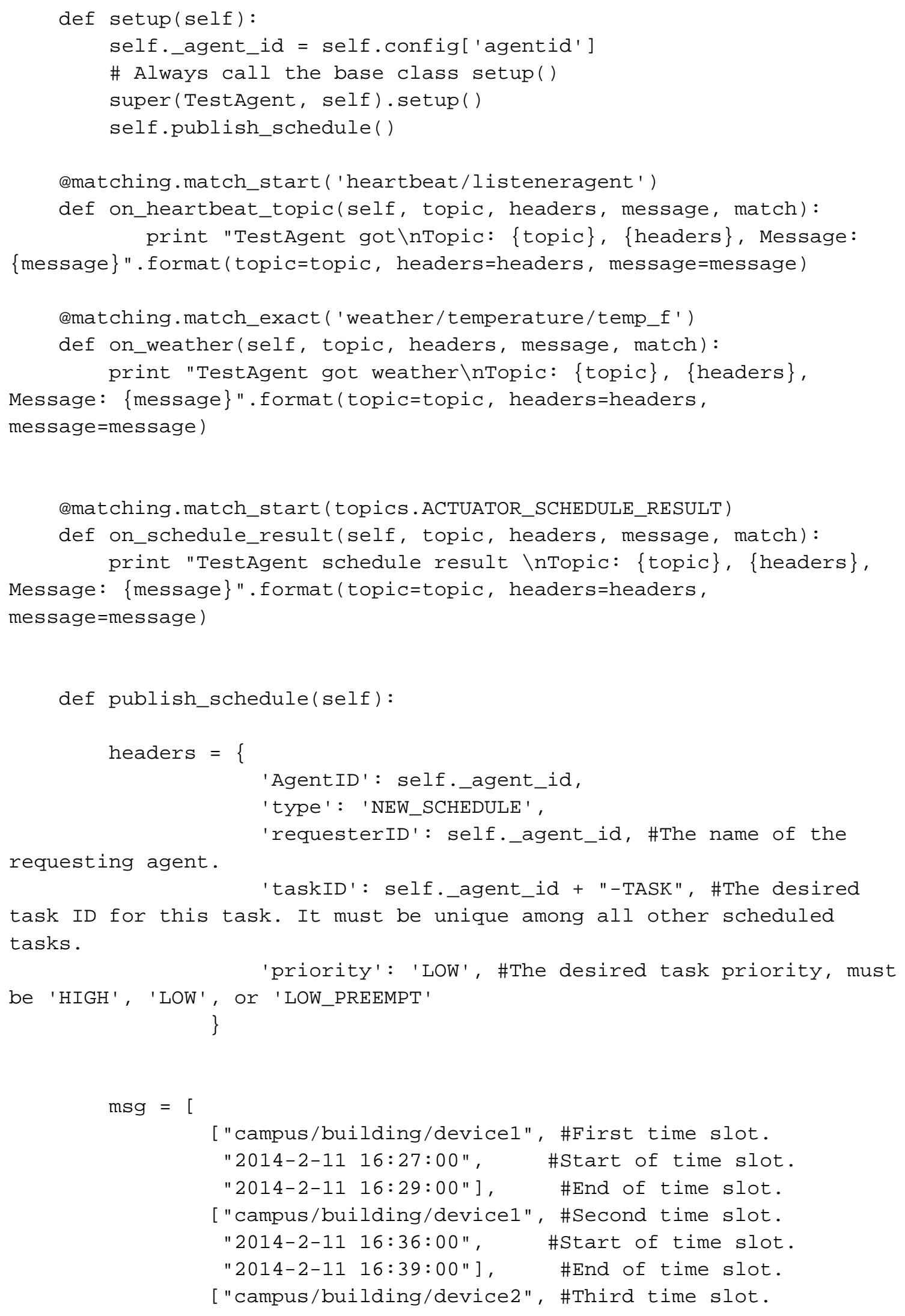




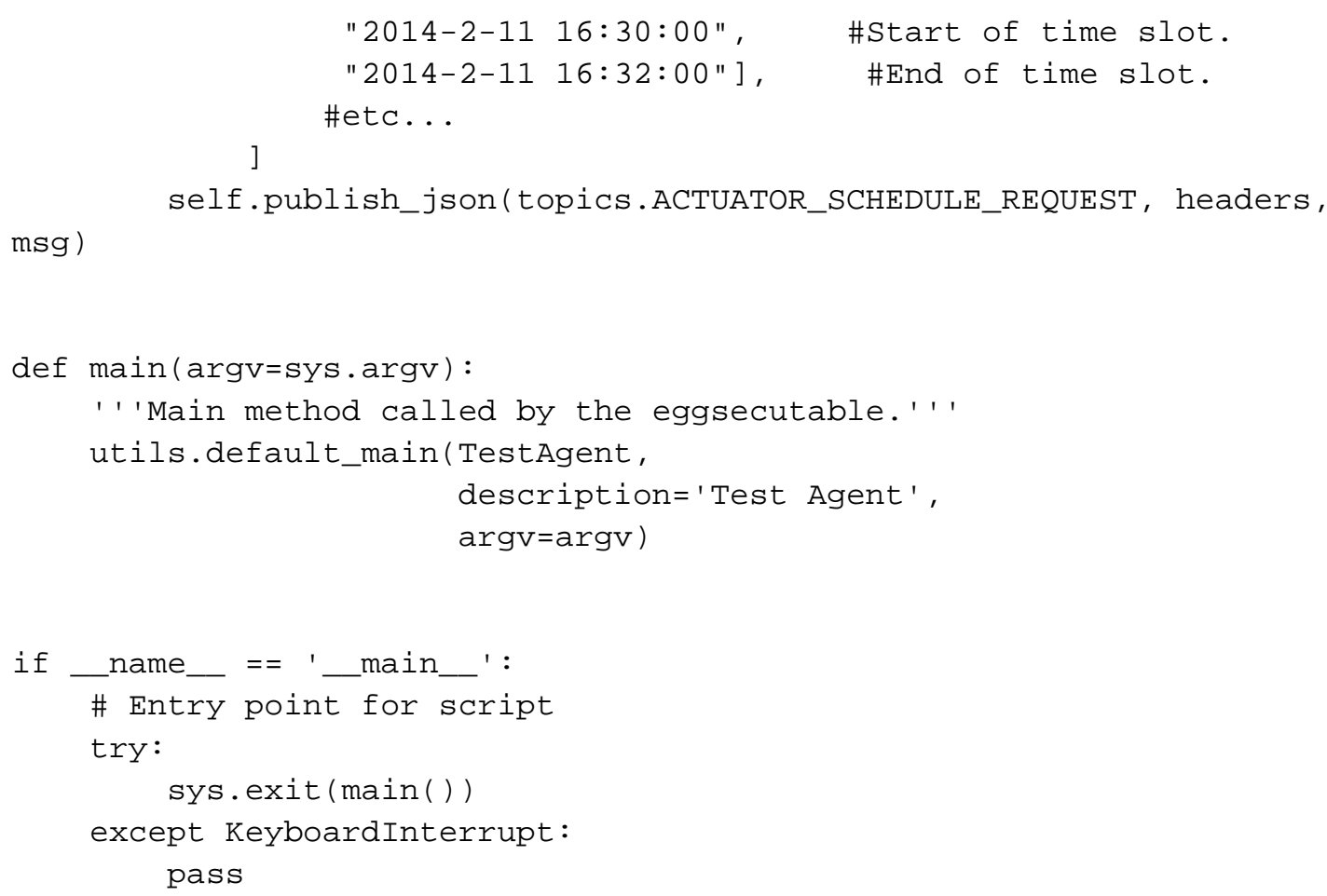


Appendix 
$\square$ VOLTTRON / volttron

\begin{tabular}{l|l|l|l|} 
Star & 0 & $\square$ Fork \\
\hline
\end{tabular}

Home Pages History

Home

Clone URL

\section{Welcome to the VOLTTRON(tm)}

\section{wiki.}

The project is in transition from another repository and wiki. We apologize for any

inconvenience during this phase.

As the wiki is being updated, you may run into problems with missing or confusing pages.

Transactional Network Platform Overview

Getting started with Transactional Network Development

\section{ExampleAgents}

Agent Development

Established Topics

Working with the Actuator Agent

DataOrganization

PlatformCommands 


\section{Note: As part of this change, the project directory has changed from rtunetwork to} volttron.

VOLTTRON is an open source agent platform designed to enable researchers to interact with devices and appliances without having to write drivers themselves

The main features of the VOLTTRON platform are:

- Open, flexible and modular software platform

- Ease of application development

- Interoperable across vendors and applications

- Isolates power and control system complexities from developers

- Object oriented, modern software development environment

- Language agnostic. Does not tie the applications to a specific language such as Java

- Broad device and control systems protocols support built-in

- ModBUS, BACNet, and others

- Multiple types of controllers and sensors

- Low CPU, memory and storage footprint requirements

- Supports non-Intel CPUs

\section{New to VOLTTRON?}

If you are new to VOLTTRON, please take a look at the VOLTTRON User's Guide for instructions on how to get started. This guide will walk you through getting the code, configuration, and running an example agent application.
PlatformStartup

PlatformServiceAgents

Working with the RTU Controller

Logging

ChangeLogs

Multi-building Messaging

BacnetDriver 


\section{Reference Material}

ATES 2012 Paper Initial paper. Presented at ASME 2011

AAMAS 2013 Demo

Grid and Vehicles paper

VOLTTRON Overview Video

\section{Transactional Network Documentation}

Transactional Network Platform Overview

Getting started with Transactional Network Development

ExampleAgents

Agent Development

Established Topics

Working with the Actuator Agent

DataOrganization

PlatformCommands

PlatformStartup

PlatformServiceAgents

Working with the RTU Controller 
Logging

ChangeLogs

Multi-building Messaging

BacnetDriver

Last edited by jhaack, April 23, 2014 


\section{Development Prerequisites}

\section{Required Sofware: Linux}

The following packages will need to be installed if they are not already:

- Install git: sudo apt-get install git

- Install Python DevTools: sudo apt-get install python-dev

- Install g++: sudo apt-get install g++

- Install libevent-dev: sudo apt-get install libevent-dev

If you have an agent which requires the pyodbc package, install the following:
Page History

Clone URL

Transactional Network Platform Overview

Getting started with Transactional Network Development

ExampleAgents

Agent Development

Established Topics

Working with the Actuator Agent

DataOrganization

PlatformCommands 
- Install FreeTDS: sudo apt-get install freetds-bin

- Install Unix ODBC: sudo apt-get install unixodbc-dev

\section{PlatformStartup}

PlatformServiceAgents

Working with the RTU Controller

Logging

ChangeLogs

Multi-building Messaging

BacnetDriver 


\section{Eclipse Dev Environment}

\section{VOLTTRON Development in Eclipse}

This page is being updated to reflect the change from Mercurial to Git

The Eclipse IDE has a Python plugin which is already installed in the VOLTTRON VM. In order to setup Eclipse outside the VM, download the IDE from: http://www.eclipse.org/ or through the Software Manager in Linux.

The VOLTTRON code is stored in a git repository. There is a plugin available for Eclipse that makes development more convenient (note: you must have Mercurial already installed on the
Page History

Clone URL

Transactional Network Platform Overview Getting started with Transactional Network Development

ExampleAgents

Agent Development

Established Topics

Working with the Actuator Agent

DataOrganization

PlatformCommands 
system and have built the project):

- Help -> Install New Software

- Click on the "Add" button

- For name use: Git

- For location: http://download.eclipse.org/egit/updates

- After hitting OK, check the box for: Check Select All

- Click through Next, Agree to Terms, then Finish

- Allow Eclipse to restart

After installing Eclipse, you must add the PyDev plugin to the environment. In Eclipse:

- Help -> Install New Software

- Click on the "Add" button

- For name use: PyDev

- For location: http://pydev.org/updates

- Check the box for PyDev

- Click through Next, Agree to Terms, Finish

- Allow Eclipse to restart

The project can now be checked out from the repository into Eclipse.

- Window -> Show View -> Other -> Mercurial -> Mercurial Repositories

- In the Mercurial Repositories View, click on the New Repositories button

- For Repository location, enter hg clone https://bitbucket.org/berkeleylab/rtunetwork

- Enter your username and password, hit ok

- Right-click on the repository, select Clone

- Enter username/password if not filled in

- Change clone directory name if desired (wiki assumes use of the default)

- Next, Finish

- The project must now be built outside Eclipse. Please follow the directions here and skip

\section{PlatformStartup}

PlatformServiceAgents

Working with the RTU Controller

Logging

ChangeLogs

Multi-building Messaging

BacnetDriver 
the hg clone portion.

- After changing the filesystem outside Eclipse, right-click on the project name and select Refresh

- PyDev must now be pointed at the correct version of Python.

- Window-> Preferences

- Expand PyDev

- Select Interpreter-Python

- Hit New

- Hit Browse and browse to the Python in the bin directory of the rtunetwork project.

Then hit Ok

- Select All, then Hit Ok

- You may need to redo this after a platform update and buildout

- In the Project/PackageExplorer view on the left, right-click on the project, PyDev-> Set as PyDev Project

- Switch to the PyDev perspective (if it has not already switched), Window -> Open

Perspective -> PyDev

Eclipse should now be configured to use the project's environment.

To test the installation:

- Setup a run configuration for the platform

- In the Package Explorer view, open the bin folder

- Righ-click on volttron-lite and select Run As -> Python Run (this will create a run configuration but fail)

- On the menu bar, pick Run -> Run Configurations...

- Under Python Run pick "rtunetwork volttron-lite"

- Click on the Argument tab

- Change Working Directory to Default

- In the Program arguments box, add: "-c dev-config.ini" This sets up running the 
platform in a development mode

- Click Run, this launches the platform. If the run does not succeed, click the all stop icon (two red boxes overlaid) on the console and then retry.

- Setup a run configuration for the ListenerAgent

- In the Package Explorer view, open Agents -> ListenerAgent --> listener

- Righ-click on listeneragent.py and select Run As -> Python Run (this will create a run configuration but fail)

- On the menu bar, pick Run -> Run Configurations...

- Under Python Run pick "rtunetwork listeneragent.py"

- Click on the Argument tab

- Change Working Directory to Default

- In the Program arguments box, add: "--config

Agents/ListenerAgent/listeneragent.launch.json --pub ipc:///tmp/volttron-lite-agent-

publish --sub ipc:///tmp/volttron-lite-agent-subscribe" This launches the agent with the dev settings the platform is using

- Click Run, this launches the agent

- You should see the agent start to publish and receive its own heartbeat message

Last edited by jhaack, April 23, 2014 


\section{VOLTTRON / volttron}

Home Pages History

\section{Building The Project}

\section{Building the Project}

The Volttron Lite project includes scripts which automatically pull down dependencies and build the libraries: bootstrap is a one-time use script. Use it after the initial checkout, then use "bin/buildout -N" after that.

Ensure you have installed the required packages before proceeding. Especially if you intend to develop in Eclipse, we recommend creating a directory: /workspace In this directory:

- git clone https://github.com/VOLTTRON/volttron
Page History

Clone URL

Transactional Network Platform Overview

Getting started with Transactional Network Development

ExampleAgents

Agent Development

Established Topics

Working with the Actuator Agent

DataOrganization

PlatformCommands 
cd volttron

- ./bootstrap

- If bootstrap fails before finishing (for instance from a timeout), run: bin/buildout -N

Note: If bootstrap or buildout fails, try "bin/buildout -N" again. Also, some packages (especially numpy) can be very verbose when they install. Please wait for the wall of text to finish.

To test that installation worked, start up the platform:

- Edit the dev-config.ini file to ensure the paths match up to your installation

- bin/volttron-lite -c dev-config.ini

- If it starts with no errors then your setup is correct

- If you are developing in Eclipse, then you should update the Python path at this point. See: EclipseDevEnvironment

To test agent deployment and messaging, build and deploy ListenerAgent: From the rtunetwork directory

- volttron/scripts/build-agent.sh ListenerAgent

- chmod +x Agents/listeneragent-0.1-py2.7.egg

- bin/volttron-ctrl install-executable Agents/listeneragent-0.1-py2.7.egg bin/

- bin/volttron-ctrl load-agent Agents/ListenerAgent/listeneragent.launch.json

- bin/volttron-ctrl start-agent listeneragent.launch.json

- To see that it's running: bin/volttron-ctrl list-agents

Last edited by jhaack, April 23, 2014 
$\square$ VOLTTRON / volttron

Home Pages History

\section{Agent Development}

\section{Agent Creation Walkthrough}

It is recommended that developers look at the ListenerAgent before developing their own agent. That agent expresses the basic functionality this example with walk through and being familiar with the concepts will be useful.

\section{Created Folders}

- In the Agents directory, create a new folder TestAgent

- In TestAgent, create a new folder tester, this is the package where our python code will be
Page History

Clone URL

Transactional Network Platform Overview Getting started with Transactional Network Development

\section{ExampleAgents}

Agent Development

Established Topics

Working with the Actuator Agent

DataOrganization

PlatformCommands 
created

\section{Create Agent Code}

- In tester, create a file called " $\langle$ u $>$ init $</ u\rangle$.py " which tells Python to treat this folder as a package

- In the tester package folder, create the file testagent.py

- Create a class called TestAgent

- Import the packages and classes we will need:

import sys

from volttron.lite.agent import BaseAgent, PublishMixin

from volttron.lite.agent import utils, matching

from volttron.lite.messaging import headers as headers mod

- - This agent will extend BaseAgent to get all the default functionality

- Since we want to publish we will use the PublishMixin. Mixins should be put first in the class definition

class TestAgent(PublishMixin, BaseAgent):

- We don't need to add anything to the BaseAgent init method so we will not create our own

\section{Setting up a Subscription}

We will set our agent up to listen to heartbeat messages (published by ListenerAgent). Using the matching package, we declare we want to match all topics which start with

"heartbeat/listeneragent". This will give us all heartbeat messages from all listeneragents but no others.
PlatformStartup

PlatformServiceAgents

Working with the RTU Controller

Logging

ChangeLogs

Multi-building Messaging

BacnetDriver 
@match_start ( 'heartbeat/listeneragent' )

def on_heartbeat_topic(self, topic, headers, message, match):

print "Testagent got \nTopic: \{topic\}, \{headers\}, Message:

\{message\}".format (topic=topic, headers=headers, message=message)

\section{Argument Parsing and Main}

Our agent will need to be able to parse arguments being passed on the command line by the agent launcher. Use the utils.default_main method to handle argument parsing and other default behavior. Create a main method which can be called by the launcher.

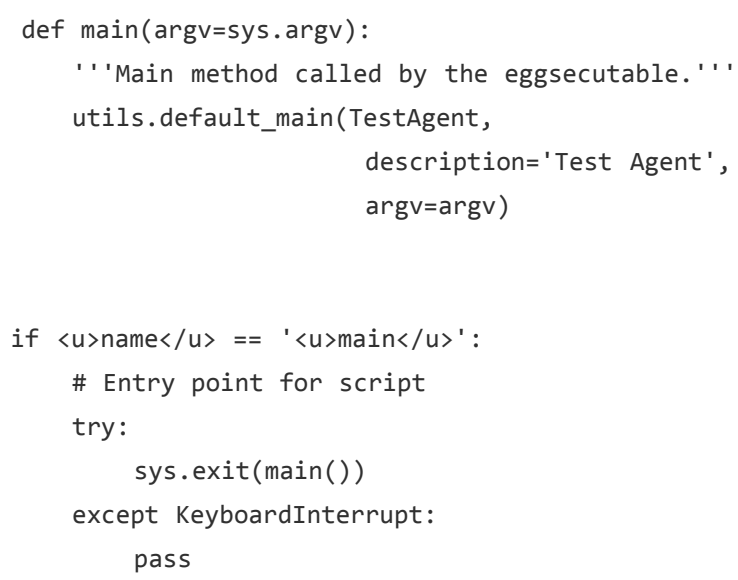

\section{Create Support Files for Agent}

Volttron-Lite agents need some configuration files for packaging, configuration, and launching.

\section{Packaging Configuration}


In the TestAgent folder, create a file called "setup.py" (or copy the setup.py in ListenerAgent) which we will use to create an eggsecutable. This file sets up the name, version, required packages, method to execute, etc. for the agent. The packaging process will also use this information to name the resulting file.

from setuptools import setup, find_packages

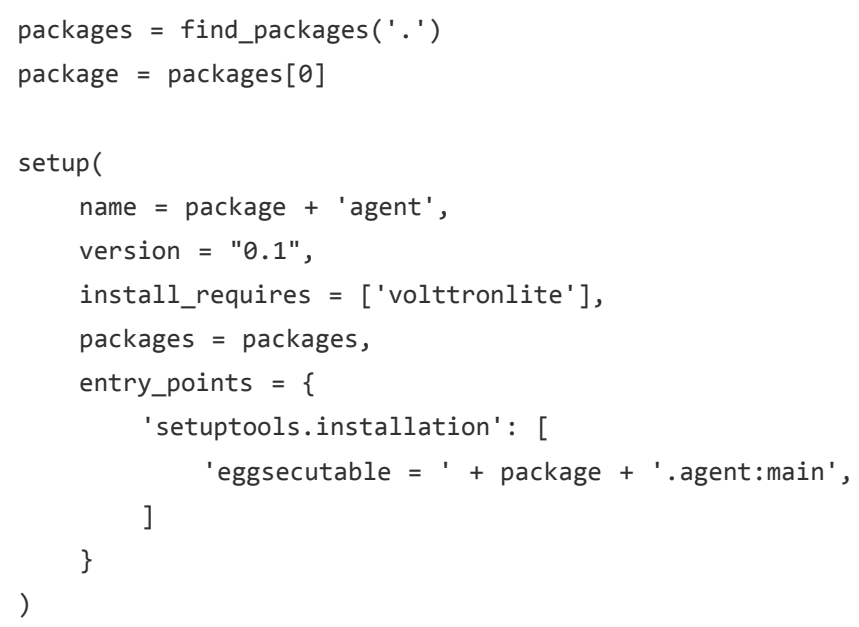

\section{Launch Configuration}

In TestAgent, create a file called "testagent.launch.json". This is the file the platform will use to launch the agent. It can also contain configuration information for the agent.

\section{For TestAgent}

\{

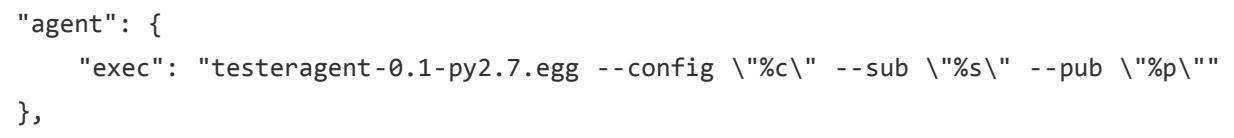


"agentid": "Test1",

"message": "hello"

\}

\section{Packaging Agent}

The agent code must now be packaged up for use by the platform. The build-agent.sh script will build the eggsecutable package using the setup.py file we defined earlier.

From the rtunetwork directory: "scripts/build-agent.sh TestAgent".

This creates an egg file in the Agents directory which, along with the launch configuration file, would be sent to a deployed platform for installation. For local testing, you may need to change permissions on the file: "chmod +x Agents/testeragent-.1-py2.7.egg"

\section{Testing the Agent}

\section{From the Command Line}

With the platform running, execute the following steps:

- Install the executable into the platform: "bin/volttron-ctrl install-executable Agents/testeragent-.1-py2.7.egg"

- Load the agent configuration file: "bin/volttron-ctrl load-agent Agents/TestAgent/testagent.launch.json"

- Start the platform by running "bin/volttron-lite -c dev-config.ini -I volttron.log -v -v"

- Launch the agent by running "bin/volttron-ctrl start_agent testagent.launch.json"

- Check that it is running: "bin/volttron-ctrl list-agents"

- Check the log file for messages: "tail volttron.log" 


\section{In Eclipse}

- If you are working in Eclipse, create a run configuration for TestAgent based on the ListenerAgent configuration in EclipseDevEnvironment.

- Launch the platform

- Launch the TestAgent

- Launch the ListenerAgent

TestAgent should start receiving the heartbeats from ListenerAgent

Last edited by Jereme Haack, April 05, 2014 


\section{VOLTTRON / volttron}

Home Pages History

\section{topics}

\section{Messaging}

Agents in VOLTTRON Lite communicate with each other using a publish/subscribe mechanism built on the Zero MQ Python library. This allows for great flexibility as topics can be created dynamically and the messages sent can be any format as long as the sender and receiver understand it. An agent with data to share publishes to a topic, then any agents interested in that data subscribe to that topic.

While this flexibility is powerful, it also could also lead to confusion if some standard is not followed. The current conventions for communicating in the VOLTTRON Lite are:
Page History

Clone URL

Transactional Network Platform Overview

Getting started with Transactional Network Development

ExampleAgents

Agent Development

Established Topics

Working with the Actuator Agent

DataOrganization

PlatformCommands 
- - Topics and subtopics follow the format: topic/subtopic/subtopic

- Subscribers can subscribe to any and all levels. Subscriptions to "topic" will include messages for the base topic and all subtopics. Subscriptions to "topic/subtopic1" will only receive messages for that subtopic and any children subtopics. Subscriptions to empty string ("'") will receive ALL messages. This is not recommended.

- All agents should subscribe to the "platform" topic. This is the topic the VOLTTRON Lite will use to send messages to agents, such as "shutdown".

Agents should set the "From" header. This will allow agents to filter on the "To" message sent back. This is especially useful for requests to the ArchiverAgent so agents do not receive replies not meant for their request.

\section{Topics}

\section{In VOLTTRON Lite}

- - platform - Base topic used by the platform to inform agents of platform events

- platform/shutdown - General shutdown command. All agents should exit upon receiving this. Message content will be a reason for the shutdown

- platform/shutdown_agent - This topic will provide a specific agent id. Agents should subscribe to this topic and exit if the id in the message matches their id.

\section{RTU Controller Agent Topics}

See the documentation for the ActuatorAgent.

Last edited by Jereme Haack, April 05, 2014 
$\square$ VOLTTRON / volttron

\begin{tabular}{l|l|l|l|} 
Star & 0 & $\square$ Fork & 1
\end{tabular}

Home Pages History

\section{ActuatorAgent}

\section{Actuator Agent}

This agent is used to access the control points of a controller. Agents may request scheduled times, called Tasks, to interact with one or more RTUs.

This is handled via pub/sub. The available points for a Catalyst 472 are detailed here.

See the ExampleControllerAgent for an example of using the ActuatorAgent.

\section{Actuator Agent Communication}

Page History

Clone URL

Transactional Network Platform Overview

Getting started with Transactional Network Development

ExampleAgents

Agent Development

Established Topics

Working with the Actuator Agent

DataOrganization

PlatformCommands 
Scheduling and canceling a Task.

Interacting with an RTU via the ActuatorAgent.

AcutatorAgent responses to a schedule or cancel request.

Schedule state announcements.

What happens when a running Task is preempted.

Setup heartbeat signal for a device.

ActuatorAgent configuration.

Notes on programming agents to work with the ActuatorAgent

Last edited by Jereme Haack, April 05, 2014
PlatformStartup

PlatformServiceAgents

Working with the RTU Controller

Logging

ChangeLogs

Multi-building Messaging

BacnetDriver 


\section{ActuatorScheduleRequest}

\section{Requesting Schedule Changes}

For information on responses see AcutatorAgent responses to a schedule or cancel requests.

The actuator agent expects all messages to be JSON and will parse them accordingly. Use publish_json to send messages where possible.

\section{Scheduling a Tas}

An agent can request a task schedule by publishing to the "RTU/actuators/schedule/request"
Page History

Clone URL

Transactional Network Platform Overview

Getting started with Transactional Network Development

ExampleAgents

Agent Development

Established Topics

Working with the Actuator Agent

DataOrganization

PlatformCommands 


\section{\#python}

\{

'type': 'NEW_SCHEDULE',

'requesterID': 〈Agent ID>, \#The name of the requesting agent.

taskID': <unique task ID>, \#The desired task ID for this task. It must be unique among all other scheduled tasks.

'priority': 〈task priority>, \#The desired task priority, must be 'HIGH', 'LOW', or 'LOW_PREEMPT'

\}

with the following message (before converting to json):

\section{\#python}

[

["campus/building/device1", \#First time slot.

"2013-12-06 16:00:00", \#Start of time slot.

"2013-12-06 16:20:00"], \#End of time slot.

["campus/building/device1", \#Second time slot.

"2013-12-06 18:00:00", \#Start of time slot.

"2013-12-06 18:20:00"], \#End of time slot.

["campus/building/device2", \#Third time slot.

"2013-12-06 16:00:00", \#Start of time slot.

"2013-12-06 16:20:00"], \#End of time slot.

\#etc...

]

\section{Points on Task Scheduling}

- Everything in the header is required.

- A Task schedule must have at least one time slot.

- The start and end times are parsed with dateutil's date/time parser. The default string

\section{PlatformStartup}

PlatformServiceAgents

Working with the RTU Controller

Logging

ChangeLogs

Multi-building Messaging

BacnetDriver 
representation of a python datetime object will parse without issue.

- Two Tasks are considered conflicted if at least one time slot on a device from one task overlaps the time slot of the other on the same device.

- The start or end (or both) of a requested time slot on a device may touch other time slots without overlapping and not be conflicted.

- A request must not conflict with itself.

- If something goes wrong see this failure string list for an explanation of the error.

\section{Task Priorities}

HIGH: :

This Task cannot be preempted under any circumstance. This task may preempt other conflicting preemptable Tasks. LOW: : This Task cannot be preempted once it has

started. A Task is considered started once the earliest time slot on any device has been rea ched. This Task may not preempt other Tasks. LOW_PREEMPT: :

This Task may be preempted at any time. If the Task is preempted once it has begun running an y current time slots will be given a grace period (configurable in the ActuatorAgent configurat ion file, defaults to 60 seconds) before being revoked. This Task may not preempt other Tas ks.

\section{Canceling a Task}

A task may be canceled by publishing to the "RTU/actuators/schedule/request" topic with the following header:

\#python

type': 'CANCEL_SCHEDULE',

'requesterID': 〈Agent ID>, \#The name of the requesting agent.

taskID': <unique task ID>, \#The desired task ID for this task. It must be unique among all other scheduled tasks. 


\section{Points on Task Canceling}

- The requesterID and taskID must match the original values from the original request header.

- After a Tasks time has passed there is no need to cancel it. Doing so will result in a "TASK_ID_DOES_NOT_EXIST" error.

- If something goes wrong see this failure string list for an explanation of the error. 


\section{ActuatorValueRequest}

\section{ActuatorAgent Interaction}

Once an Task has been scheduled and the time slot for one or more of the devices has started an agent may interact with the device using the get and set topics.

Both get and set are responded to the same way. See [\#ActuatorReply Actuator Reply] below.

\section{Getting values}

While the sMap driver for a device should always be setup to periodically broadcast the state of
Page History

Clone URL

Transactional Network Platform Overview

Getting started with Transactional Network Development

ExampleAgents

Agent Development

Established Topics

Working with the Actuator Agent

DataOrganization

PlatformCommands 
a device you may want an up to the moment value for an actuation point on a device.

To request a value publish a message to the following topic:

\#python

'RTU/actuators/get/<full device path>/<actuation point $\rangle$ '

With this header:

\section{\#python}

\{

'requesterID': 〈Agent ID>

\section{Setting Values}

Value are set in a similar manner:

To set a value publish a message to the following topic:

\#python

'RTU/actuators/set/<full device path>/<actuation point>'

With this header:

\#python

'requesterID': 〈Agent ID>

\section{PlatformStartup}

PlatformServiceAgents

Working with the RTU Controller

Logging

ChangeLogs

Multi-building Messaging

BacnetDriver 
And the message contents being the new value of the actuator.

The actuator agent expects all messages to be JSON and will parse them accordingly. Use publish_json to send messages where possible. This is significant for Boolean values especially.

\section{Actuator Reply}

\#ActuatorReply The ActuatorAgent will reply to both get and set' on the value topic for an actuator:

\section{\#python}

'RTU/actuators/value/<full device path>/<actuation point>'

With this header:

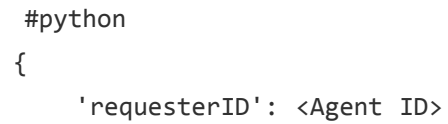

With the message containing the value encoded in JSON.

\section{Actuator Error Reply}

If something goes wrong the ActuatorAgent will reply to both get and set' on the error topic for an actuator:

\#python

'RTU/actuators/error/<full device path>/<actuation point>' 
With this header:

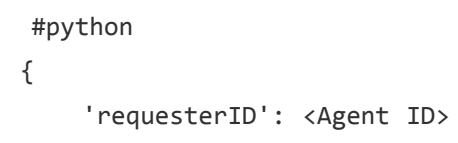

The message will be in the following form:

\#python

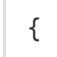

\section{Common Error Types}

LockError:: Returned when a request is made when we do not have permission to use a device. ( Forgot to schedule, preempted and we did not handle the preemption message correctly, ran out 0 $f$ time in time slot, etc...) ValueError: : Message missing or could not be parsed as JSON.

Other error types involve problem with communication between the ActuatorAgent and sMap. 
$\square$ VOLTTRON / volttron

\begin{tabular}{l|l|l|l|l|l} 
Star & 0 & Fork
\end{tabular}

Home Pages History

\section{ActuatorScheduleResponse}

\section{ActuatorAgent Respons}

In response to a Task schedule request the ActuatorAgent will respond on the topic

"RTU/actuators/schedule/response" with the header:

\section{\#python}

\{

'type' : 〈'NEW_SCHEDULE', 'CANCEL_SCHEDULE'>

'requesterID': 〈Agent ID from the request>,

'taskID': 〈Task ID from the request>

\}

Page History

Clone URL

Transactional Network Platform Overview

Getting started with Transactional Network Development

ExampleAgents

Agent Development

Established Topics

Working with the Actuator Agent

DataOrganization

PlatformCommands 


\begin{tabular}{|c|}
\hline And the message (after parsing the json): \\
\hline 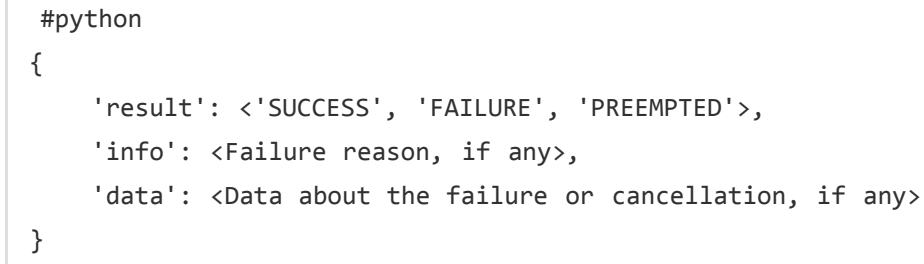 \\
\hline
\end{tabular}

\section{PlatformStartup}

PlatformServiceAgents

Working with the RTU Controller

Logging

ChangeLogs

Multi-building Messaging

BacnetDriver

The ActuatorAgent may publish cancellation notices for preempted Tasks using the "PREEMPTED" result.

\section{Preemption Data}

Preemption data takes the form:

\#python

\{

'agentID': <Agent ID of preempting task>,

'taskID': 〈Task ID of preempting task>

\section{Failure Reasons}

In many cases the ActuatorAgent will try to give good feedback as to why a request failed.

\section{General Failures}

INVALID_REQUEST_TYPE: : Request type was not "NEW_SCHEDULE" or "CANCEL_SCHEDULE". 
MISSING_TASK_ID:: Failed to supply a taskID. MISSING_AGENT_ID: : AgentID not supplied.

\section{Task Schedule Failures}

TASK_ID_ALREADY_EXISTS: : The supplied taskID already belongs to an existing task.

MISSING_PRIORITY:: Failed to supply a priority for a Task schedule request.

INVALID_PRIORITY:: Priority not one of "HIGH", "LOW", or "LOW_PREEMPT".

MALFORMED_REQUEST_EMPTY: : Request list is missing or empty.

REQUEST_CONFLICTS_WITH_SELF:: Requested time slots on the same device overlap.

MALFORMED_REQUEST: : Reported when the request parser raises an unhandled exception. The except ion name and info are appended to this info string.

CONFLICTS_WITH_EXISTING_SCHEDULES: : This schedule conflict with an existing schedules that it cannot preempt. The data item for the results will contain info about the conflicts in this fo rm (after parsing json):

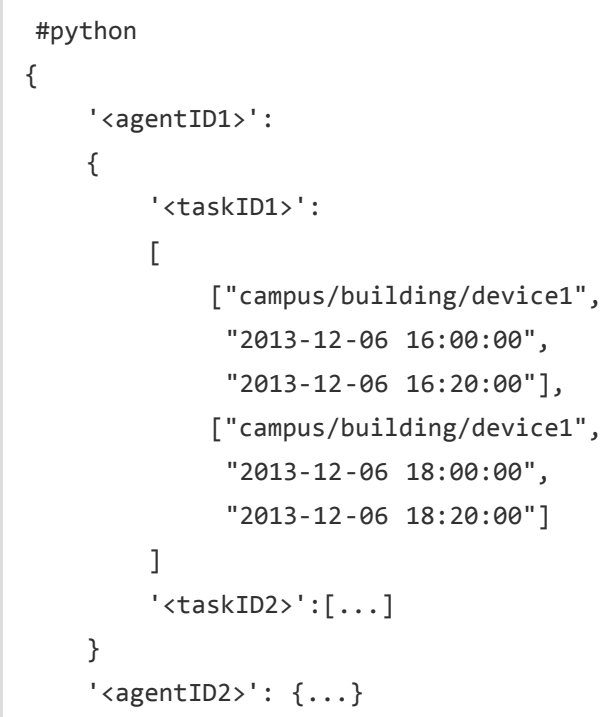




\section{Task Cancel Failures}

TASK_ID_DOES_NOT_EXIST: : Trying to cancel a Task which does not exist. This error can also occ ur when trying to cancel a finished Task.

AGENT_ID_TASK_ID_MISMATCH:: A different agent ID is being used when trying to cancel a Task.

Last edited by jhaack, April 22, 2014 


\section{ActuatorScheduleState}

\section{Schedule State Broadcast}

Periodically the ActuatorAgent will publish the state of all currently used devices.

For each device the ActuatorAgent will publish to an associated topic:

\section{\#python}

'RTU/actuators/schedule/announce/<full device path〉'

With the following header:
Page History

Clone URL

Transactional Network Platform Overview

Getting started with Transactional Network Development

ExampleAgents

Agent Development

Established Topics

Working with the Actuator Agent

DataOrganization

PlatformCommands 


\section{\#python}

'requesterID': <Agent with access>,

'taskID': 〈Task associated with the time slot>

'window': 〈Seconds remaining in the time slot>

\}

The frequency of the updates is configurable with the "schedule_publish_interval" setting.

\section{PlatformStartup}

PlatformServiceAgents

Working with the RTU Controller

Logging

ChangeLogs

Multi-building Messaging

BacnetDriver 
Home Pages History

\section{ActuatorSchedulePreemption}

\section{Task Preemption}

Both LOW and LOW_PREEMPT priority Tasks can be preempted. LOW priority Tasks may be preempted by a conflicting HIGH priority Task before it starts. LOW_PREEMPT priority Tasks can be preempted by HIGH priority Tasks even after they start.

When a Task is preempted the ActuatorAgent will publish to

"RTU/actuators/schedule/response" with the following header:
Page History

Clone URL

Transactional Network Platform Overview

Getting started with Transactional Network Development

ExampleAgents

Agent Development

Established Topics

Working with the Actuator Agent

DataOrganization

PlatformCommands 


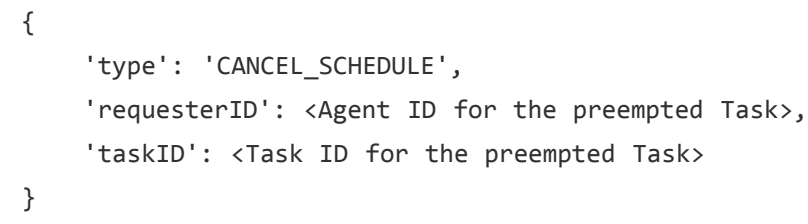

And the message (after parsing the json):

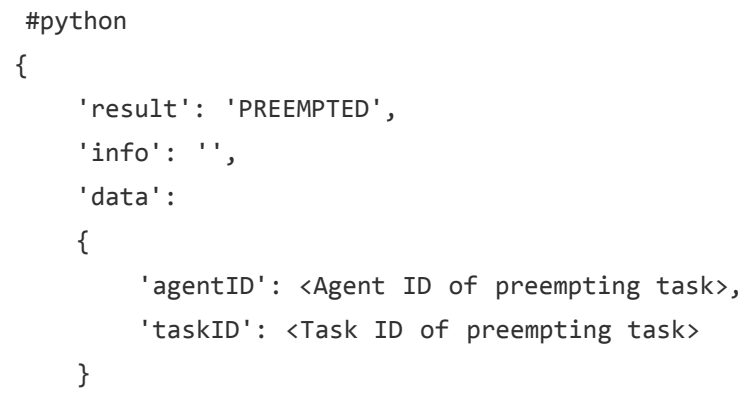

\section{Preemption Grace Time}

If a LOW_PREEMPT priority Task is preempted while it is running the Task will be given a grace period to clean up before ending. For every device which has a current time slot the window of remaining time will be reduced to the grace time. At the end of the grace time the Task will finish. If the Task has no currently open time slots on any devices it will end immediately.

\section{PlatformStartup}

PlatformServiceAgents

Working with the RTU Controller

Logging

ChangeLogs

Multi-building Messaging

BacnetDriver 


\section{VOLTTRON / volttron}

Home Pages History

\section{ActuatorHeartbeat}

Page History

Clone URL

\section{Heartbeat Signal}

The ActuatorAgent can be configured to send a heartbeat message to the device to indicate the platform is running. Ideally, if the heartbeat signal is not sent the device should take over and resume normal operation.

The configuration has two parts, the interval (in seconds) for sending the heartbeat and the specific point that should be modified each iteration.

The heart beat interval is specified with a global "heartbeat_interval" setting. The ActuatorAgent

Transactional Network Platform Overview
Getting started with Transactional Network
Development
ExampleAgents
Agent Development
Established Topics
Working with the Actuator Agent
DataOrganization
PlatformCommands


will automatically set the heartbeat point to alternating " 1 " and "0" values. Changes to the heartbeat point will be published like any other value change on a device.

\section{The heartbeat points are specified under "points" section of the configuration like so:}

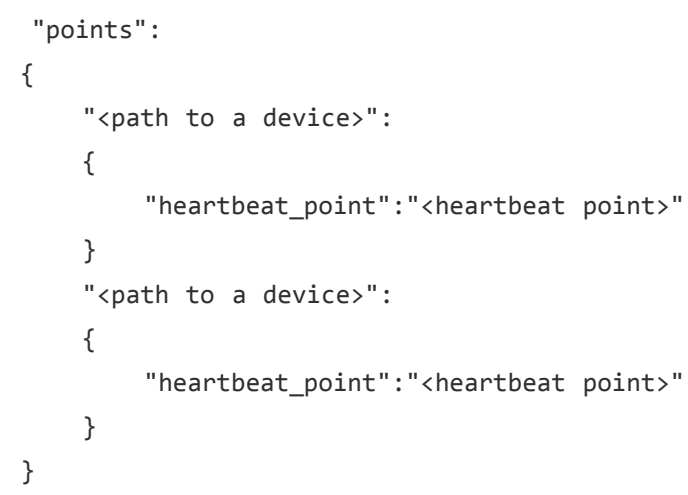

Note: this requires that the device has been setup with a heartbeat support. For Catalyst controllers check with TWT if you're not sure.

\section{PlatformStartup}

PlatformServiceAgents

Working with the RTU Controller

Logging

ChangeLogs

Multi-building Messaging

BacnetDriver 


\section{ActuatorConfig}

\section{ActuatorAgent Configuration}

url:: Url which will be used to communicate with the sMap drivers running in twistd.

schedule_publish_interval:: Interval between published schedule

announcements in seconds. Defaults to 30 . heartbeat_interval:: Interval between

heartbeats sent to a device in seconds. Defaults to 30 .

preempt_grace_time: : Minimum time given to Tasks which have been preempted to clean up in seco nds. Defaults to 60 .

schedule_state_file:: File used to save and restore Task states if the ActuatorAgent restarts for any reason. File will be created if it does not exist when it is needed.
Page History

Clone URL

Transactional Network Platform Overview

Getting started with Transactional Network Development

ExampleAgents

Agent Development

Established Topics

Working with the Actuator Agent

DataOrganization

PlatformCommands 
points: Devices and points which are specified as heartbeat points .

\section{Sample configuration file}

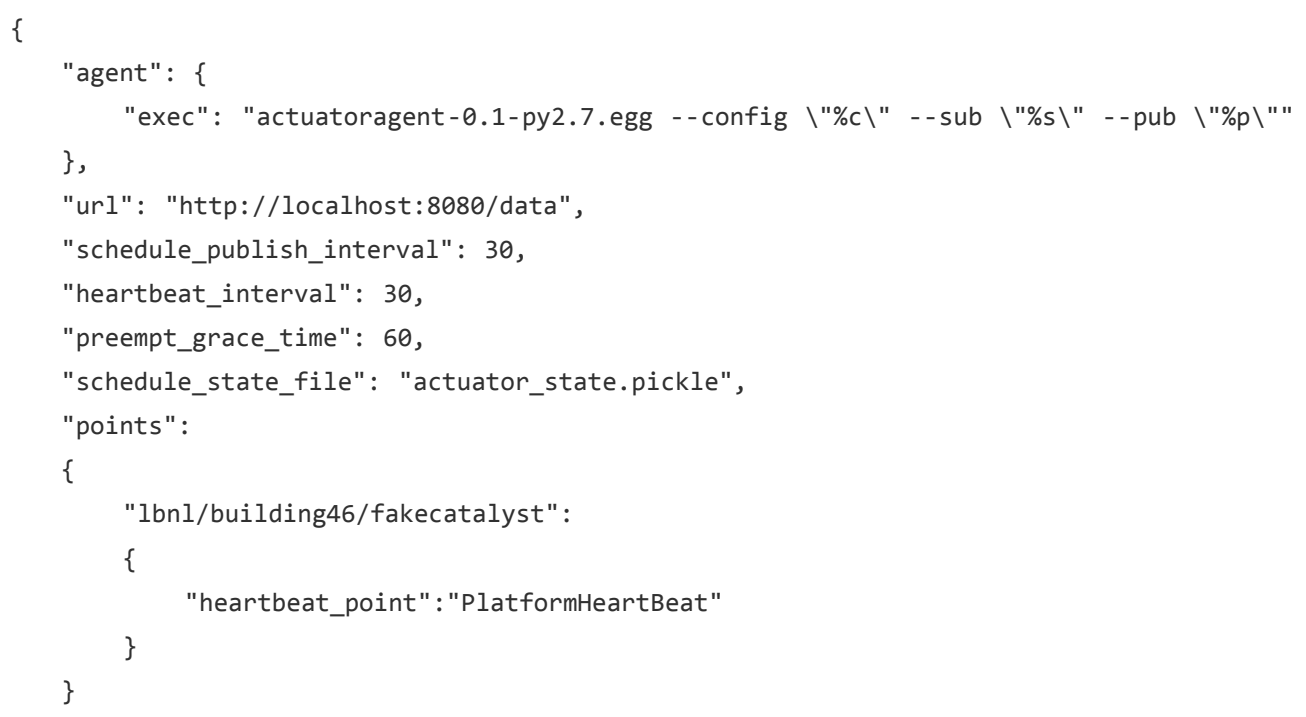

PlatformStartup

PlatformServiceAgents

Working with the RTU Controller

Logging

ChangeLogs

Multi-building Messaging

BacnetDriver 


\section{Actuator Agent Programming Notes}

\section{Notes on Working With the ActuatorAgent}

- An agent can watch the window value from device state updates to perform scheduled actions within a timeslot.

- If an Agent's Task is LOW_PREEMPT priority it can watch for device state updates where the window is less than or equal to the grace period (default 60.0).

- When considering if to schedule long or multiple short time slots on a single device:

- Do we need to ensure the device state for the duration between slots?

- Yes. Schedule one long time slot instead.

- No. Is it all part of the same Task or can we break it up in case there is a conflict with
Page History

Clone URL

Transactional Network Platform Overview Getting started with Transactional Network Development

ExampleAgents

Agent Development

Established Topics

Working with the Actuator Agent

DataOrganization

PlatformCommands 
one of our time slots?

- When considering time slots on multiple devices for a single Task:

- Is the Task really dependent on all devices or is it actually multiple Tasks?

- When considering priority:

- Does the Task have to happen on an exact day?

- No. Consider LOW and reschedule if preempted.

- Yes. Use HIGH.

- Is it problematic to prematurely stop a Task once started?

- No. Consider LOW_PREEMPT and watch the device state updates for a small window value.

- Yes. Consider LOW or HIGH.

- If an agent is only observing but needs to assure that no another Task is going on while taking readings it can schedule the time to prevent other agents from messing with a devices state. The schedule updates can be used as a reminder as to when to start watching.

- Any device, existing or not, can be scheduled. This allows for agents to schedule fake devices to create reminders to start working later rather then setting up their own internal timers and schedules.

\section{PlatformStartup}

PlatformServiceAgents

Working with the RTU Controller

Logging

ChangeLogs

Multi-building Messaging

BacnetDriver 
Home Pages History

\section{Platform Commands}

\section{Platform Command}

To startup the platform, specify the config file with -c. To specify a log file, use: -I

bin/volttron-lite -c config.ini -l volttron.log

Full options:

Volttron Lite agent platform daemon

optional arguments:

Page History

Clone URL

Transactional Network Platform Overview

Getting started with Transactional Network Development

ExampleAgents

Agent Development

Established Topics

Working with the Actuator Agent

DataOrganization

PlatformCommands 


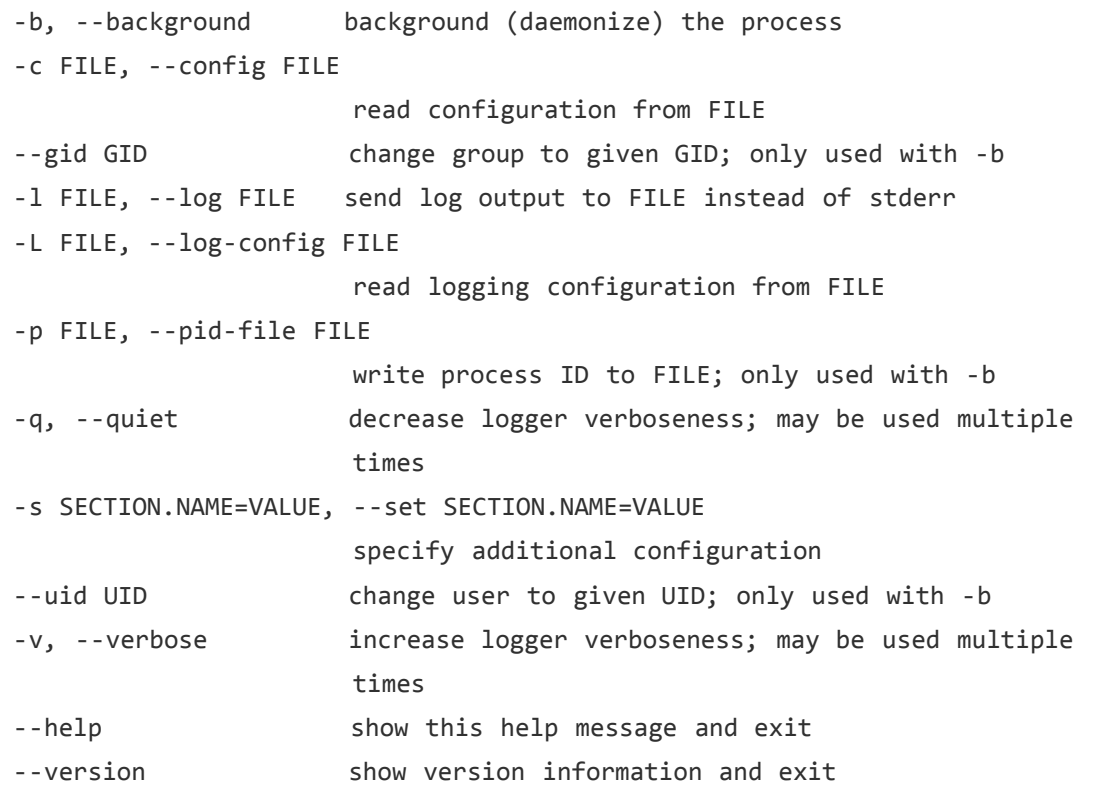

The platform can accept commands during operation using bin/volttron-ctrl

usage: volttron-ctrl command [options]

Control volttron and perform other related tasks

list of commands:

disable-agent

enable-agent

prevent agent from starting automatically

help enable agent to start automatically

install-executable install agent executable

list-agents list agents

list-executables

list agent executables

load-agent install agent launch file

remove-executable run-agent remove agent executable run agent(s) defined in config file(s) 


$\begin{array}{ll}\text { shutdown } & \text { stop all agents } \\ \text { start-agent } & \text { start installed agent } \\ \text { stop-agent } & \text { stop running agent } \\ \text { unload-agent } & \text { remove agent launch file }\end{array}$

Use $\langle\mathrm{tt}\rangle$ volttron-ctrl $-\mathrm{v}</ \mathrm{tt}\rangle$ to show aliases and global options.

Last edited by Jereme Haack, April 05, 2014 


\section{VOLTTRON / volttron}

Home Pages History

\section{ExampleSetup}

- Modify the driver.ini file

- Add your SMAP Key to the url

- Name your collection source

- Give your collection a uuid

- Enter your collection paths and metadata

- If you are deploying the WeatherAgent update settings.py with your Weather Underground key

- By default the Catalyst registers are setup to work with a 372 with the latest changes from TWT, if you have an older unit use the catalystreg.csv.371 file

Start the smap driver

Page History

Clone URL

Transactional Network Platform Overview

Getting started with Transactional Network Development

ExampleAgents

Agent Development

Established Topics

Working with the Actuator Agent

DataOrganization

PlatformCommands 
. bin/activate

twistd - $n$ smap driver.ini

With the platform already running:

- Build agent eggs

- Make egg executable

- Install egg into bin (must not already exist there)

- Load agent config file

- Enable agent for autostart

volttron/scripts/build-agent.sh ArchiverAgent

chmod +x Agents/archiveragent-0.1-py2.7.egg

bin/volttron-ctrl install-executable Agents/archiveragent-0.1-py2.7.egg

bin/volttron-ctrl load-agent Agents/ArchiverAgent/archiver-deploy.service

bin/volttron-ctrl enable-agent archiver-deploy.service

bin/volttron-ctrl list-agents

AGENT AUTOSTART STATUS

archiver-deploy.service enabled

volttron/scripts/build-agent.sh ActuatorAgent

chmod +x Agents/actuatoragent-0.1-py2.7.egg

bin/volttron-ctrl install-executable Agents/actuatoragent-0.1-py2.7.egg

bin/volttron-ctrl load-agent Agents/ActuatorAgent/actuator-deploy.service

bin/volttron-ctrl enable-agent actuator-deploy.service

Do the same things for WeatherAgent if you plan to deploy it.

Control Application Example Install one executable but multiple launch configuration files. Each instance of this agent will work with a different RTU.

\section{PlatformStartup}

PlatformServiceAgents

Working with the RTU Controller

Logging

ChangeLogs

Multi-building Messaging

BacnetDriver 
volttron/scripts/build-agent.sh SMDSAgent

chmod $+x$ Agents/SMDSagent-0.1-py2.7.egg

bin/volttron-ctrl install-executable Agents/SMDSagent-0.1-py2.7.egg

bin/volttron-ctrl load-agent Agents/SMDSAgent/smds-1bnl1.agent

bin/volttron-ctrl load-agent Agents/SMDSAgent/smds-lbn12.agent bin/volttron-ctrl load-agent Agents/SMDSAgent/smds-1bnl3.agent bin/volttron-ctrl load-agent Agents/SMDSAgent/smds-lbn14.agent bin/volttron-ctrl load-agent Agents/SMDSAgent/smds-1bn15.agent bin/volttron-ctrl load-agent Agents/SMDSAgent/smds-lbnl6.agent bin/volttron-ctrl load-agent Agents/SMDSAgent/smds-lbnl7.agent bin/volttron-ctrl load-agent Agents/SMDSAgent/smds-twt1.agent bin/volttron-ctrl load-agent Agents/SMDSAgent/smds-twt2.agent bin/volttron-ctrl load-agent Agents/SMDSAgent/smds-twt3.agent bin/volttron-ctrl load-agent Agents/SMDSAgent/smds-twt4.agent

bin/volttron-ctrl enable-agent smds-lbnl1.agent bin/volttron-ctrl enable-agent smds-lbnl2.agent bin/volttron-ctrl enable-agent smds-lbnl3.agent bin/volttron-ctrl enable-agent smds-lbnl4.agent bin/volttron-ctrl enable-agent smds-lbn15.agent bin/volttron-ctrl enable-agent smds-lbnl6.agent bin/volttron-ctrl enable-agent smds-lbnl7.agent bin/volttron-ctrl enable-agent smds-twt1.agent bin/volttron-ctrl enable-agent smds-twt2.agent bin/volttron-ctrl enable-agent smds-twt3.agent bin/volttron-ctrl enable-agent smds-twt4.agent

Restart platform and agents you have enable for autostart should start up.

Last edited by jhaack, April 22, 2014 


\section{VOLTTRON / volttron}

Home Pages History

\section{ArchiverAgent}

Page History

Clone URL

\section{Archiver Agent}

The SMDSAgent illustrates working with the ArchiverAgent.

publish('archiver/request/campus1/building1/realcatalyst1/CoolCall1',\{\},'(now -1h, now)')

publish('archiver/request/campus1/building1/realcatalyst1/CoolCall1',\{\},'(1374192541000.0,

1374193541000.0)')

The agent listens for messages on "archiver/request". The message that comes after is extracted and used in the Archiver query and represents the path to the value desired. There is 
also a source name that needs to be specified. This is currently part of the launch config. The data is returned as a list of lists:

1371851254000.0,1.0],[1371851314000.0,1.0],[1371851374000.0,1.0],[1371851434000.0,1.0],[ 1371851494000.0,1.0],[1371851554000.0,1.0],[1371851614000.0,1.0],[1371851674000.0,1.0],[ 1371851734000.0,1.0],[1371851794000.0,1.0],[1371851854000.0,1.0],[1371851914000.0,1.0],[ 1371851974000.0,1.0],[1371852034000.0,1.0],[1371852094000.0,1.0],[1371852154000.0,1.0],[ 1371852214000.0,1.0],[1371852274000.0,1.0],[1371852334000.0,1.0],[1371852394000.0,1.0],[ 1371852454000.0,1.0],[1371852514000.0,1.0],[1371852574000.0,1.0],[1371852634000.0,1.0],[ 1371852694000.0,1.0],[1371852754000.0,1.0],[1371852814000.0,1.0],[1371852874000.0,1.0],[ 1371852934000.0,1.0],[1371852994000.0,1.0],[1371853054000.0,1.0],[1371853114000.0,1.0],[ 1371853174000.0,1.0],[1371853234000.0,1.0],[1371853294000.0,1.0],[1371853354000.0,1.0],[ 1371853414000.0,1.0],[1371853474000.0,1.0],[1371853534000.0,1.0],[1371853594000.0,1.0],[ 1371853654000.0,1.0],[1371853714000.0,1.0],[1371853774000.0,1.0],[1371853834000.0,1.0],[ $1371853894000.0,1.0],[1371853954000.0,1.0],[1371854005000.0,1.0$

Currently, the format for the time is (start time, end time) as a string. In the example above, that requests data for the last hour. For each item in the list, the first element is the time stamp of the observation, and the second is the value. The smap archiver page indicates that you can use a variety of ways to specify the time, but l've only used unix time, and the now $-1 \mathrm{~h}$ constructs. This is what the smap page says:

You can select the time region queried using a range query, or a query relative to a reference time stamp. In all these cases, the reference times must either be a timestamp in units of UNIX milliseconds. The reference may be modified by appending a relative time string, using unix "at"-style specifications. You can for instance say now +1 hour or now $-1 \mathrm{~h}-5 \mathrm{~m}$ for the last 1:05. Available relative time quantities are days, hours, minutes, and seconds.

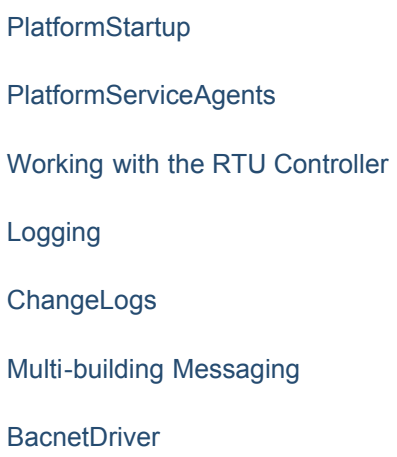




\section{VOLTTRON / volttron}

Home Pages History

\section{LoggerAgent}

The Logger agent will listen on the /logger topic for messages from other agents. These messages will then be pushed to the sMap historian. Data sources can be dynamically created by agents.

Page History

Clone URL

Transactional Network Platform Overview

Getting started with Transactional Network Development

ExampleAgents

Agent Development

Established Topics

Working with the Actuator Agent

PlatformCommands

PlatformStartup 
PlatformServiceAgents

Working with the RTU Controller

Logging

ChangeLogs

Multi-building Messaging

BacnetDriver 


\section{VOLTTRON / volttron}

\begin{tabular}{l|l|l|l|} 
Star & 0 & $\square$ Fork & 1
\end{tabular}

Home Pages History

\section{ControllerAccess}

\section{Controller Access}

There are configuration files you must edit to use keys generated for your lab. Do not check these files into the repository. You should keep them private.

The example smap driver ini file is "driver.ini" at the base of the project directory. Please edit this file to reflect your setup:

- ReportDeliveryLocation: requires an admin key from sMAP

- Metadata/SourceName: The name of the source for your catalyst

Page History

Clone URL

Transactional Network Platform Overview

Getting started with Transactional Network Development

ExampleAgents

Agent Development

Established Topics

Working with the Actuator Agent

PlatformCommands

PlatformStartup 
uuid: A unique identifier (can be any generated by any means)

When configuring a Modbus device be sure to setup the following:

- ip_address: IP address of the Modbus device.

- register_config: the csv file use to configure the Modbus device. You should specify the absolute path name of the configuration file. See [ModbusDriver Configure Modbus sMAP driver]

When configuring a BACnet device be sure to setup the following:

- target_address: Address of the BACnet device.

- self_address: IP address of the network interface used to communicate with the device.

This is required as the BACnet driver creates a virtual device to do it's communication and it communicates via UDP.

- register_config: the csv file use to configure the Modbus device. You should specify the absolute path name of the configuration file. See [BacnetDriver Configure BACnet sMAP driver]

[report $\theta]$

\#Insert your SMAP key after add

ReportDeliveryLocation = http://smap.lbl.gov/backend/add/〈INSERT YOUR KEY HERE $\rangle$

[/]

type $=$ Collection

Metadata/SourceName $=\langle$ PUT YOUR NAME HERE $\rangle$ Catalyst Data 2

uuid $=\langle$ PUT YOUR UUID HERE $\rangle$

[/campus1]

type $=$ Collection

Metadata/Location/Campus $=$ Campus Number 1

[/campus1/building1]

type $=$ Collection

\section{PlatformServiceAgents}

Working with the RTU Controller

Logging

ChangeLogs

Multi-building Messaging

BacnetDrive 


\section{[/campus1/building1/modbus1]}

type = volttron.drivers.modbus.Modbus

ip_address $=\langle$ PUT YOUR CATALYST IP HERE $\rangle$

Metadata/Instrument/Manufacturer $=\langle$ PUT INSTRUMENT MANUFACTURER HERE $\rangle$

Metadata/Instrument/ModelName $=\langle$ PUT INSTRUMENT MODEL HERE $\rangle$

\#port $=\langle$ DEVICE PORT if needed, defaults to 502 $\rangle$

\#slave_id = 〈SLAVE ID of modbus device if needed, defaults to $\theta\rangle$

\#see volttron/drivers/example.csv

\#for an example of a catalyst register config file

register_config $=\langle$ PUT YOUR REGISTER CONFIG HERE〉

[/campus1/building1/bacnet1]

type $=$ volttron.drivers.bacnet. BACnet

target_address $=\langle$ PUT YOUR BACNET DEVICE ADDRESS HERE $\rangle$

self_address $=\langle$ PUT IP OF INTERFACE USED TO COMMUNICATE WITH DEVICE (THIS COMPUTER IP) $\rangle$

Metadata/Instrument/Manufacturer $=\langle$ PUT INSTRUMENT MANUFACTURER HERE $\rangle$

Metadata/Instrument/ModelName $=\langle$ PUT INSTRUMENT MODEL HERE $\rangle$

\#see volttron/drivers/bacnet_example_config.csv

\#for an example of a bacnet register config file

register config $=\langle$ PUT YOUR REGISTER CONFIG HERE

[/campus1/building1/logger]

\#Currently this will only write to the file specified.

\#Hopefully logging to historian is forthcoming.

type = volttron.drivers.smap_logging.Logger

file $=$ 'test. $\log$

Once the ini file is configured:

- In a terminal from the rtunetwork directory

- Activate the project python

- . bin/activate (note the space)

- The sMAP driver relies on Twistd. Start this now: 


\section{- twistd -n smap}

Data should now be getting scraped from the Catalyst controller, published to the platform, and stored in the sMap historian. If you launch the ListenerAgent, you should see RTU data being published once a minute.

In order to set points, you must start the ActuatorAgent. Launch this as you would launch any other agent. In Eclipse, setup a run configuration with the following Program Arguments: $\{\{-$ config Agents/ActuatorAgent/actuatoragent.launch.json --pub ipc:///tmp/volttron-lite-agentpublish --sub ipc:///tmp/volttron-lite-agent-subscribe

\section{Please see the ExampleControllerAgent for making use of the ActuatorAgent.}

Last edited by kmonson, April 22, 2014 


\section{VOLTTRON / volttron}

Home Pages History

\section{Logging}

\section{Data Logging}

A mechanism for storing data in SMAP has been provided. The data logger is written as an SMAP driver, but receives information in a zero $M Q$ message sent to a topic prefixed with datalogger/log. The source name is configured at the time the logger starts up and is defined in the driver.ini file. The rest of the topic is extracted and used as the path in SMAP for the data point. If the path already exists, the time series items will be added to the bottom of the list. For example, if our source name is 'test data', and the topic we publish data to is datalogger/log/campus1/building1/testdata, then our data will be posted to time series under campus1/building1/testdata in the source name 'test data'.

Page History

Clone URL

Transactional Network Platform Overview Getting started with Transactional Network Development

ExampleAgents

Agent Development

Established Topics

Working with the Actuator Agent

PlatformCommands

PlatformStartup 
The Logger should be added to the SMAP configuration file alongside the Catalyst configuration. The location listed in the configuration file will not be used unless no path is given after datalogger/log.

\section{[/datalogger]}

type $=$ volttron.drivers.data_logger.DataLogger

interval $=1$

\section{Data Logging Format}

Data sent to the data logger should be sent as a JSON object that consists of a dictionary of dictionaries. The keys of the outer dictionary are used as the points in SMAP to store the data items. The inner dictionary consists of 2 required fields and 1 optional. The required fields are "Readings" and "Units". Readings contains the data that will be written to SMAP. It may contain either a single value, or a list of lists which consists of timestamp/value pairs. Units is a string that identifies the meaning of the scale values of the data. The optional entry is data_type, which indicates the type of the data to be stored. This may be either long or double.

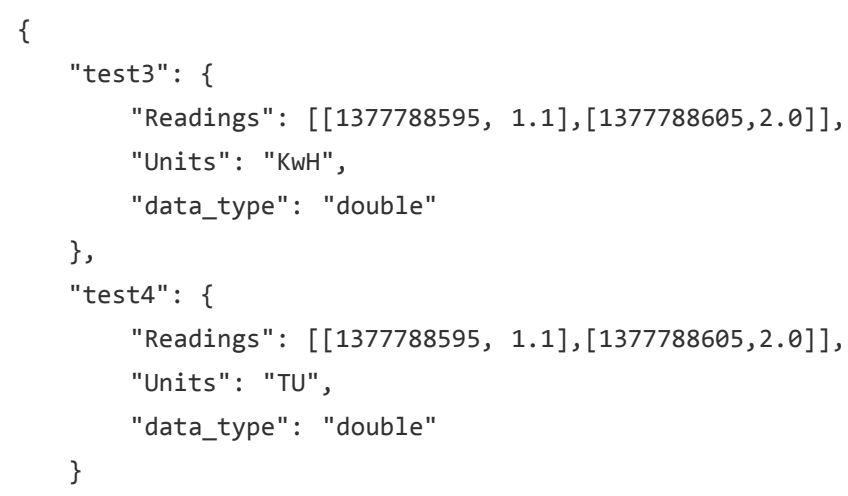




\section{Data Logger Response}

The data logger reports the status of the storage request to topic called datalogger/status. The response will either be success, or error, and the message will contain information on what failed if there was an error. In addition, the data logger looks in the message headers for a field called "from" to determine who should be the recipient of this message. The status message contains a header called "to" that uses the value retrieved from "from" so that agents may filter messages sent to datalogger/status using the match_headers decorator in a base agent derived agent.

\section{Example Code}

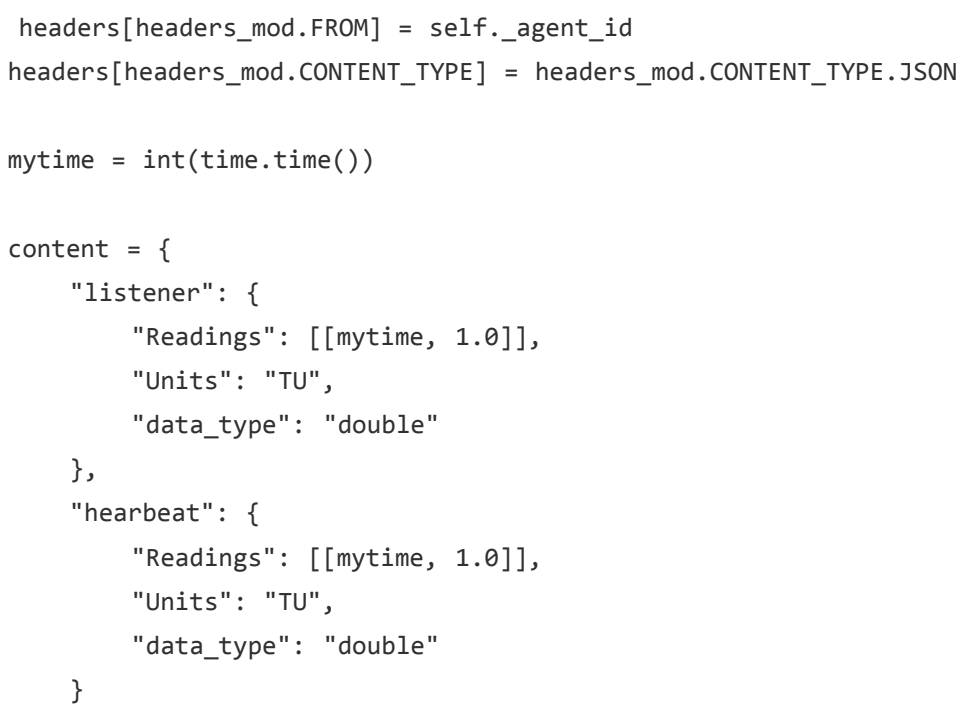


self.publish('datalogger/log/', headers, json.dumps(content)) 


\section{Multi-Building Messaging}

\section{Mult-building Messaging}

Multi-building messaging is implemented as a service-style agent. Its use is optional and can be enabled/disabled by simply enabling/disabling the multibuilding service agent. It is easily configured using the service launch file and provides several new topics for use in the local agent exchange bus.

\section{Configuration}

The agent launch file may contain the declarations below:
Page History

Clone URL

\section{Home}

Transactional Network Platform Overview

Getting started with Transactional Network Development

ExampleAgents

Agent Development

Established Topics

Working with the Actuator Agent

PlatformCommands 
building-publish-address: :

A ФMQ address used to publish to the building's message bus. Defaults to 'tcp://0.0.0.0:916 $1^{\prime}$.

building-subscribe-address: :

A $\emptyset \mathrm{MQ}$ address used to subscribe to the building's message bus. Defaults to 'tcp://0.0.0.0:9 $160^{\prime}$.

cleanup-period: :

Frequency, in seconds, to check for and close stale connections. Defaults to 600 seconds (1 0 minutes).

\section{hosts: :}

A mapping (dictionary) of building names to publish/subscribe addresses. Each entry is of $t$ he form:

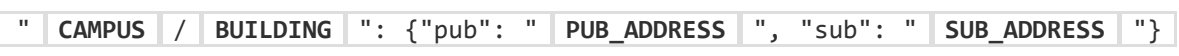

where PUB_ADDRESS and SUB_ADDRESS are ФMQ addresses.

uuid: :

A UUID to use in the Cookie header. If not given, one will be automatically generated.

\section{Sending and Receiving Inter-building Messages}

Three topics are provided for inter-building messaging:

building/recv/ CAMPUS / BUILDING /

Agents can subscribe to to this topic to receive messages sent to TOPIC at the building specified by CAMPUS / BUILDING .

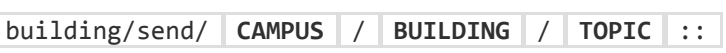

\section{PlatformStartup}

PlatformServiceAgents

Working with the RTU Controller

Logging

Multi-building Messaging

BacnetDriver 
Agents can send messages to this topic to have them forwarded to TOPIC at the building $s$ pecified by

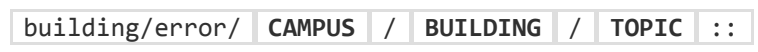

Errors encountered during sending/receiving to/from the above two topics will be sent over $t$ his topic.

\section{Limitations and Future Work}

Requires opening multiple listening ports:: It would be nice to multiplex all inter-

building communications over a single port to decrease the attack footprint and ease firewall a dministration.

There is no authentication, authorization, or encryption: :

Data is currently open to sniffing, modification, and all sorts of malicious activity.

Remote host lookup is kept in a static file:

Ideally, host lookup would be done through some central directory service, but that is not currently implemented. 


\title{
VOLTTRON / volttron
}

\begin{tabular}{l|l|l|l|} 
Star & 0 & $\square$ Fork & 1
\end{tabular}

\author{
Home Pages History
}

\section{Bacnet Driver}

Table of Contents

- Configuring BACnet sMAP driver

- PNNL Point Name

- Units

- BACnet Object Type

- Property

- Writable

- Index

- Notes

\section{Home}

Transactional Network Platform Overview

Getting started with Transactional Network Development

ExampleAgents

Agent Development

Established Topics

Working with the Actuator Agent

PlatformCommands 


\section{Configuring BACnet sMAP driver}

The device type specific configuration of the BACnet SMAP driver takes place in a CSV file specified in the sMAP configuration file. (See the register_config argument of the volttron.drivers.bacnet.BACnet in the sMAP config example here.) An example file can be found in the voltron repository at volttron/drivers/bacnet_example_config.csv

Most of the configuration file can be generated with the grab_bacnet_config.py utility. See AutoBacnetConfigGeneration.

If the target device is behind an IP to MS/TP router then Remote Station addressing will probably be needed for the driver to find the device.

If the device address is unknown then the bacnet_scan utility can be used to discover devices on a network.

Currently the driver provides no method to access array type properties even if the members of the array are of a supported type.

The CSV file should be comma delimited. An Excel spreadsheet converted to CSV should work fine, even with commas in the data.

The CSV file requires a header line with the following columns labeled in any order (without quotes):

\section{PNNL Point Name}


The name to used access the point. References to this point will use this name.

\section{Units}

Used for meta data when creating point information on the SMAP historian.

\section{BACnet Object Type}

A string representing what kind of BACnet standard object the point belongs to. Examples include:

- analoginput

- analogOutput

- analogValue

- binarylnput

- binaryOutput

- binaryValue

- multiStateValue

\section{Property}

A string representing the name of the property belonging to the object. Usually this will be "presentValue".

\section{Writable}


Either TRUE or FALSE. Determines if the point can be written to. If TRUE an actuation point will be created in addition to the normal point representing periodically scraped data. Only points labeled TRUE can be accessed through the ActuatorAgent. Incorrectly labeled points will cause an error to be returned if the user attempts to write to the point.

\section{Index}

Object ID of the BACnet object.

\section{Notes}

Additional notes for the point.

\section{Example}

Additional columns may be added at the user discretion as long as they are labeled differently.

The following is a simple example:

\begin{tabular}{|l|l|l|l|l|}
\hline \multicolumn{1}{|c|}{ Point Name } & PNNL Point Name & Units & $\begin{array}{c}\text { Unit } \\
\text { Details }\end{array}$ & $\begin{array}{r}\text { BACnet } \\
\text { Typ }\end{array}$ \\
\hline $\begin{array}{l}\text { Building1/FCB.Local } \\
\text { Application.DA1-P }\end{array}$ & SampleAnaloglnput & inchesOfWater & $\begin{array}{l}-0.20 \\
\text { to } 5.00\end{array}$ & analogInh \\
\hline $\begin{array}{l}\text { Building1/FCB.Local } \\
\text { Application.CLG-O }\end{array}$ & SampleAnalogOutput & percent & $\begin{array}{l}0.00 \text { to } \\
100.00\end{array}$ & analogOl \\
& & & & \\
\hline
\end{tabular}




\begin{tabular}{|c|c|c|c|c|}
\hline & & & $0.0)$ & \\
\hline Building1/Energy.OAT & SampleAnalogValue & days & $\begin{array}{l}\text { No } \\
\text { limits. } \\
\text { (default } \\
70.0 \text { ) }\end{array}$ & analog $\mathrm{Va}$ \\
\hline $\begin{array}{l}\text { Building1/FCB.Local } \\
\text { Application.LT-A }\end{array}$ & SampleBinarylnput & Enum & $0-1$ & binarylnp \\
\hline $\begin{array}{l}\text { Building1/FCB.Local } \\
\text { Application.GEF-C }\end{array}$ & SampleBinaryOutput & Enum & $\begin{array}{l}0-1 \\
\text { (default } \\
0 \text { ) }\end{array}$ & binaryOu \\
\hline $\begin{array}{l}\text { Building1/FCB.Local } \\
\text { Application.TUNING- } \\
\text { RESET }\end{array}$ & SampleBinaryValue & Enum & $\begin{array}{l}0-1 \\
\text { (default } \\
0 \text { ) }\end{array}$ & binaryVal \\
\hline $\begin{array}{l}\text { Building1/FCB.Local } \\
\text { Application.APP- } \\
\text { MODE }\end{array}$ & SampleMultiStateValue & State & $\begin{array}{l}\text { State } \\
\text { count: } \\
7 \\
\text { (default } \\
7 \text { ) }\end{array}$ & multiStat \\
\hline
\end{tabular}


Last edited by jhaack, April 23, 2014 


\section{VOLTTRON / volttron}

\begin{tabular}{l|l|l|l|}
\hline Star & 0 & $\square$ Fork & 1
\end{tabular}

Home Pages History

\section{ChangeLogs}

\section{$1 / 31 / 2014$}

The VOLTTRON(tm) 1.0 release includes the following features:

- Scheduler 2.0: The new ActuatorAgent scheduler allows applications to reserve devices ahead of time

- SchedulerExample: This simple agent provides an example of publishing a schedule request.

VOLTTRON v1.0 also includes features in a beta stage. These features are slated for release

Page History

Clone URL

Home

Transactional Network Platform Overview

Getting started with Transactional Network Development

ExampleAgents

Agent Development

Established Topics

Working with the Actuator Agent

PlatformCommands 
in v1.1 but are included in 1.0 for those who wish to begin investigating them. These features are:

PlatformStartup

PlatformServiceAgents

Working with the RTU Controller

Logging

Multi-building Messaging

BacnetDriver

\section{$11 / 7 / 2013$}

- Renamed Catalyst driver to Modbus driver to reflect the generic nature of the driver.

- Changed the configuration for the driver to fully take advantage of the Python struct module.

\section{$9 / 9 / 2013$}

- Catalyst registry file update for $372 \mathrm{~s}$

- catalystreg.csv.371 contains the points for the 371

\section{$9 / 4 / 2013$}

- Scheduling implemented

- Logging implemented

\section{$8 / 21 / 2013$}


- Added libevent-dev to required software

\section{$8 / 6 / 2013$}

WeatherAgent updated and back into the repository.

\section{$7 / 22 / 2017$}

The agent module was split into multiple pieces.

- The BaseAgent and PublishMixin classes and the periodic decorator remain in the agent package.

- The matching module was moved under the agent package and is now available as volttron.lite.agent.matching.

- The utility functions, like run_agent (which is deprecated) and the base agent ArgumentParser, were moved to volttron.lite.agent.utils.

All low-level messaging that is not agent-specific was moved to volttron.lite.messaging and includes the following new submodules:

- headers: contains common messaging headers, like CONTENT_TYPE, and values as constants

- topics: provides topic templates; see the module documentation for details

- utils: includes the Topic class and other messaging/topic utilities

The listener, control, archiver, and actuator agents were updated to use and demonstrate the changes above. Some of them also show how to use agent factories to perform dynamic matching. Using mercurial to show the diffs between revisions is a good technique for others to use to investigate how to migrate their agents. 


\section{$6 / 24 / 2013$}

- Initial version of ExampleControllerAgent committed. This agent monitors outdoor air temp and randomly sets the coolsuppy fan if temp has risen since the last reading. Wiki explanation for agent coming soon.

- Updates to ActuatorAgent

- ListenerAgent updated to reflect latest BaseAgent

- Use -config option instead of -config_path when starting agents

\section{$6 / 21 / 2013$}

- Updated ArchiverAgent checked in.

- ActuatorAgent for sending commands to the controller checked in.

\section{$6 / 19 / 2013$}

- Fixed a command line arg problem in ListenerAgent and updated wiki.

\section{Version 1.0}

This is the initial release of the Volttron Lite platform. The features contained in it are:

- Scripts for building the platform from scratch as well as updating

- A BaseAgent which expresses the basic functionality for an agent in the platform as well as hooks for adding functionality 
- Example agents which utilize the BaseAgent to illustrate more complex behavior

In addition, this wiki will be constantly updated with documentation for working with the platform and developing agents in it. We intend to document as much as possible but please submit TRAC tickets in cases where documentation does not exist yet or there is difficulty locating it. Also, this is a living document so feel free to add your own content to this wiki and even make changes to the documentation if you can improve on its clarity and usefulness.

Please subscribe to this page to receive notification when new changelogs are posted for future releases. 